\title{
In situ Switching of Site-Selectivity with Light in the Acetylation of Sugars with Azo-Peptide Catalysts
}

Dominik Niedek, ${ }^{\text {a }}$ Frederik R. Erb, ${ }^{a}$ Christopher Topp, ${ }^{\text {a }}$ Alexander Seitz, ${ }^{\text {a }}$ Raffael C. Wende, ${ }^{\text {a André K. }}$ Eckhardt, ${ }^{\mathrm{a}}$ Jonas Kind, ${ }^{\mathrm{b}}$ Dominik Herold, ${ }^{\mathrm{b}}$ Christina M. Thiele, ${ }^{\mathrm{b}}$ and Peter R. Schreiner*a

${ }^{a}$ Institute of Organic Chemistry, Justus Liebig University, Heinrich-Buff-Ring 17, 35392 Giessen (Germany) and ${ }^{b}$ ClemensSchöpf-Institut für Organische Chemie und Biochemie, Technische Universität Darmstadt, Alarich-Weiss-Str. 16, 64287 Darmstadt (Germany)

\section{Table of Contents}

$\begin{array}{lr}\text { Cartesian coordinates in } \AA \text { of optimized structures at the B3LYP-D3(BJ)/6-311G(d,p) level of theory } & \text { S2-S19 } \\ \text { Thermal, electronic and zero point vibrational energies of B3LYP-D3(BJ)/6-311G(d,p) optimized structures } & \text { S20-S22 } \\ \text { and corresponding DLPNO-CCSD(T)/def2-tzvpp single points } & \mathrm{S} 23-\mathrm{S} 25 \\ \text { UV/Vis data (in toluene) } & \mathrm{S} 26 \\ \text { Photochemistry equipment } & \mathrm{S} 27-\mathrm{S} 43 \\ \text { In situ irradiation NMR spectroscopy } & \mathrm{S} 44-\mathrm{S} 51 \\ \text { Determination of yields and selectivity via NMR } & \mathrm{S} 52 \\ \text { Additional results } & \mathrm{S} 53\end{array}$




\section{Cartesian coordinates in $A$ of optimized structures at the B3LYP-D3(BJ)/6-311G(d,p) level of theory $\left({ }^{1}\right.$ B3LYP-D3(BJ)/6-31G(d)) ${ }^{1}$}

\section{E-6}

\begin{tabular}{|c|c|c|c|}
\hline & $X$ & Y & Z \\
\hline 7 & 10.375910000 & -1.085558000 & 0.802782000 \\
\hline 6 & 11.784107000 & -1.415283000 & 0.725393000 \\
\hline 6 & 12.618368000 & -0.541608000 & 1.646467000 \\
\hline 8 & 13.785154000 & -0.738254000 & 1.870284000 \\
\hline 1 & 10.145867000 & -0.117726000 & 0.956734000 \\
\hline 1 & 11.911534000 & -2.450979000 & 1.044081000 \\
\hline 6 & 12.347847000 & -1.295327000 & -0.721587000 \\
\hline 6 & 12.201089000 & 0.100800000 & -1.267142000 \\
\hline 1 & 11.799968000 & -2.016342000 & -1.328757000 \\
\hline 1 & 13.396501000 & -1.596095000 & -0.694292000 \\
\hline 6 & 11.858556000 & 2.745374000 & -2.144700000 \\
\hline 6 & 11.025364000 & 0.491669000 & -1.915439000 \\
\hline 6 & 13.206413000 & 1.054065000 & -1.072464000 \\
\hline 6 & 13.037902000 & 2.365746000 & -1.507537000 \\
\hline 6 & 10.853473000 & 1.803363000 & -2.350252000 \\
\hline 1 & 10.243202000 & -0.239598000 & -2.085745000 \\
\hline 1 & 14.124619000 & 0.762184000 & -0.574523000 \\
\hline 1 & 13.829984000 & 3.090054000 & -1.354555000 \\
\hline 1 & 9.936067000 & 2.087933000 & -2.852646000 \\
\hline 1 & 11.727158000 & 3.765686000 & -2.485795000 \\
\hline 8 & 11.930093000 & 0.511902000 & 2.126231000 \\
\hline 6 & 12.694040000 & 1.453523000 & 2.903284000 \\
\hline 1 & 13.162815000 & 0.953337000 & 3.750522000 \\
\hline 1 & 11.979551000 & 2.200789000 & 3.238942000 \\
\hline 1 & 13.463707000 & 1.909615000 & 2.279815000 \\
\hline 6 & 9.430004000 & -1.939325000 & 0.316080000 \\
\hline 8 & 9.715167000 & -3.045968000 & -0.122995000 \\
\hline 6 & 8.019812000 & -1.433217000 & 0.339835000 \\
\hline 6 & 5.354565000 & -0.587053000 & 0.273009000 \\
\hline 6 & 7.089737000 & -2.076127000 & -0.488658000 \\
\hline 6 & 7.596732000 & -0.375658000 & 1.150717000 \\
\hline 6 & 6.273528000 & 0.043135000 & 1.116913000 \\
\hline 6 & 5.772146000 & -1.658202000 & -0.531470000 \\
\hline 1 & 7.434350000 & -2.908731000 & -1.088519000 \\
\hline 1 & 8.285554000 & 0.105041000 & 1.835224000 \\
\hline 1 & 5.922075000 & 0.854919000 & 1.741389000 \\
\hline 1 & 5.046802000 & -2.140009000 & -1.172195000 \\
\hline 7 & 4.036029000 & -0.076426000 & 0.313359000 \\
\hline 7 & 3.226323000 & -0.651958000 & -0.456426000 \\
\hline 6 & 1.910264000 & -0.157206000 & -0.428787000 \\
\hline 6 & -0.760400000 & 0.677293000 & -0.521461000 \\
\hline 6 & 0.998875000 & -0.795278000 & -1.278076000 \\
\hline 6 & 1.468140000 & 0.905054000 & 0.374192000 \\
\hline 6 & 0.150968000 & 1.324123000 & 0.333845000 \\
\hline 6 & -0.321520000 & -0.386469000 & -1.325202000 \\
\hline 1 & 1.353910000 & -1.614133000 & -1.891346000 \\
\hline 1 & 2.181070000 & 1.391179000 & 1.026010000 \\
\hline 1 & -0.193786000 & 2.139733000 & 0.949403000 \\
\hline 1 & -1.028103000 & -0.885993000 & -1.978700000 \\
\hline 7 & -2.107422000 & 1.038246000 & -0.625230000 \\
\hline 1 & -2.669571000 & 0.488261000 & -1.274633000 \\
\hline 7 & -4.929856000 & 0.677792000 & 0.160236000 \\
\hline 6 & -4.316252000 & 1.943370000 & -0.244080000 \\
\hline 6 & -2.814686000 & 1.945726000 & 0.122733000 \\
\hline 8 & -2.349657000 & 2.670713000 & 0.980737000 \\
\hline 1 & -5.465235000 & 0.637555000 & 1.014673000 \\
\hline 1 & -4.387211000 & 1.980113000 & -1.333859000 \\
\hline 6 & -5.050901000 & 3.127200000 & 0.373143000 \\
\hline 1 & -4.576532000 & 4.037972000 & -0.004307000 \\
\hline 1 & -4.872938000 & 3.130652000 & 1.453106000 \\
\hline 6 & -6.557592000 & 3.172446000 & 0.082767000 \\
\hline 6 & -8.673987000 & 4.551946000 & 0.434689000 \\
\hline 6 & -8.392827000 & 3.184199000 & -1.675141000 \\
\hline 6 & -8.991753000 & 4.454432000 & -1.061731000 \\
\hline 6 & -6.885272000 & 3.084647000 & -1.415470000 \\
\hline
\end{tabular}




\begin{tabular}{lllc}
\hline 6 & -7.167430000 & 4.444492000 & 0.693258000 \\
1 & -9.189737000 & 3.744570000 & 0.966621000 \\
1 & -8.876525000 & 2.300136000 & -1.245522000 \\
1 & -8.577892000 & 5.332325000 & -1.574044000 \\
1 & -6.364955000 & 3.896736000 & -1.942081000 \\
1 & -6.963110000 & 4.473082000 & 1.769014000 \\
1 & -7.038142000 & 2.311074000 & 0.563730000 \\
1 & -9.057853000 & 5.491087000 & 0.844987000 \\
1 & -8.587318000 & 3.155865000 & -2.751819000 \\
1 & -10.074201000 & 4.480465000 & -1.222063000 \\
1 & -6.513004000 & 2.144201000 & -1.829624000 \\
1 & -6.663959000 & 5.317984000 & 0.258363000 \\
7 & -6.623245000 & -1.293614000 & 0.981263000 \\
6 & -5.733262000 & -1.616629000 & -0.130589000 \\
6 & -4.929496000 & -0.411746000 & -0.627921000 \\
8 & -4.299380000 & -0.482341000 & -1.683290000 \\
1 & -6.617166000 & -1.944367000 & 1.752149000 \\
1 & -4.993413000 & -2.305652000 & 0.285909000 \\
6 & -6.414890000 & -2.316338000 & -1.335954000 \\
1 & -5.621512000 & -2.734757000 & -1.956986000 \\
1 & -6.911891000 & -1.558576000 & -1.938090000 \\
6 & -7.395098000 & -3.356131000 & -0.904224000 \\
6 & -7.301391000 & -4.406693000 & -0.018674000 \\
7 & -8.508404000 & -5.051736000 & 0.112318000 \\
6 & -9.330440000 & -4.406155000 & -0.683854000 \\
7 & -8.716799000 & -3.362464000 & -1.322107000 \\
1 & -6.429310000 & -4.734312000 & 0.527895000 \\
1 & -10.370716000 & -4.641663000 & -0.850909000 \\
6 & -9.329502000 & -2.470760000 & -2.294098000 \\
1 & -8.918774000 & -2.640763000 & -3.291797000 \\
1 & -10.400050000 & -2.670705000 & -2.316797000 \\
1 & -9.168319000 & -1.432490000 & -2.004117000 \\
6 & -7.859942000 & -0.728302000 & 0.760633000 \\
8 & -8.160387000 & -0.117885000 & -0.246861000 \\
8 & -8.630931000 & -0.932239000 & 1.838112000 \\
6 & -10.022871000 & -0.442561000 & 1.904131000 \\
6 & -10.476255000 & -0.939026000 & 3.273282000 \\
1 & -9.842949000 & -0.522992000 & 4.059135000 \\
1 & -11.508027000 & -0.632905000 & 3.457767000 \\
1 & -10.419727000 & -2.028051000 & 3.321158000 \\
6 & -10.848934000 & -1.083807000 & 0.790857000 \\
1 & -11.907454000 & -0.871908000 & 0.959430000 \\
1 & -10.707877000 & -2.166690000 & 0.795494000 \\
1 & -10.565747000 & -0.690306000 & -0.182878000 \\
6 & -10.035828000 & 1.082058000 & 1.835348000 \\
1 & -9.709429000 & 1.431857000 & 0.858614000 \\
1 & -11.049419000 & 1.447036000 & 2.017905000 \\
1 & -9.379496000 & 1.502329000 & 2.601009000 \\
& & & \\
\hline & & & \\
\hline & & & \\
1 & & & \\
1 & & & \\
1 & & & \\
1 & &
\end{tabular}




\begin{tabular}{|c|c|c|c|}
\hline & $X$ & $\mathrm{Y}$ & Z \\
\hline 7 & 6.444841000 & -1.295326000 & -0.727844000 \\
\hline 6 & 6.949248000 & -2.549611000 & -1.245000000 \\
\hline 6 & 7.361529000 & -3.496097000 & -0.130922000 \\
\hline 8 & 7.690347000 & -4.638866000 & -0.319921000 \\
\hline 1 & 6.705087000 & -1.040880000 & 0.209689000 \\
\hline 1 & 6.157988000 & -3.038492000 & -1.817373000 \\
\hline 6 & 8.162198000 & -2.340145000 & -2.198926000 \\
\hline 6 & 9.290702000 & -1.617554000 & -1.511307000 \\
\hline 1 & 7.793135000 & -1.770641000 & -3.052507000 \\
\hline 1 & 8.480305000 & -3.321994000 & -2.553124000 \\
\hline 6 & 11.292115000 & -0.259121000 & -0.086037000 \\
\hline 6 & 9.333095000 & -0.219903000 & -1.495666000 \\
\hline 6 & 10.272775000 & -2.324486000 & -0.809838000 \\
\hline 6 & 11.266193000 & -1.651817000 & -0.102826000 \\
\hline 6 & 10.324238000 & 0.455644000 & -0.788328000 \\
\hline 1 & 8.587213000 & 0.341213000 & -2.046980000 \\
\hline 1 & 10.254162000 & -3.408867000 & -0.818491000 \\
\hline 1 & 12.023483000 & -2.215427000 & 0.430283000 \\
\hline 1 & 10.342882000 & 1.539552000 & -0.790772000 \\
\hline 1 & 12.066302000 & 0.265252000 & 0.461957000 \\
\hline 8 & 7.376860000 & -2.902134000 & 1.077678000 \\
\hline 6 & 7.860540000 & -3.711578000 & 2.166581000 \\
\hline 1 & 7.268021000 & -4.622109000 & 2.252987000 \\
\hline 1 & 7.757753000 & -3.094451000 & 3.055402000 \\
\hline 1 & 8.905517000 & -3.973029000 & 1.997275000 \\
\hline 6 & 5.737200000 & -0.437860000 & -1.512747000 \\
\hline 8 & 5.364266000 & -0.741790000 & -2.639250000 \\
\hline 6 & 5.443108000 & 0.902333000 & -0.912670000 \\
\hline 6 & 4.801877000 & 3.423125000 & 0.108054000 \\
\hline 6 & 4.371321000 & 1.629664000 & -1.440821000 \\
\hline 6 & 6.224123000 & 1.476503000 & 0.096562000 \\
\hline 6 & 5.922237000 & 2.740832000 & 0.585253000 \\
\hline 6 & 4.038502000 & 2.872154000 & -0.928952000 \\
\hline 1 & 3.808911000 & 1.191761000 & -2.255442000 \\
\hline 1 & 7.104408000 & 0.966775000 & 0.470703000 \\
\hline 1 & 6.541569000 & 3.213137000 & 1.337833000 \\
\hline 1 & 3.194948000 & 3.422566000 & -1.324962000 \\
\hline 7 & 4.585358000 & 4.755491000 & 0.579663000 \\
\hline 7 & 3.476432000 & 5.169126000 & 0.969548000 \\
\hline 6 & 2.353252000 & 4.312772000 & 1.178041000 \\
\hline 6 & 0.021598000 & 2.840172000 & 1.663131000 \\
\hline 6 & 1.103336000 & 4.841906000 & 0.843350000 \\
\hline 6 & 2.419182000 & 3.066421000 & 1.810749000 \\
\hline 6 & 1.268045000 & 2.337369000 & 2.065991000 \\
\hline 6 & -0.047018000 & 4.101867000 & 1.051643000 \\
\hline 1 & 1.058198000 & 5.830642000 & 0.403637000 \\
\hline 1 & 3.374914000 & 2.667230000 & 2.121532000 \\
\hline 1 & 1.316913000 & 1.381346000 & 2.562168000 \\
\hline 1 & -1.012252000 & 4.498628000 & 0.756855000 \\
\hline 7 & -1.185038000 & 2.156287000 & 1.855511000 \\
\hline 1 & -2.024937000 & 2.621373000 & 1.511816000 \\
\hline 7 & -3.280270000 & 0.342681000 & 0.836184000 \\
\hline 6 & -2.863983000 & 0.458656000 & 2.234399000 \\
\hline 6 & -1.378203000 & 0.877845000 & 2.311010000 \\
\hline 8 & -0.507295000 & 0.134870000 & 2.722326000 \\
\hline 1 & -3.329121000 & -0.569633000 & 0.407722000 \\
\hline 1 & -3.457242000 & 1.271122000 & 2.661009000 \\
\hline 6 & -3.123668000 & -0.839054000 & 2.989997000 \\
\hline 1 & -2.841355000 & -0.670497000 & 4.033535000 \\
\hline 1 & -2.436033000 & -1.606582000 & 2.621267000 \\
\hline 6 & -4.570770000 & -1.346402000 & 2.923247000 \\
\hline 6 & -6.143312000 & -3.173510000 & 3.756332000 \\
\hline 6 & -7.029877000 & -0.844194000 & 3.319763000 \\
\hline 6 & -7.165273000 & -2.109776000 & 4.173427000 \\
\hline 6 & -5.601012000 & -0.289530000 & 3.349027000 \\
\hline 6 & -4.716600000 & -2.615206000 & 3.778529000 \\
\hline 1 & -6.378096000 & -3.522714000 & 2.744553000 \\
\hline 1 & -7.292522000 & -1.067321000 & 2.279909000 \\
\hline 1 & -7.004732000 & -1.852561000 & 5.228200000 \\
\hline
\end{tabular}




\begin{tabular}{rrrr}
\hline 1 & -5.358958000 & 0.055537000 & 4.363860000 \\
1 & -4.005489000 & -3.375132000 & 3.437007000 \\
1 & -4.804842000 & -1.617528000 & 1.886010000 \\
1 & -6.213649000 & -4.046835000 & 4.412117000 \\
1 & -7.732946000 & -0.079030000 & 3.663441000 \\
1 & -8.181172000 & -2.510707000 & 4.100695000 \\
1 & -5.542225000 & 0.581806000 & 2.691856000 \\
1 & -4.440001000 & -2.373936000 & 4.813391000 \\
7 & -4.470534000 & -0.337304000 & -1.519648000 \\
6 & -4.266992000 & 1.088221000 & -1.277008000 \\
6 & -3.732945000 & 1.390819000 & 0.125995000 \\
8 & -3.704364000 & 2.550447000 & 0.538991000 \\
1 & -4.125644000 & -0.686933000 & -2.400895000 \\
1 & -3.466869000 & 1.383680000 & -1.961147000 \\
6 & -5.499458000 & 1.985919000 & -1.564403000 \\
1 & -5.145036000 & 3.016942000 & -1.604000000 \\
1 & -6.176267000 & 1.923851000 & -0.714774000 \\
6 & -6.207676000 & 1.588871000 & -2.817020000 \\
6 & -5.761658000 & 1.352876000 & -4.098425000 \\
7 & -6.788238000 & 0.929147000 & -4.908790000 \\
6 & -7.849672000 & 0.907452000 & -4.134671000 \\
7 & -7.560727000 & 1.289213000 & -2.852505000 \\
1 & -4.758046000 & 1.468370000 & -4.480518000 \\
1 & -8.850510000 & 0.635489000 & -4.434510000 \\
6 & -8.510943000 & 1.409791000 & -1.758021000 \\
1 & -8.658089000 & 2.456701000 & -1.483469000 \\
1 & -9.464296000 & 0.995362000 & -2.083533000 \\
1 & -8.157991000 & 0.850412000 & -0.891660000 \\
6 & -5.615310000 & -0.974919000 & -1.095043000 \\
8 & -6.332282000 & -0.565645000 & -0.202516000 \\
8 & -5.767246000 & -2.109141000 & -1.792987000 \\
6 & -6.909218000 & -3.017556000 & -1.564288000 \\
6 & -6.661545000 & -4.114941000 & -2.594317000 \\
1 & -5.696931000 & -4.594290000 & -2.417027000 \\
1 & -7.445299000 & -4.872300000 & -2.527652000 \\
1 & -6.662186000 & -3.697506000 & -3.602881000 \\
6 & -8.217841000 & -2.284529000 & -1.852843000 \\
1 & -9.038742000 & -3.005382000 & -1.869670000 \\
1 & -8.166737000 & -1.795761000 & -2.828065000 \\
1 & -8.425198000 & -1.538240000 & -1.089120000 \\
6 & -6.851114000 & -3.568824000 & -0.142295000 \\
1 & -7.024616000 & -2.782490000 & 0.588785000 \\
1 & -7.616382000 & -4.338723000 & -0.017707000 \\
1 & -5.876286000 & -4.023474000 & 0.049149000 \\
\hline & & & \\
\hline
\end{tabular}


TS $Z-6 \rightarrow E-6$

\begin{tabular}{|c|c|c|c|}
\hline & $X$ & $Y$ & $Z$ \\
\hline 7 & 9.387198000 & 0.478468000 & 1.359319000 \\
\hline 6 & 10.682789000 & 0.869419000 & 1.865895000 \\
\hline 6 & 11.126280000 & 2.215490000 & 1.322956000 \\
\hline 8 & 12.097494000 & 2.807143000 & 1.720607000 \\
\hline 1 & 9.098193000 & 0.868212000 & 0.479363000 \\
\hline 1 & 10.623936000 & 0.952622000 & 2.952727000 \\
\hline 6 & 11.784184000 & -0.183035000 & 1.534763000 \\
\hline 6 & 11.925100000 & -0.388719000 & 0.049805000 \\
\hline 1 & 11.488691000 & -1.105637000 & 2.035553000 \\
\hline 1 & 12.721893000 & 0.161842000 & 1.973781000 \\
\hline 6 & 12.057790000 & -0.646276000 & -2.743424000 \\
\hline 6 & 11.099447000 & -1.294422000 & -0.624237000 \\
\hline 6 & 12.824155000 & 0.379704000 & -0.697081000 \\
\hline 6 & 12.891549000 & 0.252707000 & -2.082197000 \\
\hline 6 & 11.163368000 & -1.422233000 & -2.009353000 \\
\hline 1 & 10.404798000 & -1.904410000 & -0.058563000 \\
\hline 1 & 13.470530000 & 1.084759000 & -0.185748000 \\
\hline 1 & 13.598702000 & 0.852239000 & -2.644442000 \\
\hline 1 & 10.516443000 & -2.131156000 & -2.513616000 \\
\hline 1 & 12.110627000 & -0.747592000 & -3.821327000 \\
\hline 8 & 10.357978000 & 2.644539000 & 0.302082000 \\
\hline 6 & 10.795268000 & 3.855074000 & -0.341209000 \\
\hline 1 & 10.864057000 & 4.666506000 & 0.383278000 \\
\hline 1 & 10.043962000 & 4.071006000 & -1.096701000 \\
\hline 1 & 11.770858000 & 3.697969000 & -0.802715000 \\
\hline 6 & 8.673248000 & -0.527630000 & 1.951465000 \\
\hline 8 & 9.017029000 & -0.995710000 & 3.034071000 \\
\hline 6 & 7.479058000 & -1.011441000 & 1.216821000 \\
\hline 6 & 5.167114000 & -2.033363000 & -0.056986000 \\
\hline 6 & 6.555886000 & -1.804815000 & 1.914582000 \\
\hline 6 & 7.231855000 & -0.765434000 & -0.141782000 \\
\hline 6 & 6.112518000 & -1.257010000 & -0.783743000 \\
\hline 6 & 5.420122000 & -2.305834000 & 1.318550000 \\
\hline 1 & 6.761803000 & -2.019154000 & 2.956096000 \\
\hline 1 & 7.943131000 & -0.207700000 & -0.741228000 \\
\hline 1 & 5.944720000 & -1.070765000 & -1.836090000 \\
\hline 1 & 4.714056000 & -2.908987000 & 1.873800000 \\
\hline 7 & 4.083694000 & -2.502332000 & -0.647477000 \\
\hline 7 & 3.086067000 & -2.953091000 & -1.187158000 \\
\hline 6 & 1.896449000 & -2.152225000 & -1.144297000 \\
\hline 6 & -0.509918000 & -0.747468000 & -1.165189000 \\
\hline 6 & 0.776260000 & -2.703160000 & -1.767393000 \\
\hline 6 & 1.812744000 & -0.898353000 & -0.531613000 \\
\hline 6 & 0.622736000 & -0.193271000 & -0.537868000 \\
\hline 6 & -0.419843000 & -2.008709000 & -1.777677000 \\
\hline 1 & 0.869360000 & -3.676564000 & -2.233118000 \\
\hline 1 & 2.688756000 & -0.479855000 & -0.050563000 \\
\hline 1 & 0.544140000 & 0.774366000 & -0.068744000 \\
\hline 1 & -1.297184000 & -2.432052000 & -2.253506000 \\
\hline 7 & -1.746590000 & -0.103219000 & -1.218593000 \\
\hline 1 & -2.499859000 & -0.608509000 & -1.687287000 \\
\hline 7 & -4.449934000 & 0.377230000 & -0.139487000 \\
\hline 6 & -3.629395000 & 1.368924000 & -0.835948000 \\
\hline 6 & -2.129751000 & 1.070159000 & -0.613334000 \\
\hline 8 & -1.401888000 & 1.800716000 & 0.028709000 \\
\hline 1 & -4.881266000 & 0.622166000 & 0.739484000 \\
\hline 1 & -3.833677000 & 1.239778000 & -1.901879000 \\
\hline 6 & -3.990452000 & 2.782258000 & -0.395008000 \\
\hline 1 & -3.375031000 & 3.472943000 & -0.978953000 \\
\hline 1 & -3.677090000 & 2.919069000 & 0.644742000 \\
\hline 6 & -5.471445000 & 3.152198000 & -0.555055000 \\
\hline 6 & -7.146667000 & 5.055395000 & -0.277378000 \\
\hline 6 & -7.466496000 & 3.347732000 & -2.117283000 \\
\hline 6 & -7.669734000 & 4.807401000 & -1.696733000 \\
\hline 6 & -6.002565000 & 2.914472000 & -1.976706000 \\
\hline 6 & -5.685754000 & 4.615783000 & -0.137094000 \\
\hline 1 & -7.761761000 & 4.497090000 & 0.437156000 \\
\hline 1 & -8.082513000 & 2.691509000 & -1.492851000 \\
\hline 1 & -7.133983000 & 5.462223000 & -2.395696000 \\
\hline
\end{tabular}




\begin{tabular}{lccc}
\hline 1 & -5.381067000 & 3.476328000 & -2.687714000 \\
1 & -5.342151000 & 4.760348000 & 0.892827000 \\
1 & -6.070310000 & 2.524504000 & 0.117019000 \\
1 & -7.245652000 & 6.113082000 & -0.014703000 \\
1 & -7.799837000 & 3.201293000 & -3.149354000 \\
1 & -8.728064000 & 5.078284000 & -1.763677000 \\
1 & -5.915806000 & 1.857243000 & -2.240549000 \\
1 & -5.054759000 & 5.255959000 & -0.767618000 \\
7 & -6.435930000 & -0.943665000 & 1.178748000 \\
6 & -5.780916000 & -1.664951000 & 0.091096000 \\
6 & -4.789509000 & -0.800607000 & -0.692411000 \\
8 & -4.318856000 & -1.208165000 & -1.755203000 \\
1 & -6.488916000 & -1.433179000 & 2.059385000 \\
1 & -5.172710000 & -2.430526000 & 0.580667000 \\
6 & -6.741172000 & -2.376631000 & -0.898305000 \\
1 & -6.142303000 & -3.077446000 & -1.481810000 \\
1 & -7.123286000 & -1.637631000 & -1.599495000 \\
6 & -7.871136000 & -3.054662000 & -0.197093000 \\
6 & -7.910279000 & -3.925219000 & 0.869325000 \\
7 & -9.206595000 & -4.224740000 & 1.215168000 \\
6 & -9.952010000 & -3.548301000 & 0.370713000 \\
7 & -9.200770000 & -2.812749000 & -0.505565000 \\
1 & -7.075141000 & -4.354259000 & 1.403270000 \\
1 & -11.030902000 & -3.549909000 & 0.331791000 \\
6 & -9.709578000 & -1.984801000 & -1.587731000 \\
1 & -9.472831000 & -2.424532000 & -2.559122000 \\
1 & -10.792126000 & -1.916363000 & -1.488227000 \\
1 & -9.283790000 & -0.983343000 & -1.525842000 \\
6 & -7.531775000 & -0.142176000 & 0.940272000 \\
8 & -7.798884000 & 0.340531000 & -0.143044000 \\
8 & -8.200617000 & 0.033714000 & 2.087709000 \\
6 & -9.428807000 & 0.852438000 & 2.158042000 \\
6 & -9.823594000 & 0.718463000 & 3.625166000 \\
1 & -9.027187000 & 1.093466000 & 4.270763000 \\
1 & -10.731093000 & 1.293350000 & 3.820630000 \\
1 & -10.010555000 & -0.327475000 & 3.875144000 \\
6 & -10.500176000 & 0.254946000 & 1.248199000 \\
1 & -11.456032000 & 0.745431000 & 1.446868000 \\
1 & -10.610890000 & -0.812536000 & 1.449996000 \\
1 & -10.247270000 & 0.396772000 & 0.199783000 \\
6 & -9.102043000 & 2.299785000 & 1.801521000 \\
1 & -8.817456000 & 2.389740000 & 0.755675000 \\
1 & -9.978543000 & 2.926856000 & 1.981390000 \\
1 & -8.285086000 & 2.669538000 & 2.425599000 \\
\hline & & & \\
\hline
\end{tabular}




\section{E2-6*}

\begin{tabular}{|c|c|c|c|}
\hline & $x$ & $Y$ & Z \\
\hline 7 & -8.256732000 & -1.131939000 & -0.374348000 \\
\hline 6 & -9.566222000 & -0.620074000 & -0.018332000 \\
\hline 6 & -10.020319000 & 0.442219000 & -1.010997000 \\
\hline 8 & -11.111720000 & 0.959818000 & -0.976759000 \\
\hline 1 & -7.749301000 & -0.610163000 & -1.069165000 \\
\hline 1 & -10.299596000 & -1.433073000 & -0.065802000 \\
\hline 6 & -9.634733000 & -0.025847000 & 1.418573000 \\
\hline 6 & -8.576400000 & 1.017646000 & 1.674080000 \\
\hline 1 & -9.521662000 & -0.860019000 & 2.111697000 \\
\hline 1 & -10.638162000 & 0.392406000 & 1.540884000 \\
\hline 6 & -6.577508000 & 2.941794000 & 2.133035000 \\
\hline 6 & -7.392874000 & 0.674466000 & 2.341564000 \\
\hline 6 & -8.742032000 & 2.339134000 & 1.236876000 \\
\hline 6 & -7.750252000 & 3.294658000 & 1.463178000 \\
\hline 6 & -6.403450000 & 1.629612000 & 2.574970000 \\
\hline 1 & -7.262328000 & -0.347804000 & 2.687135000 \\
\hline 1 & -9.664544000 & 2.623993000 & 0.737885000 \\
\hline 1 & -7.903952000 & 4.318547000 & 1.132604000 \\
\hline 1 & -5.497796000 & 1.355152000 & 3.105664000 \\
\hline 1 & -5.809201000 & 3.686204000 & 2.323817000 \\
\hline 8 & -9.060150000 & 0.784983000 & -1.902336000 \\
\hline 6 & -9.427321000 & 1.829428000 & -2.824910000 \\
\hline 1 & -10.314999000 & 1.539572000 & -3.389998000 \\
\hline 1 & -8.569045000 & 1.951166000 & -3.485203000 \\
\hline 1 & -9.632535000 & 2.753342000 & -2.279352000 \\
\hline 6 & -7.634591000 & -2.126873000 & 0.316438000 \\
\hline 8 & -8.155842000 & -2.696093000 & 1.273592000 \\
\hline 6 & -6.241802000 & -2.459176000 & -0.138540000 \\
\hline 6 & -3.585794000 & -3.047225000 & -0.794114000 \\
\hline 6 & -5.447387000 & -3.208579000 & 0.737957000 \\
\hline 6 & -5.707409000 & -2.056724000 & -1.374735000 \\
\hline 6 & -4.387919000 & -2.335801000 & -1.701007000 \\
\hline 6 & -4.133930000 & -3.516504000 & 0.406667000 \\
\hline 1 & -5.887262000 & -3.539272000 & 1.672250000 \\
\hline 1 & -6.326373000 & -1.546974000 & -2.106667000 \\
\hline 1 & -3.963710000 & -2.017851000 & -2.646058000 \\
\hline 1 & -3.495191000 & -4.078476000 & 1.077141000 \\
\hline 7 & -2.215043000 & -3.317423000 & -0.987845000 \\
\hline 7 & -1.648174000 & -2.547973000 & -1.811334000 \\
\hline 6 & -0.260120000 & -2748285000 & -1.945075000 \\
\hline 6 & 2.531516000 & -2.817199000 & -2.135719000 \\
\hline 6 & 0.415572000 & -1.820156000 & -2.753202000 \\
\hline 6 & 0.471806000 & -3.743075000 & -1.277719000 \\
\hline 6 & 1.852892000 & -3.791723000 & -1.383584000 \\
\hline 6 & 1.801254000 & -1.840970000 & -2.838511000 \\
\hline 1 & -0.164097000 & -1.079194000 & -3.295529000 \\
\hline 1 & -0.057273000 & -4.452068000 & -0.652835000 \\
\hline 1 & 2.419553000 & -4.545420000 & -0.857240000 \\
\hline 1 & 2.331309000 & -1.099171000 & -3.429540000 \\
\hline 7 & 3.930834000 & -2.730771000 & -2.187086000 \\
\hline 1 & 4.277188000 & -1.982954000 & -2.786838000 \\
\hline 7 & 5.651252000 & -0.885432000 & -0.717128000 \\
\hline 6 & 6.075440000 & -2.203843000 & -1.201943000 \\
\hline 6 & 4.799013000 & -3.073680000 & -1.168404000 \\
\hline 8 & 4.553915000 & -3.866040000 & -0.273179000 \\
\hline 1 & 5681397000 & -0.708604000 & 0.278084000 \\
\hline 1 & 6.397968000 & -2.065542000 & -2.236751000 \\
\hline 6 & 7.210169000 & -2.759605000 & -0.349560000 \\
\hline 1 & 7.552061000 & -3.689874000 & -0.819674000 \\
\hline 1 & 6.807295000 & -3.049433000 & 0.628628000 \\
\hline 6 & 8.394586000 & -1.795606000 & -0.175819000 \\
\hline 6 & 10.696551000 & -1.523357000 & 0.879441000 \\
\hline 6 & 10.164407000 & -0.361280000 & -1.306489000 \\
\hline 6 & 11.257003000 & -1.022926000 & -0.457854000 \\
\hline 6 & 8.962834000 & -1.292830000 & -1.513448000 \\
\hline 6 & 9.498178000 & -2.456615000 & 0.666672000 \\
\hline 1 & 10.381184000 & -0.662063000 & 1.485704000 \\
\hline 1 & 9.816630000 & 0.556602000 & -0.811424000 \\
\hline 1 & 11.677612000 & -1.874191000 & -1.012110000 \\
\hline
\end{tabular}




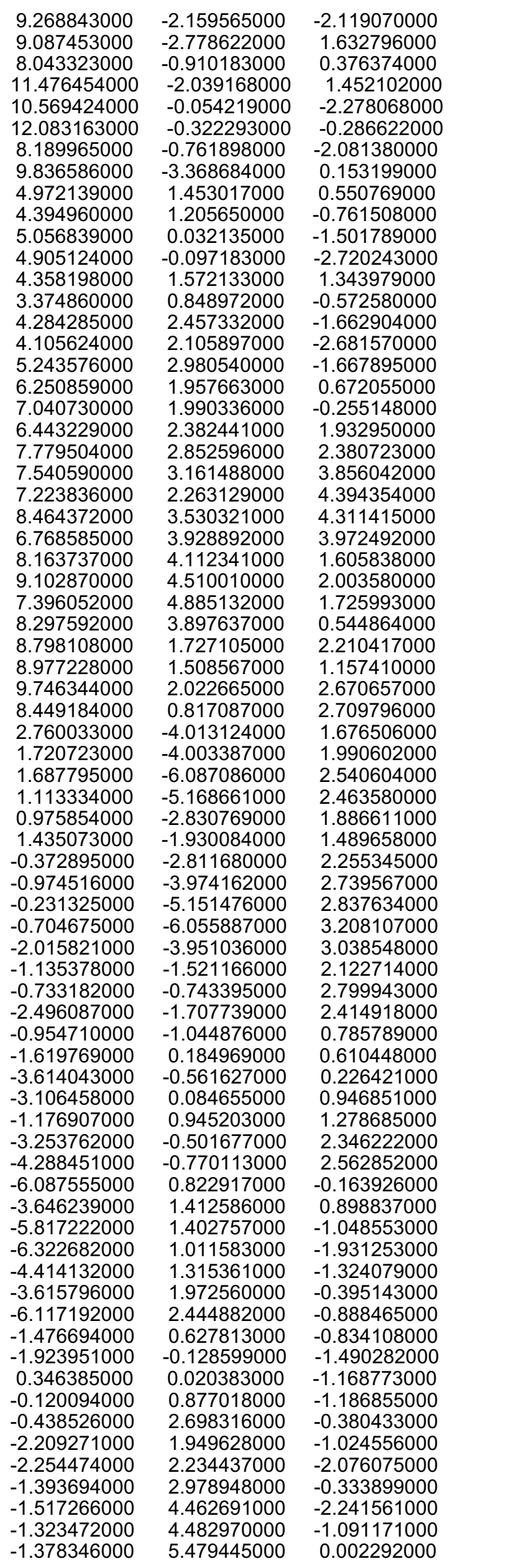




\begin{tabular}{lrrr}
\hline 1 & -2.434611000 & 5.628894000 & 0.258984000 \\
1 & -0.846097000 & 5.127591000 & 0.882998000 \\
1 & -0.962202000 & 6.419511000 & -0.365628000 \\
7 & 1.067412000 & 4.085415000 & -0.776391000 \\
6 & 1.812908000 & 3.219006000 & -1.551712000 \\
1 & 1.346000000 & 2.555110000 & -2.263224000 \\
6 & 3.145708000 & 3.319922000 & -1.233176000 \\
7 & 3.210245000 & 4.302495000 & -0.249356000 \\
6 & 1.942578000 & 4.728403000 & -0.013622000 \\
1 & 1.716701000 & 5.510000000 & 0.698173000 \\
1 & 5.207074000 & 4.935091000 & -0.346174000 \\
6 & 4.412401000 & 4.826720000 & 0.393913000 \\
1 & 4.757454000 & 4.164556000 & 1.190364000 \\
1 & 4.186563000 & 5.809184000 & 0.811898000 \\
1 & -2.900604000 & 0.223352000 & 3.096214000 \\
1 & -3.950717000 & 3.009909000 & -0.244656000 \\
\hline
\end{tabular}




\begin{tabular}{|c|c|c|c|}
\hline & $x$ & $Y$ & Z \\
\hline 7 & 0.965854000 & 2.598713000 & -1.840472000 \\
\hline 6 & 0.123808000 & 3.768092000 & -2.047322000 \\
\hline 6 & -0.638370000 & 4.207882000 & -0.793393000 \\
\hline 8 & -1.759703000 & 4.647454000 & -0.803180000 \\
\hline 1 & 1.934104000 & 2.630052000 & -2.135176000 \\
\hline 1 & 0.802016000 & 4.599147000 & -2.278444000 \\
\hline 6 & -0.842953000 & 3.589358000 & -3.248274000 \\
\hline 6 & -1.654493000 & 2.314554000 & -3.202190000 \\
\hline 1 & -0.226027000 & 3.596403000 & -4.153040000 \\
\hline 1 & -1.491852000 & 4.468084000 & -3.285055000 \\
\hline 6 & -3.073660000 & -0.109289000 & -3.058406000 \\
\hline 6 & -1.163927000 & 1.158404000 & -3.821917000 \\
\hline 6 & -2.870663000 & 2.243343000 & -2.511764000 \\
\hline 6 & -3.576268000 & 1.041933000 & -2.446378000 \\
\hline 6 & -1.859078000 & -0.047123000 & -3.742353000 \\
\hline 1 & -0.231665000 & 1.207679000 & -4.379348000 \\
\hline 1 & -3.254171000 & 3.125852000 & -2.013829000 \\
\hline 1 & -4.530255000 & 1.015250000 & -1.924060000 \\
\hline 1 & -1.466638000 & -0.935622000 & -4.228570000 \\
\hline 1 & -3.615463000 & -1.048337000 & -3.029115000 \\
\hline 8 & 0.120935000 & 4.189407000 & 0.342296000 \\
\hline 6 & -0.620796000 & 4.498293000 & 1.548455000 \\
\hline 1 & -0.976414000 & 5.528926000 & 1.516859000 \\
\hline 1 & -1.468671000 & 3.815526000 & 1.635885000 \\
\hline 1 & 0.086343000 & 4.357933000 & 2.366186000 \\
\hline 6 & 0.529548000 & 1.507657000 & -1.155430000 \\
\hline 8 & -0.483271000 & 1.560112000 & -0.450078000 \\
\hline 6 & 1.325344000 & 0.258787000 & -1.274238000 \\
\hline 6 & 2.607639000 & -2.217557000 & -1.457448000 \\
\hline 6 & 2.007255000 & -0.098839000 & -2.442931000 \\
\hline 6 & 1.324067000 & -0.620877000 & -0.183036000 \\
\hline 6 & 1.965345000 & -1.844698000 & -0.266572000 \\
\hline 6 & 2.667985000 & -1.323948000 & -2.529414000 \\
\hline 1 & 1.993845000 & 0.562834000 & -3.303111000 \\
\hline 1 & 0.815468000 & -0.326483000 & 0.726316000 \\
\hline 1 & 1.957443000 & -2.527124000 & 0.573743000 \\
\hline 1 & 3.189623000 & -1.612735000 & -3.436406000 \\
\hline 7 & 3.322118000 & -3.462494000 & -1.512890000 \\
\hline 7 & 2.724737000 & -4.551150000 & -1.343352000 \\
\hline 6 & 1293714000 & -4567071000 & -1.292357000 \\
\hline 6 & -1.487773000 & -4.448673000 & -1.026382000 \\
\hline 6 & 0.675343000 & -5.178050000 & -0.197530000 \\
\hline 6 & 0.506686000 & -4.019822000 & -2.313481000 \\
\hline 6 & -0.876190000 & -3.982504000 & -2.201925000 \\
\hline 6 & -0.702970000 & -5.087159000 & -0.049712000 \\
\hline 1 & 1.285671000 & -5.668524000 & 0.554681000 \\
\hline 1 & 0.981257000 & -3.617834000 & -3.201484000 \\
\hline 1 & -1.485028000 & -3.566755000 & -2.991843000 \\
\hline 1 & -1.184789000 & -5.504943000 & 0.829639000 \\
\hline 7 & -2.844649000 & -4.237485000 & -0.729836000 \\
\hline 1 & -3.052389000 & -4.253128000 & 0.266929000 \\
\hline 7 & -3.983698000 & -1.655468000 & 0.036301000 \\
\hline 6 & -4.749302000 & -2.745268000 & -0.577425000 \\
\hline 6 & -3.735179000 & -3.485993000 & -1.472150000 \\
\hline 8 & -3.656977000 & -3.314267000 & -2.678820000 \\
\hline 1 & -3857491000 & -0.792949000 & -0.481283000 \\
\hline 1 & -5.060222000 & -3.398786000 & 0.240526000 \\
\hline 6 & -5.966164000 & -2.217960000 & -1.327636000 \\
\hline 1 & -6.528890000 & -3.084897000 & -1.695812000 \\
\hline 1 & -5.637101000 & -1.675009000 & -2.221239000 \\
\hline 6 & -6.882471000 & -1.323569000 & -0.477612000 \\
\hline 6 & -9.051706000 & 0.019337000 & -0.513596000 \\
\hline 6 & -8.311200000 & -1.120169000 & 1.620412000 \\
\hline 6 & -9.510072000 & -0.677747000 & 0.773361000 \\
\hline 6 & -7.345879000 & -2.005275000 & 0.821041000 \\
\hline 6 & -8.089806000 & -0.867239000 & -1.314048000 \\
\hline 1 & -9.916206000 & 0.286408000 & -1.133087000 \\
\hline 1 & -8.651861000 & -1.655487000 & 2.514745000 \\
\hline 1 & -10.112172000 & -1.559575000 & 0.510626000 \\
\hline
\end{tabular}




\begin{tabular}{|c|c|c|c|}
\hline 1 & -7.842817000 & -2.954458000 & 0.567141000 \\
\hline 1 & -8.630553000 & -1.757791000 & -1.667532000 \\
\hline 1 & -6.316302000 & -0.426137000 & -0.186104000 \\
\hline 1 & -8.548472000 & 0.961297000 & -0.255386000 \\
\hline 1 & -7.765993000 & -0.235242000 & 1.976742000 \\
\hline 1 & -10.162513000 & -0.014576000 & 1.354048000 \\
\hline 1 & -6.482662000 & -2.253110000 & 1.449885000 \\
\hline 1 & -7.741391000 & -0.339787000 & -2.212222000 \\
\hline 7 & -2.708296000 & 0.584254000 & 0.951600000 \\
\hline 6 & -2.273338000 & -0.687299000 & 1.479050000 \\
\hline 6 & -3.244793000 & -1.831039000 & 1.144933000 \\
\hline 8 & -3.240717000 & -2.889715000 & 1.790191000 \\
\hline 1 & -2.082300000 & 1.088597000 & 0.331389000 \\
\hline 1 & -1.376499000 & -0.935865000 & 0.900659000 \\
\hline 6 & -1.894792000 & -0.671582000 & 2.995869000 \\
\hline 1 & -2.499216000 & -1.412268000 & 3.523542000 \\
\hline 1 & -2.162906000 & 0.303655000 & 3.404518000 \\
\hline 6 & -3.824538000 & 1.195732000 & 1.451389000 \\
\hline 8 & -4.560523000 & 0.671227000 & 2.275060000 \\
\hline 8 & -3.964189000 & 2.414564000 & 0.890181000 \\
\hline 6 & -5.117972000 & 3.272524000 & 1.229465000 \\
\hline 6 & -6.420941000 & 2.557573000 & 0.873708000 \\
\hline 1 & -6.591443000 & 1.695735000 & 1.518355000 \\
\hline 1 & -6.394353000 & 2.222106000 & -0.168777000 \\
\hline 1 & -7.260349000 & 3.252328000 & 0.982196000 \\
\hline 6 & -4.909341000 & 4.491558000 & 0.334729000 \\
\hline 1 & -3.927013000 & 4.938547000 & 0.503276000 \\
\hline 1 & -4.976187000 & 4.211916000 & -0.721247000 \\
\hline 1 & -5.682415000 & 5.237961000 & 0.542265000 \\
\hline 6 & -5.046568000 & 3.654240000 & 2.708064000 \\
\hline 1 & -5.152981000 & 2.774890000 & 3.345015000 \\
\hline 1 & -4.091469000 & 4.144708000 & 2.927806000 \\
\hline 1 & -5.850215000 & 4.359101000 & 2.944724000 \\
\hline 6 & -0.436054000 & -0.909929000 & 3.223664000 \\
\hline 6 & 0.621856000 & -0.031715000 & 3.319361000 \\
\hline 7 & 1.817633000 & -0.726462000 & 3.389574000 \\
\hline 6 & 1.486630000 & -2.007936000 & 3.350228000 \\
\hline 7 & 0.144568000 & -2.173571000 & 3.249773000 \\
\hline 1 & 0.589352000 & 1.048677000 & 3.308977000 \\
\hline 1 & 2.173271000 & -2.842374000 & 3.383962000 \\
\hline 6 & -0.542007000 & -3.463860000 & 3.304036000 \\
\hline 1 & -1.470105000 & -3.408525000 & 2.734307000 \\
\hline 1 & -0.768772000 & -3.725826000 & 4.341849000 \\
\hline 1 & 0.111954000 & -4.222798000 & 2.870765000 \\
\hline 6 & 4.160155000 & -0.604385000 & 2.936455000 \\
\hline 8 & 4.214998000 & -1.335862000 & 2.044724000 \\
\hline 6 & 4.621825000 & 1.243889000 & 0.243588000 \\
\hline 6 & 3.234397000 & 3.078998000 & 1.247269000 \\
\hline 6 & 4.292056000 & 1.995503000 & 1.515735000 \\
\hline 6 & 3.575249000 & 3.909058000 & -0.007273000 \\
\hline 6 & 4.999096000 & 2.234451000 & -0.860795000 \\
\hline 1 & 3.737344000 & 0.679136000 & -0.080242000 \\
\hline 1 & 5.194528000 & 2.413453000 & 1.962528000 \\
\hline 1 & 5.862580000 & 2.823526000 & -0.535363000 \\
\hline 1 & 3.186667000 & 3.770484000 & 2.097753000 \\
\hline 1 & 2.695052000 & 4.486599000 & -0.318841000 \\
\hline 8 & 4.625806000 & 4.743372000 & 0.366837000 \\
\hline 6 & 4.960729000 & 5.731179000 & -0.609507000 \\
\hline 1 & 5.325879000 & 5.269539000 & -1.532809000 \\
\hline 1 & 5.745211000 & 6.346898000 & -0.168599000 \\
\hline 1 & 4.087852000 & 6.357465000 & -0.838856000 \\
\hline 8 & 2.025063000 & 2.352441000 & 1.111926000 \\
\hline 8 & 3.690495000 & 1.131804000 & 2.534390000 \\
\hline 1 & 1.298078000 & 2.935532000 & 0.805457000 \\
\hline 1 & 2.742846000 & 1.033721000 & 2.278064000 \\
\hline 6 & 5.378942000 & 1.438313000 & -2.104759000 \\
\hline 1 & 4.492079000 & 0.921650000 & -2.497289000 \\
\hline 1 & 5.790882000 & 2.083288000 & -2.883881000 \\
\hline 8 & 5.712261000 & 0.372126000 & 0.470399000 \\
\hline 8 & 6.394431000 & 0.503392000 & -1.756837000 \\
\hline 6 & 5.989665000 & -0.375954000 & -0.724521000 \\
\hline 1 & 5.061932000 & -0.885219000 & -1.022609000 \\
\hline
\end{tabular}




\begin{tabular}{lccc}
\hline 6 & 7.077259000 & -1.360214000 & -0.430581000 \\
6 & 9.086441000 & -3.202098000 & 0.154331000 \\
6 & 8.348247000 & -0.910464000 & -0.058424000 \\
6 & 6.811292000 & -2.728569000 & -0.512133000 \\
6 & 7.819110000 & -3.649197000 & -0.219295000 \\
6 & 9.350893000 & -1.831531000 & 0.233431000 \\
1 & 8.542915000 & 0.156118000 & -0.008135000 \\
1 & 5.824846000 & -3.073217000 & -0.814310000 \\
1 & 7.614221000 & -4.713401000 & -0.289396000 \\
1 & 10.340005000 & -1.484267000 & 0.517572000 \\
1 & 9.871235000 & -3.918809000 & 0.378593000 \\
8 & 3.889944000 & 3.098962000 & -1.134090000 \\
6 & 4.657080000 & -0.414685000 & 4.311433000 \\
1 & 5.637314000 & 0.071308000 & 4.237387000 \\
1 & 3.973181000 & 0.207220000 & 4.882378000 \\
1 & 4.772603000 & -1.400425000 & 4.769517000 \\
\hline
\end{tabular}




\begin{tabular}{|c|c|c|c|}
\hline & $X$ & $\mathrm{Y}$ & Z \\
\hline 7 & 7.932499000 & 1.520855000 & 0.466353000 \\
\hline 6 & 9.263100000 & 1.010379000 & 0.749958000 \\
\hline 6 & 9.819486000 & 0.279837000 & -0.469006000 \\
\hline 8 & 10.521809000 & -0.706719000 & -0.419948000 \\
\hline 1 & 7.346369000 & 0.989496000 & -0.163614000 \\
\hline 1 & 9.911364000 & 1.883842000 & 0.899918000 \\
\hline 6 & 9.350687000 & 0.155404000 & 2.037149000 \\
\hline 6 & 8.270574000 & -0.890846000 & 2.167003000 \\
\hline 1 & 9.284719000 & 0.851983000 & 2.875055000 \\
\hline 1 & 10.340616000 & -0.307686000 & 2.055293000 \\
\hline 6 & 6.244145000 & -2.821476000 & 2.452621000 \\
\hline 6 & 7.083691000 & -0.588749000 & 2.848378000 \\
\hline 6 & 8.424666000 & -2.173602000 & 1.623464000 \\
\hline 6 & 7.419340000 & -3.131502000 & 1.764922000 \\
\hline 6 & 6.079572000 & -1.545687000 & 2.993910000 \\
\hline 1 & 6.959848000 & 0.400579000 & 3.280111000 \\
\hline 1 & 9.342857000 & -2.416621000 & 1.097653000 \\
\hline 1 & 7.559245000 & -4.124356000 & 1.346271000 \\
\hline 1 & 5.171393000 & -1.295309000 & 3.535102000 \\
\hline 1 & 5.465656000 & -3.570503000 & 2.568753000 \\
\hline 8 & 9.478854000 & 0.895821000 & -1.616515000 \\
\hline 6 & 10.069090000 & 0.343213000 & -2.806352000 \\
\hline 1 & 11.150272000 & 0.501935000 & -2.796791000 \\
\hline 1 & 9.609149000 & 0.879539000 & -3.635678000 \\
\hline 1 & 9.869487000 & -0.728271000 & -2.872761000 \\
\hline 6 & 7.355311000 & 2.440356000 & 1.290303000 \\
\hline 8 & 7.939262000 & 2.927864000 & 2.255652000 \\
\hline 6 & 5.935942000 & 2.794777000 & 0.963186000 \\
\hline 6 & 3.235070000 & 3.334771000 & 0.509781000 \\
\hline 6 & 5.115759000 & 3.203832000 & 2.021071000 \\
\hline 6 & 5.415789000 & 2.738110000 & -0.338844000 \\
\hline 6 & 4.075008000 & 3.014811000 & -0.569982000 \\
\hline 6 & 3.773643000 & 3.480099000 & 1.796935000 \\
\hline 1 & 5.553219000 & 3.292314000 & 3.009376000 \\
\hline 1 & 6.062941000 & 2.488203000 & -1.173786000 \\
\hline 1 & 3.657754000 & 2.972832000 & -1.569704000 \\
\hline 1 & 3.112470000 & 3.774682000 & 2.605621000 \\
\hline 7 & 1.838428000 & 3.499357000 & 0.406673000 \\
\hline 7 & 1.312035000 & 2.919068000 & -0.587006000 \\
\hline 6 & -0.086792000 & 3.089245000 & -0.692898000 \\
\hline 6 & -2.856586000 & 3.344962000 & -1.080034000 \\
\hline 6 & -0.705659000 & 2.493759000 & -1.803669000 \\
\hline 6 & -0.873026000 & 3.816016000 & 0.220988000 \\
\hline 6 & -2.239301000 & 3.949565000 & 0.033839000 \\
\hline 6 & -2.073961000 & 2.621303000 & -1.998861000 \\
\hline 1 & -0.092224000 & 1.933678000 & -2.502703000 \\
\hline 1 & -0.385049000 & 4.283071000 & 1.068504000 \\
\hline 1 & -2.843563000 & 4.509916000 & 0.732834000 \\
\hline 1 & -2.555088000 & 2.149305000 & -2.851527000 \\
\hline 7 & -4.241170000 & 3.382042000 & -1.292505000 \\
\hline 1 & -4.553308000 & 2.886389000 & -2.125667000 \\
\hline 7 & -5.989216000 & 1.261577000 & -0.574418000 \\
\hline 6 & -6.463280000 & 2.650005000 & -0.615395000 \\
\hline 6 & -5.204132000 & 3.491053000 & -0.297622000 \\
\hline 8 & -5.031674000 & 4.049488000 & 0.770222000 \\
\hline 1 & -5.846805000 & 0.863836000 & 0.343074000 \\
\hline 1 & -6.794748000 & 2.834712000 & -1.639717000 \\
\hline 6 & -7.590810000 & 2.864224000 & 0.390930000 \\
\hline 1 & -8.014183000 & 3.861168000 & 0.219055000 \\
\hline 1 & -7.156352000 & 2.894393000 & 1.397595000 \\
\hline 6 & -8.694293000 & 1.798502000 & 0.325217000 \\
\hline 6 & -10.836431000 & 0.964017000 & 1.408432000 \\
\hline 6 & -10.469364000 & 0.643235000 & -1.074794000 \\
\hline 6 & -11.506061000 & 0.877023000 & 0.031096000 \\
\hline 6 & -9.369848000 & 1.713897000 & -1.053064000 \\
\hline 6 & -9.739465000 & 2.035852000 & 1.426913000 \\
\hline 1 & -10.390902000 & -0.011132000 & 1.655871000 \\
\hline 1 & -10.005878000 & -0.344346000 & -0.934597000 \\
\hline 1 & -12.042011000 & 1.816022000 & -0.168484000 \\
\hline
\end{tabular}




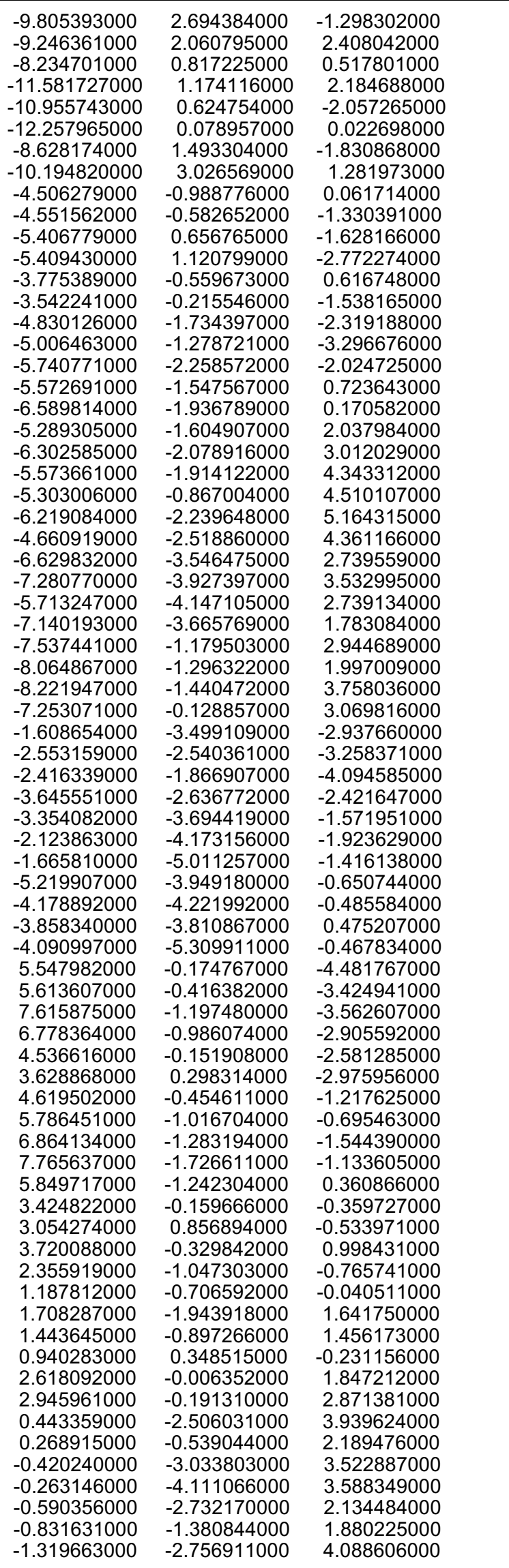




\begin{tabular}{rrrr}
\hline 6 & 0.029264000 & -1.569850000 & -0.472706000 \\
1 & 0.277582000 & -2.628898000 & -0.360748000 \\
1 & -1.211943000 & 0.707669000 & 0.352169000 \\
6 & -1.207080000 & -1.277067000 & 0.389766000 \\
1 & -1.982090000 & -2.017849000 & 0.177135000 \\
8 & -1.800779000 & -0.024265000 & 0.093610000 \\
1 & 2.342588000 & 1.049942000 & 1.745632000 \\
1 & -1.657030000 & -0.994892000 & 2.492632000 \\
8 & 0.244299000 & -1.706041000 & -4.284142000 \\
6 & 0.517934000 & -2.291204000 & -3.332134000 \\
6 & 1.456039000 & -3.236336000 & -2.723314000 \\
1 & 2.198378000 & -2.650698000 & -2.164177000 \\
1 & 0.933238000 & -3.916482000 & -2.054801000 \\
1 & 1.921661000 & -3.792905000 & -3.541783000 \\
1 & -1.188058000 & -1.365651000 & -2.069401000 \\
8 & -0.227042000 & -1.288359000 & -1.873269000 \\
\hline
\end{tabular}




\begin{tabular}{|c|c|c|c|}
\hline & $x$ & $Y$ & Z \\
\hline 7 & -1.615882000 & 0.131957000 & 2.775935000 \\
\hline 6 & -1.527324000 & 1.522658000 & 3.213669000 \\
\hline 6 & -1.501232000 & 2.518311000 & 2.051481000 \\
\hline 8 & -0.858442000 & 3.539541000 & 2.064025000 \\
\hline 1 & -2.021169000 & -0.515735000 & 3.436700000 \\
\hline 1 & -2.471408000 & 1.736756000 & 3.730813000 \\
\hline 6 & -0.367707000 & 1.763921000 & 4.211913000 \\
\hline 6 & 0.973341000 & 1.236623000 & 3.757654000 \\
\hline 1 & -0.648750000 & 1.277544000 & 5.153122000 \\
\hline 1 & -0.321756000 & 2.839026000 & 4.403508000 \\
\hline 6 & 3.440997000 & 0.198268000 & 2.898772000 \\
\hline 6 & 1.368754000 & -0.059150000 & 4.113158000 \\
\hline 6 & 1.826887000 & 2.005670000 & 2.957751000 \\
\hline 6 & 3.054451000 & 1.492330000 & 2.540242000 \\
\hline 6 & 2.586436000 & -0.581027000 & 3.679086000 \\
\hline 1 & 0.723511000 & -0.659209000 & 4.751198000 \\
\hline 1 & 1.521480000 & 3.000548000 & 2.656405000 \\
\hline 1 & 3712591000 & 2117270000 & 1.940994000 \\
\hline 1 & 2.882015000 & -1.585890000 & 3.966505000 \\
\hline 1 & 4.400326000 & -0.209469000 & 2.598927000 \\
\hline 8 & -2.401993000 & 2.220917000 & 1.085060000 \\
\hline 6 & -2.497696000 & 3.204327000 & 0.023203000 \\
\hline 1 & -2.797358000 & 4.167832000 & 0.437782000 \\
\hline 1 & -1.529091000 & 3.298041000 & -0.473426000 \\
\hline 1 & -3.267199000 & 2.820065000 & -0.640173000 \\
\hline 6 & -0.797932000 & -0.374147000 & 1.806703000 \\
\hline 8 & -0.242667000 & 0.376663000 & 0.998158000 \\
\hline 6 & -0.677639000 & -1.853164000 & 1.708994000 \\
\hline 6 & -0.214368000 & -4.599652000 & 1.413290000 \\
\hline 6 & -0.847591000 & -2.724115000 & 2.794699000 \\
\hline 6 & -0.320585000 & -2.384187000 & 0.460632000 \\
\hline 6 & -0.096076000 & -3.741446000 & 0.308664000 \\
\hline 6 & -0.642987000 & -4.093091000 & 2.644148000 \\
\hline 1 & -1.093019000 & -2.342277000 & 3.781695000 \\
\hline 1 & -0.211736000 & -1.707252000 & -0.377797000 \\
\hline 1 & 0.199274000 & -4.143261000 & -0.652460000 \\
\hline 1 & -0.761317000 & -4.769494000 & 3.484444000 \\
\hline 7 & -0.044597000 & -6.013785000 & 1.253070000 \\
\hline 7 & 1.022573000 & -6.492753000 & 0.803827000 \\
\hline 6 & 2164759000 & -5.648866000 & 0.630091000 \\
\hline 6 & 4.285107000 & -3.889851000 & 0.152664000 \\
\hline 6 & 2.807031000 & -5.657352000 & -0.612016000 \\
\hline 6 & 2.673760000 & -4.855626000 & 1.665990000 \\
\hline 6 & 3.745090000 & -4.001317000 & 1.444650000 \\
\hline 6 & 3.834985000 & -4.756615000 & -0.858909000 \\
\hline 1 & 2.457180000 & -6.331351000 & -1.388026000 \\
\hline 1 & 2.225666000 & -4.906290000 & 2.652105000 \\
\hline 1 & 4.146275000 & -3.396005000 & 2.244799000 \\
\hline 1 & 4.295099000 & -4.711314000 & -1.841966000 \\
\hline 7 & 5.201520000 & -2.889673000 & -0.214594000 \\
\hline 1 & 5.171572000 & -2.658537000 & -1.206023000 \\
\hline 7 & 4.482610000 & -0.069236000 & -0.493258000 \\
\hline 6 & 5.839851000 & -0.551331000 & -0.220271000 \\
\hline 6 & 5.636040000 & -1.850683000 & 0.584599000 \\
\hline 8 & 5.716799000 & -1.901691000 & 1.802485000 \\
\hline 1 & 4.007699000 & 0.484160000 & 0.210509000 \\
\hline 1 & 6.284380000 & -0.784945000 & -1.190301000 \\
\hline 6 & 6.666176000 & 0.503224000 & 0.506138000 \\
\hline 1 & 7.689703000 & 0.118362000 & 0.596024000 \\
\hline 1 & 6.296392000 & 0.610469000 & 1.532669000 \\
\hline 6 & 6.681667000 & 1.871953000 & -0.192865000 \\
\hline 6 & 7.619777000 & 4.239590000 & -0.061005000 \\
\hline 6 & 7.195323000 & 3.175156000 & -2.317372000 \\
\hline 6 & 8.077513000 & 4.144596000 & -1.521818000 \\
\hline 6 & 7.136670000 & 1.790260000 & -1.659736000 \\
\hline 6 & 7.563034000 & 2.854874000 & 0.595915000 \\
\hline 1 & 8.289135000 & 4.895931000 & 0.507938000 \\
\hline 1 & 7.561492000 & 3.082810000 & -3.346885000 \\
\hline 1 & 9.118777000 & 3.792255000 & -1.550887000 \\
\hline
\end{tabular}




\begin{tabular}{|c|c|c|}
\hline 8.132458000 & 1.322021000 & -1.698576000 \\
\hline 8.581445000 & 2.443424000 & 0.658966000 \\
\hline 5.655728000 & 2.270089000 & -0.196650000 \\
\hline 6.622977000 & 4.700251000 & -0.023674000 \\
\hline 6.174582000 & 3.576138000 & -2.384968000 \\
\hline 8.069551000 & 5.137055000 & -1.988423000 \\
\hline 6.457541000 & 1.149197000 & -2.233890000 \\
\hline 7.197185000 & 2.936174000 & 1.628295000 \\
\hline 1.988142000 & 1.028743000 & -0.685838000 \\
\hline 2.256318000 & -0.147182000 & -1.481893000 \\
\hline 3.745547000 & -0.522063000 & -1.520797000 \\
\hline 4.183582000 & -1.300881000 & -2.380349000 \\
\hline 1.281593000 & 0.969056000 & 0.041906000 \\
\hline 1.808570000 & -0.973691000 & -0.918798000 \\
\hline 1.632334000 & -0.130431000 & -2.912837000 \\
\hline 2.403894000 & -0.392910000 & -3.639874000 \\
\hline 1.316235000 & 0.888027000 & -3.141052000 \\
\hline 2.413440000 & 2.258261000 & -1.115263000 \\
\hline 3.129193000 & 2.408686000 & -2.095773000 \\
\hline 1.948350000 & 3.216374000 & -0.291315000 \\
\hline 2.256505000 & 4.639229000 & -0.541140000 \\
\hline 3.768250000 & 4.861514000 & -0.508548000 \\
\hline 4.260277000 & 4.383676000 & -1.355191000 \\
\hline 4.188332000 & 4.459317000 & 0.419811000 \\
\hline 3.979728000 & 5.935662000 & -0.535293000 \\
\hline 1.581061000 & 5.343774000 & 0.632611000 \\
\hline 0.521279000 & 5.085315000 & 0.688530000 \\
\hline 2.050272000 & 5.054831000 & 1.578397000 \\
\hline 1.680742000 & 6.427811000 & 0.518777000 \\
\hline 1.632266000 & 5.063150000 & -1.870844000 \\
\hline 2.087449000 & 4.528055000 & -2.705746000 \\
\hline 0.553952000 & 4.867553000 & -1.862122000 \\
\hline 1.780348000 & 6.137610000 & -2.020177000 \\
\hline 0.461271000 & -1.050083000 & -3.018291000 \\
\hline-0.881979000 & -0.834590000 & -2.817126000 \\
\hline-1.571344000 & -2.034937000 & -2.872040000 \\
\hline-0.658559000 & -2.964169000 & -3.115522000 \\
\hline 0.582418000 & -2.425068000 & -3.207540000 \\
\hline-1.383663000 & 0.093517000 & -2.588632000 \\
\hline-0.851590000 & -4.021748000 & -3.231871000 \\
\hline 1.799600000 & -3.158511000 & -3.554530000 \\
\hline 2.664711000 & -2.670420000 & -3.103326000 \\
\hline 1.928504000 & -3.183632000 & -4.640691000 \\
\hline 1.716389000 & -4.177682000 & -3.172901000 \\
\hline-3.763485000 & -3.140059000 & -2.552572000 \\
\hline-3.483232000 & -4.262838000 & -2.631110000 \\
\hline-4.569391000 & -2.138201000 & -3.272838000 \\
\hline-5.546969000 & -2.083634000 & -2.780606000 \\
\hline-4.079043000 & -1.168286000 & -3.241199000 \\
\hline-4.675745000 & -2.480957000 & -4.304941000 \\
\hline-5.183654000 & 0.136326000 & -0.602347000 \\
\hline-4.108051000 & -2.022431000 & 0.192849000 \\
\hline-4.045866000 & -0.482369000 & 0.189552000 \\
\hline-5.521813000 & -2.623454000 & 0.208142000 \\
\hline-6.512965000 & -0.533322000 & -0.272346000 \\
\hline-4.983669000 & 0.003986000 & -1.675715000 \\
\hline-4.124611000 & -0.140158000 & 1.226891000 \\
\hline-6.719635000 & -0.434341000 & 0.797975000 \\
\hline-3.557567000 & -2.416482000 & 1.048543000 \\
\hline-5.509235000 & -3.646065000 & -0.194745000 \\
\hline-5.900290000 & -2.607912000 & 1.547386000 \\
\hline-7.132379000 & -3.288367000 & 1.809194000 \\
\hline-7.968918000 & -2.805666000 & 1.294472000 \\
\hline-7.283035000 & -3.241248000 & 2.887907000 \\
\hline-7.066219000 & -4.336981000 & 1.491436000 \\
\hline-3.361084000 & -2.534353000 & -0.958635000 \\
\hline-2.789924000 & -0.136213000 & -0.366108000 \\
\hline-2.590976000 & -1.935160000 & -1.143272000 \\
\hline-2.404118000 & 0.601654000 & 0.148659000 \\
\hline-7.612253000 & 0.172346000 & -1.056901000 \\
\hline-7.467424000 & 0.015544000 & -2.138173000 \\
\hline-8.607649000 & -0.181258000 & -0.781908000 \\
\hline
\end{tabular}




\begin{tabular}{lrrr}
\hline 8 & -5.257480000 & 1.517904000 & -0.308640000 \\
8 & -7.557963000 & 1.559684000 & -0.736087000 \\
6 & -6.312515000 & 2.145947000 & -1.047262000 \\
1 & -6.104782000 & 2.004533000 & -2.122546000 \\
6 & -6.310152000 & 3.596835000 & -0.670948000 \\
6 & -6.158158000 & 6.277888000 & 0.088008000 \\
6 & -6.709469000 & 3.982822000 & 0.612431000 \\
6 & -5.836833000 & 4.552556000 & -1.570924000 \\
6 & -5.759800000 & 5.893807000 & -1.192416000 \\
6 & -6.635901000 & 5.321491000 & 0.988617000 \\
1 & -7.079648000 & 3.231613000 & 1.302106000 \\
1 & -5.530173000 & 4.250168000 & -2.569367000 \\
1 & -5.395786000 & 6.635640000 & -1.896802000 \\
1 & -6.951824000 & 5.622345000 & 1.983084000 \\
1 & -6.102470000 & 7.321328000 & 0.383683000 \\
8 & -6.428121000 & -1.920028000 & -0.631660000 \\
\hline
\end{tabular}


Thermal, electronic and zero-point vibrational energies of B3LYP-D3(BJ)/6-311G(d,p) optimized structures Z-6/E-6 and corresponding DLPNO-CCSD(T)/def2-tzvpp single point energies

Table S1. Electronic and zero-point vibrational energies of Z-6/E-6 in atomic units (au).

\begin{tabular}{ccccc}
\hline Structure & $E_{\mathrm{e}} / \mathrm{au}(\mathrm{B} 3 \mathrm{LYP})$ & $E_{\mathrm{e}} / \mathrm{au}($ Single Point) & ZPVE / au & $\mathrm{V}_{\mathrm{i}} \mathrm{cm}^{-1}$ \\
\hline$E-6$ & -2674.82191 & -2669.45603 & 0.941865 & $/$ \\
Z-6 & -2674.80154 & -2669.43866 & 0.941309 & $/$ \\
TS $Z$ Z-6 $\rightarrow E-6$ & -2674.76460 & -2669.39114 & 0.939495 & -369.6624 \\
\hline
\end{tabular}

Table S2. Thermochemistry of E-6 and Z-6.

\begin{tabular}{ccc}
\hline Structure & Thermal Correction $\Delta \boldsymbol{H} /$ au & Thermal Correction $\Delta \boldsymbol{G} /$ au \\
\hline$E-6$ & 1.000317 & 0.837290 \\
$Z-6$ & 0.999665 & 0.836888 \\
TS $Z-6 \rightarrow E-6$ & 0.997855 & 0.835385 \\
\hline
\end{tabular}

Table S3. Computed thermodynamic parameters at $298 \mathrm{~K}$ of the thermal conversion from Z-6 to $E-6$.

\begin{tabular}{cccccc}
\hline Structure & $\Delta \boldsymbol{E}_{Z-E} / \mathrm{kcal} \mathrm{mol}^{-1}$ & $\Delta \boldsymbol{H}_{Z-E} / \mathrm{kcal} \mathrm{mol}^{-1}$ & $\Delta \boldsymbol{G}_{Z-E} / \mathrm{kcal} \mathrm{mol}^{-1}$ & $\boldsymbol{k}_{Z-E} / \mathbf{s}^{-1}$ & $\Delta \boldsymbol{H}^{+}{ }_{Z-E} / \mathrm{kcal} \mathrm{mol}^{-1}$ \\
\hline $\mathbf{6}$ (DFT) & 12.4 & 12.4 & 12.5 & $3.1 \cdot 10^{-4}$ & 22.1 \\
\hline $\mathbf{6}$ (CC-Single Point) & 10.5 & 10.5 & 10.6 & $4.1 \cdot 10^{-9}$ & 28.7 \\
\hline
\end{tabular}

Table S4. Computed half-lives of Z-6 at relevant temperatures based on computed data

\begin{tabular}{cccc}
\hline Structure & $\boldsymbol{t}_{1 / 2}(273 \mathrm{~K})$ & $\boldsymbol{t}_{1 / 2}(298 \mathrm{~K})$ & $\boldsymbol{t}_{1 / 2}(\mathbf{3 2 3} \mathrm{K})$ \\
\hline Z-6 (DFT) & $0.89 \mathrm{~d}$ & $0.63 \mathrm{~h}$ & $1.9 \mathrm{~min}$ \\
\hline Z-6 (CCSD(T)-Single Point) & $185 \times 10^{3} \mathrm{~d}$ & $1950 \mathrm{~d}$ & $41 \mathrm{~d}$ \\
\hline
\end{tabular}

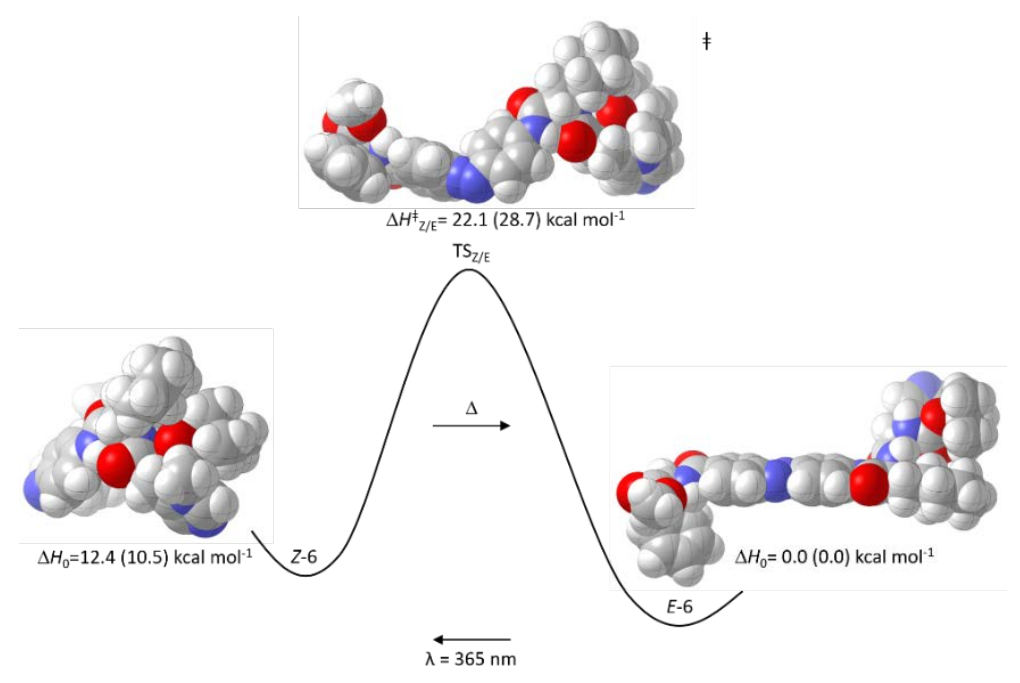


The obtained results for the ZIE isomerization are comparable to those of Schweighauser et al. who also used Grimme's ${ }^{2}$ dispersioncorrected B3LYP-D3(BJ) level. ${ }^{3}$ Vibrational frequencies were computed to ensure the nature of the localized stationary point. All reported transition state structures display exactly one imaginary vibrational frequency while minima exhibit only real vibrational modes. To improve the quality of the electronic energy single point energies were computed at the DLPNO-CCSD(T)/def2-tzvpp level of theory on top of the optimized structures (in parentheses). ${ }^{4}$ Note: In the isomerization process itself there is maybe a conical intersection to the triplet $T_{1}$ state and therefore the computed values for the singlet $S_{0}$ state TS are too high in case of probably more accurate coupledcluster energies. ${ }^{5}$ Therefore, the half-lives obtained from DFT computations should be used as a comparison value as this method is accepted in the literature. ${ }^{3}$

Table S5. Electronic and zero-point vibrational energies of acetylation transition structures at B3LYP-D3(BJ)/6-31G(d) level of theory.

\begin{tabular}{|c|c|c|c|c|}
\hline Structure & $\begin{array}{c}E_{\mathrm{e}} / \mathrm{au}\left(\mathrm{B} 3 \mathrm{~L} Y \mathrm{P}_{-}\right. \\
\mathrm{D} 3(\mathrm{BJ}) / 6-31 \mathrm{G}(\mathrm{d}))\end{array}$ & ZPVE / au & $\mathrm{v}_{\mathrm{i}} \mathrm{Cm}^{-1}$ & $\Delta \boldsymbol{E} / \mathrm{kcal} \mathrm{mol}^{-1}$ \\
\hline$E 2-6^{*}$ & -3822.8920409 & 1.3161771 & -81.6 & 0.0 \\
\hline E3-6" & -3822.8857916 & 1.3160337 & -96.5 & 3.8 \\
\hline$Z 3-6^{\ddagger}$ & -3822.8982705 & 1.3171181 & -90.1 & 0.0 \\
\hline$Z 2-6^{\mp}$ & -3822.8864047 & 1.3163237 & -128.3 & 6.9 \\
\hline
\end{tabular}

Table S6. Electronic and zero-point vibrational single point energies of acetylation transition structures at B3LYP-D3(BJ)/cc-pVTZ/I B3LYP-D3(BJ)/6-31G(d) level of theory.

\begin{tabular}{cccc}
\hline Structure & $\begin{array}{c}\boldsymbol{E}_{\mathrm{e}} / \mathrm{au}(\mathrm{B} 3 \mathrm{LYP}-\mathrm{D} 3(\mathrm{BJ}) / \mathrm{cc}-\mathrm{pVTZ//} \\
\text { B3LYP-D3(BJ)/6-31G(d)) }\end{array}$ & ZPVE / au & $\Delta E / \mathrm{kcal} \mathrm{mol}^{-1}$ \\
\hline E2-6 & -3824.2533800 & 1.3161771 & $\mathbf{0 . 0}$ \\
$E 3-\mathbf{6}^{\ddagger}$ & -3824.2496180 & 1.3160337 & $\mathbf{2 . 3}$ \\
\hline Z3-6 $^{\ddagger}$ & -3824.2531184 & 1.3171181 & $\mathbf{0 . 0}$ \\
Z2-6 $^{\ddagger}$ & -3824.2428299 & 1.3163237 & $\mathbf{6 . 0}$ \\
\hline
\end{tabular}




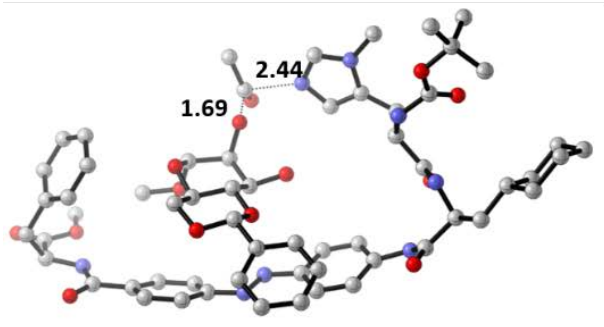

E2-6”

inside catalytic event

(2-acetylation)

$\Delta E 0.0(0.0) \mathrm{kcal} \mathrm{mol}^{-1}$

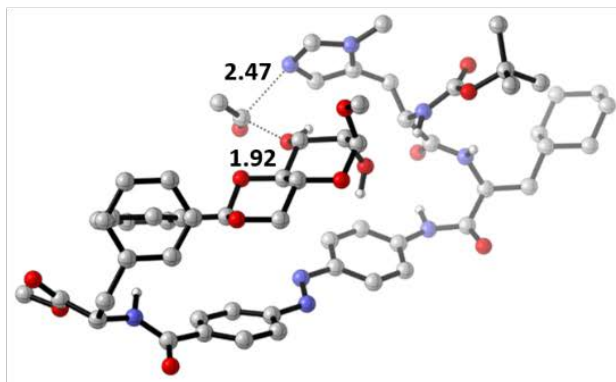

E3-6‡

inside catalytic event

(3-acetylation)

$\Delta E 3.8$ (2.3) kcal mol-1

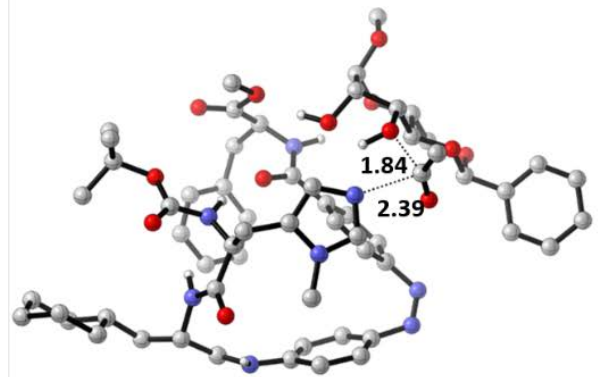

23-6”

outside catalytic event

(3-acetylation)

$\Delta E 0.0(0.0) \mathrm{kcal} \mathrm{mol}^{-1}$

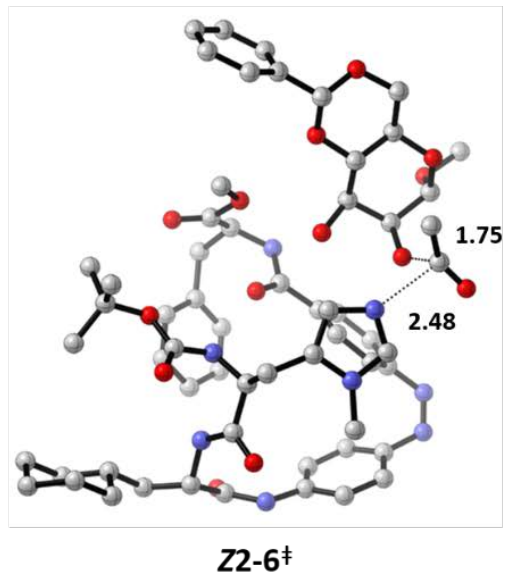

outside catalytic event

(2-acetylation)

$\Delta E 6.9$ (6.0) kcal mol-1 


\section{UV/Vis data}

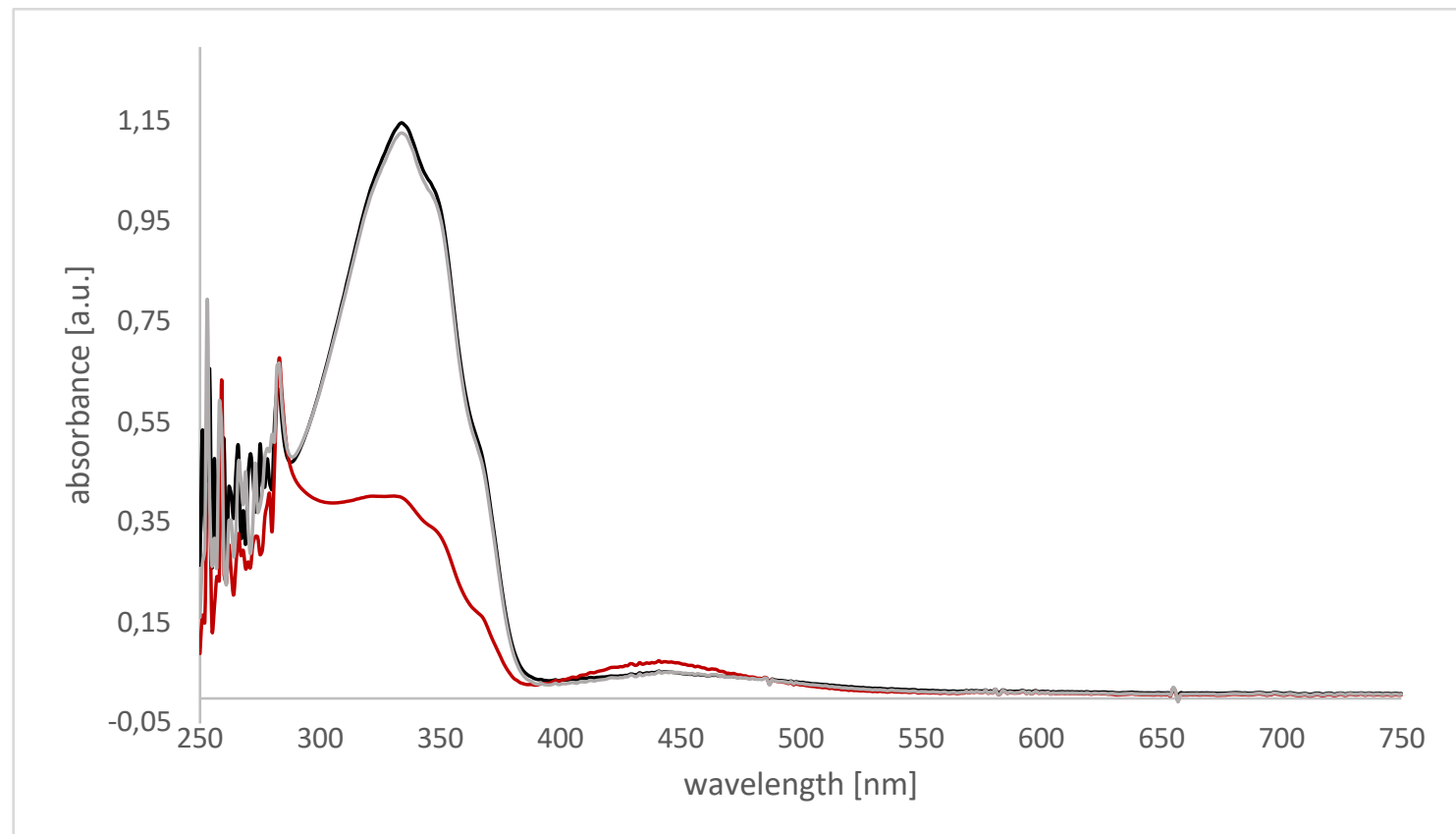

Figure S1 UV/Vis of 4 in toluene $\left(c=10^{-5} \mathrm{M}\right)$ : daylight (black), 1 min irradiation $\lambda=365 \mathrm{~nm}$ (red, $\left.Z-4\right)$ and $1 \mathrm{~min}$ irradiation of $Z-4 \lambda=448 \mathrm{~nm}(\mathrm{grey}, E-4)$.

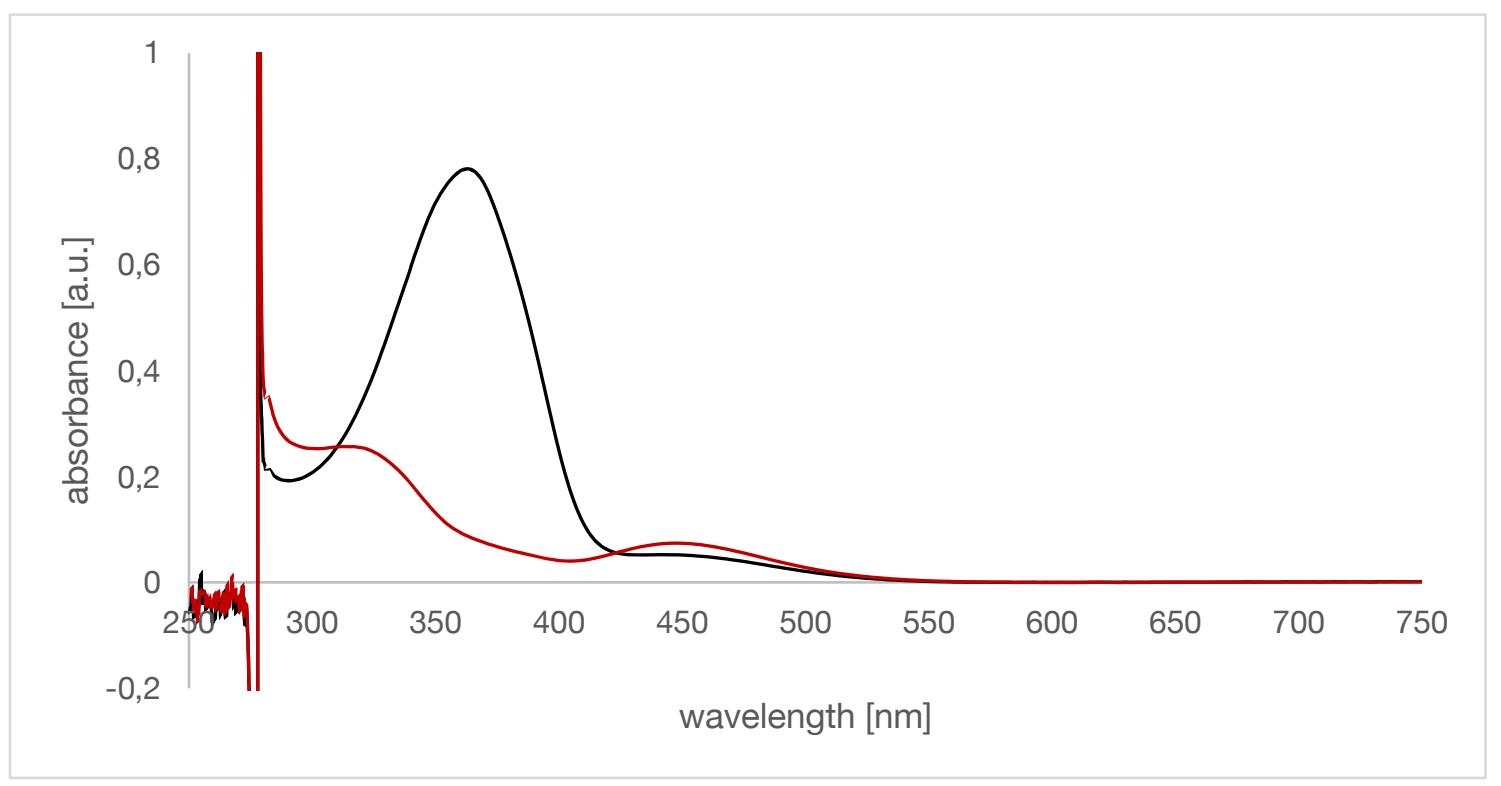

Figure S2 UV/Vis of 5 in toluene $\left(c=10^{-5} \mathrm{M}\right)$ : daylight (black, $\left.E-\mathbf{5}\right), 1 \mathrm{~min}$ irradiation $\lambda=365 \mathrm{~nm}(\mathrm{red}, \mathrm{Z}-\mathbf{5})$. 


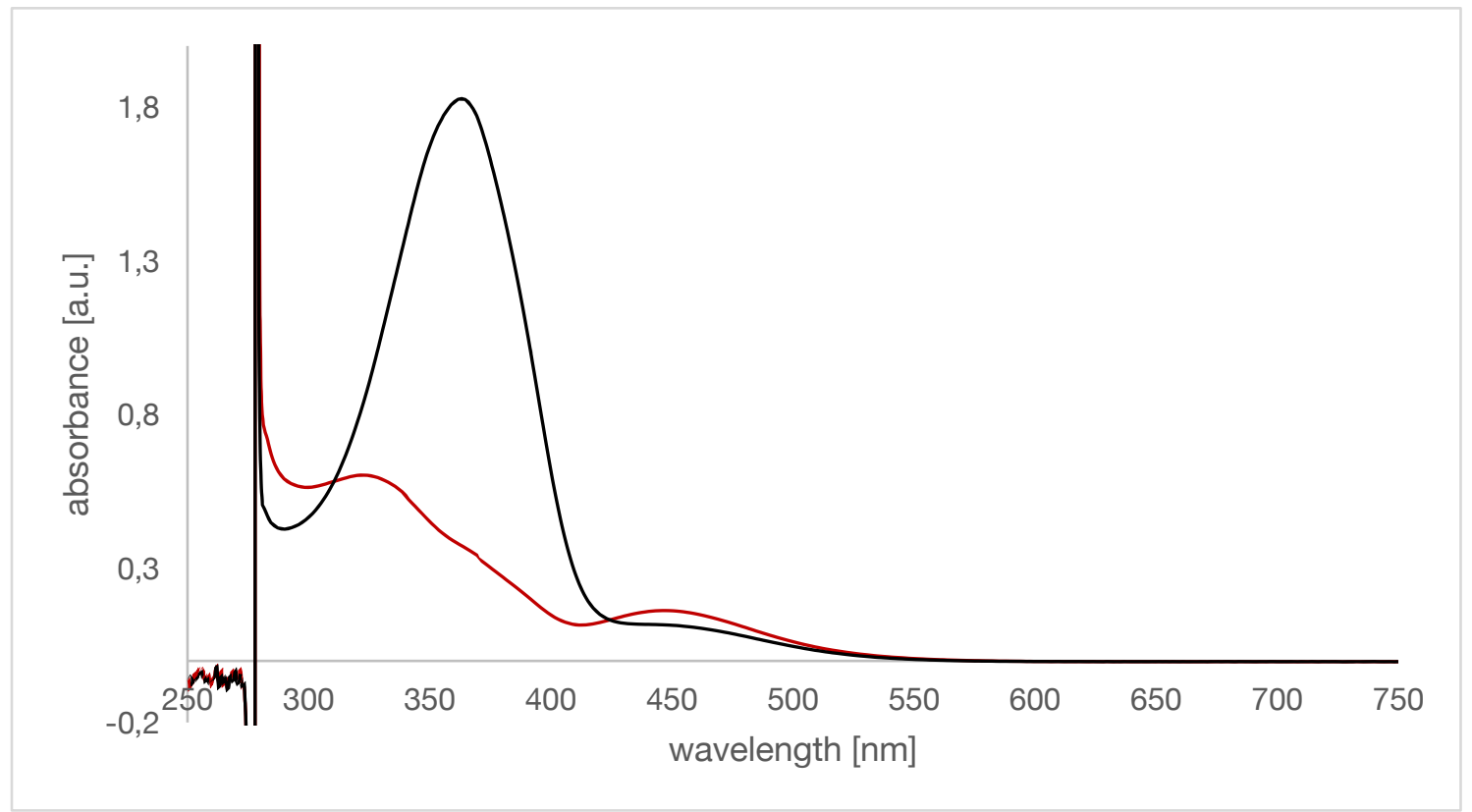

Figure S3 UV/Vis of 6 in toluene $\left(c=10^{-5} \mathrm{M}\right)$ : daylight (black, $\left.E-6\right)$, 1 min irradiation $\lambda=365 \mathrm{~nm}$ (red, Z-6).

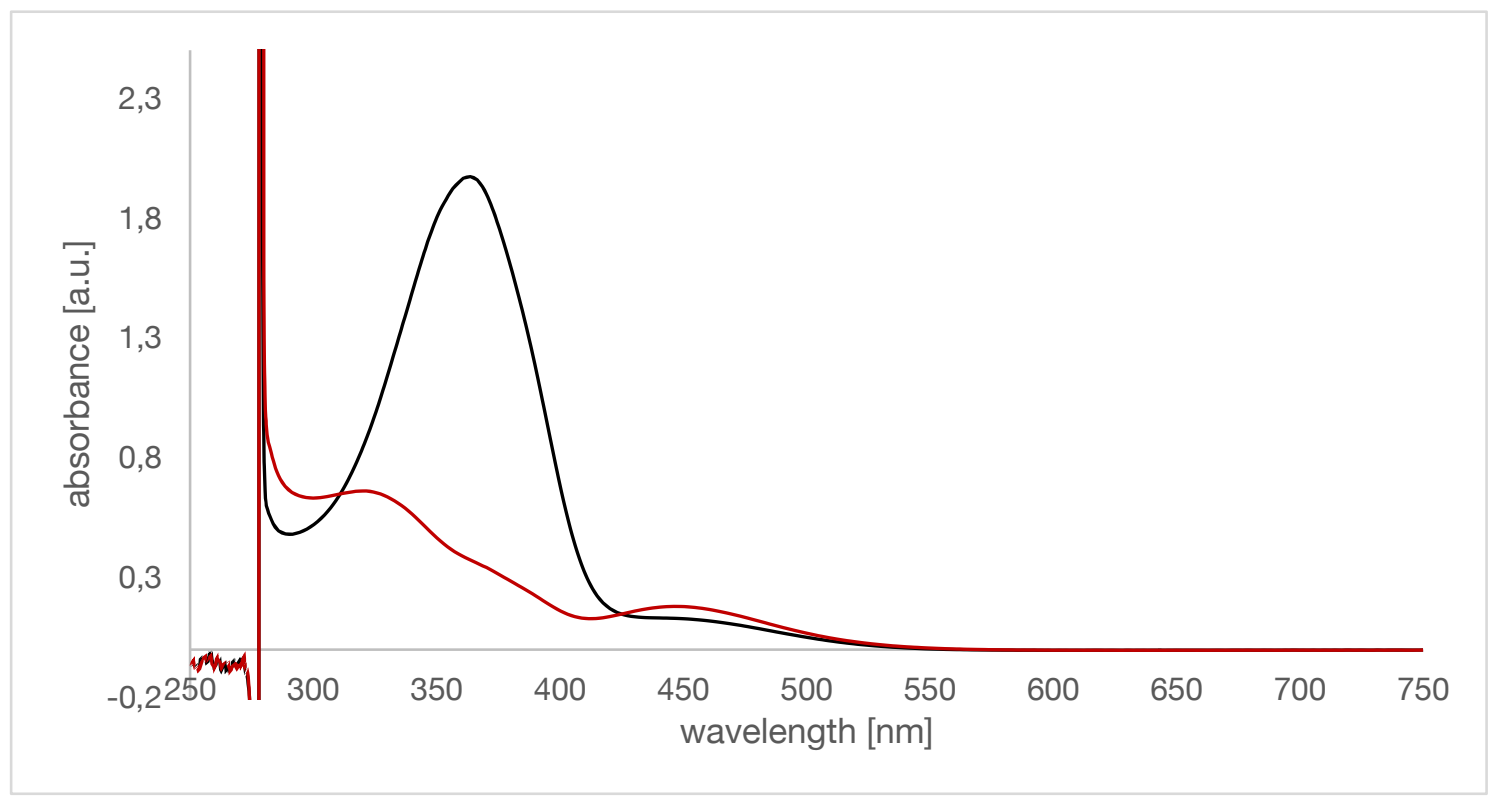

Figure S4 UV/Vis of 7 in toluene $\left(c=10^{-5} \mathrm{M}\right)$ : daylight (black, E-7), 1 min irradiation $\lambda=365 \mathrm{~nm}$ (red, Z-7).

As expected for amino azobenzene type photochromes, all four catalysts show bathochromic shifts of the $\pi \rightarrow \pi^{*}$ transition compared to azobenzene, whereas the $n \rightarrow \pi^{*}$ transitions remain unaffected by the substitution. ${ }^{6}$ By irradiation of the $\pi \rightarrow \pi^{*}$ transition of the $E$-isomers all four catalysts can be isomerized effectively into the Z-isomer (red trace), which show hypsochromically shifted $\pi \rightarrow \pi^{*}$ transitions and isochromatic $n \rightarrow \pi^{*}$ transitions. ${ }^{7}$ The back-isomerization of the $Z$-isomer into the $E$-isomer can be realized effectively by irradiation with $448 \mathrm{~nm}$. Note that the concentration of the corresponding $E$-isomer is slightly higher (grey trace) compared to the spectra obtained from the non-equilibrated daylight samples (black trace). Azobenzenes of the amino azobenzene type typically show a shorter half-life of the thermal $Z \rightarrow E$ isomerization than observed for the unsubstituted azobenzene. 


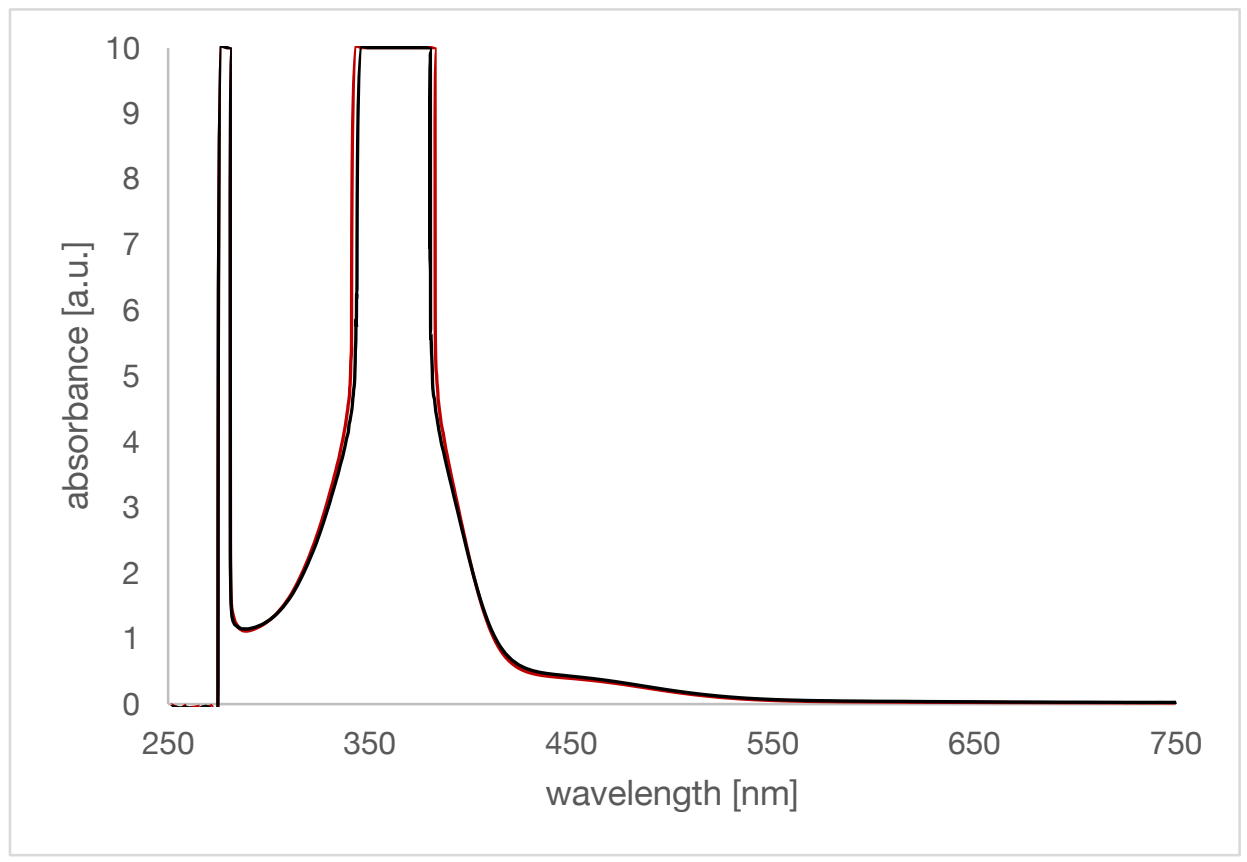

Figure S5 UV/Vis of 6 in toluene $\left(\mathrm{c}=2.0010^{-4} \mathrm{M}\right)$ : daylight, r.t. (black, E-6), daylight, $0{ }^{\circ} \mathrm{C}$ (red, E-6).

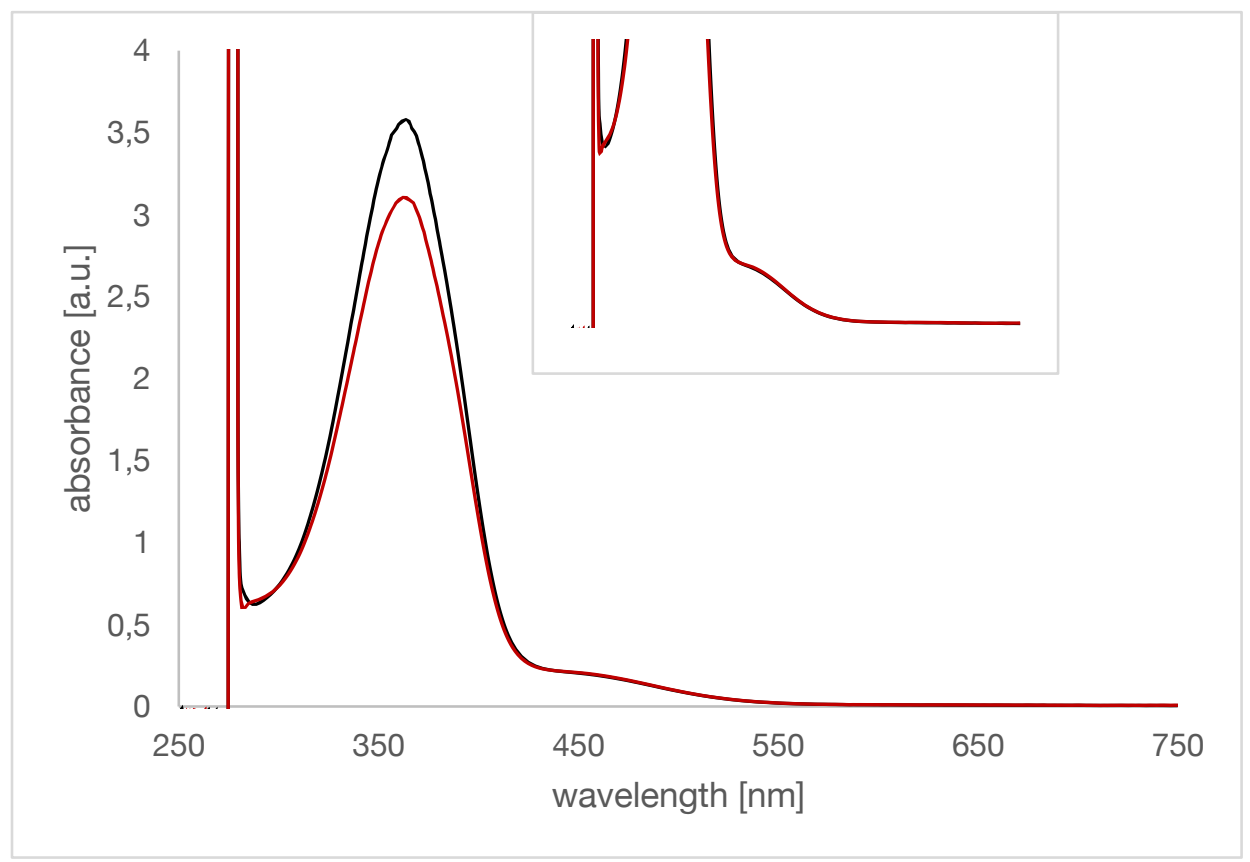

Figure $\mathbf{S 6}$ UV/Vis of 6 in toluene $\left(c=1.3610^{-4} \mathrm{M}\right)$ : daylight, r.t. (black, $\left.E-\mathbf{6}\right)$, daylight, $0{ }^{\circ} \mathrm{C}(\mathrm{red}, E-\mathbf{6})$.

The UV/Vis spectra of catalyst 6 in toluene were first recorded under ambient conditions. After that the samples (in the measuring cuvette) were cooled to $0{ }^{\circ} \mathrm{C}$ and the spectra re-recorded. Using a concentration of $E-6$ as in catalysis $\left(2.0010^{-4} \mathrm{M}\right)$ the detector limit was reached and there is no observable change in absorption of either $\pi \rightarrow \pi^{*}$ transition or $n \rightarrow \pi^{*}$ transition (Fig. S5). A slightly diluted sample of $6\left(1.3610^{-4} \mathrm{M}\right)$ however shows a significant change in the absorption. The signal intensity of $\pi \rightarrow \pi^{*}$ transition is decreased at $0{ }^{\circ} \mathrm{C}$ while the signal intensity of $\mathrm{n} \rightarrow \pi^{*}$ transition does not change at all. This indicates that the solubility of catalyst $E-6$ is significantly lowered at $0^{\circ} \mathrm{C}$, while that of Z-6 is not affected. 


\section{Photochemistry equipment}
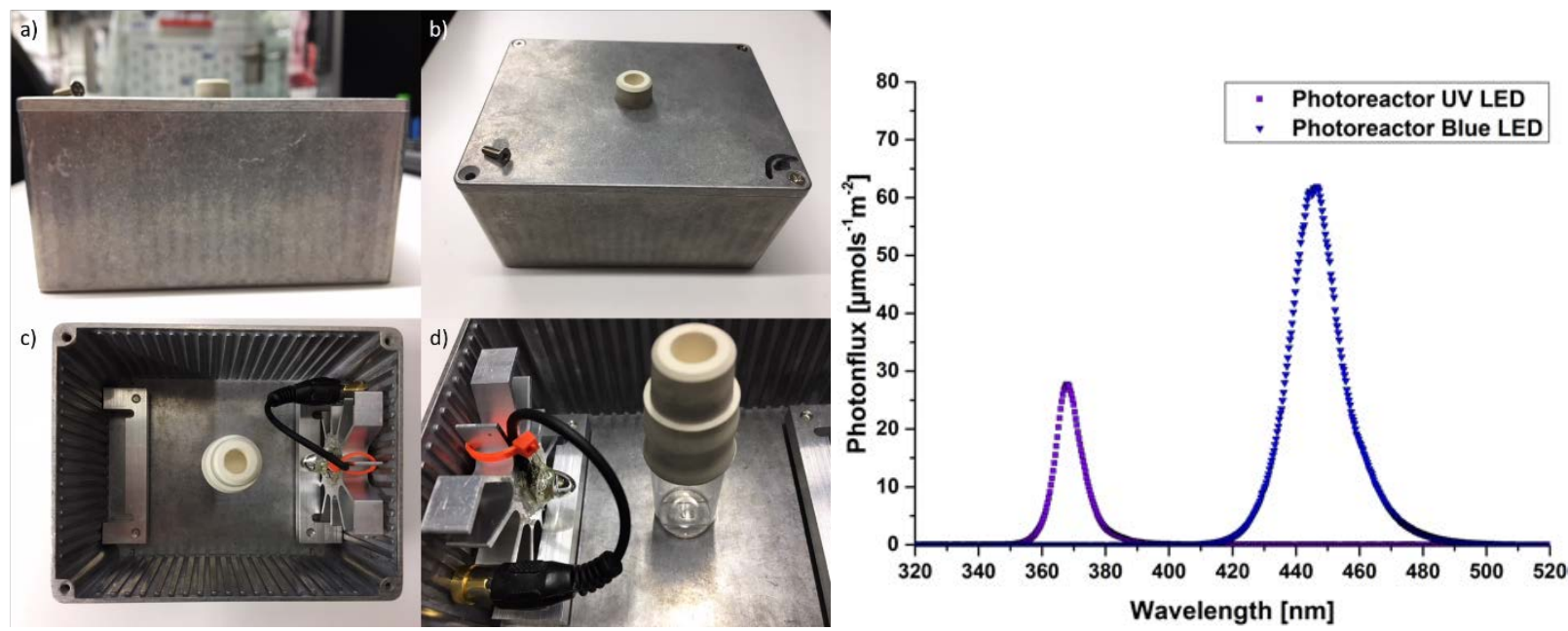

Figure S7. Homemade catalysis device: a) closed lightproof catalysis device b) reaction vessel equipped with septum to inject reactants and reagents c) inlet of the catalysis device with support for the LED and d) close-up view of the LED reaction vessel area: (right) emission spectra of the UV LED (purple) and Blue LED (blue) used within the photoreactor. 


\section{In situ irradiation NMR spectroscopy}

All in situ irradiation NMR experiments were carried out on a Bruker Avance III spectrometer with $600.3 \mathrm{MHz}$ proton frequency, equipped with a $5 \mathrm{~mm}$ Triple Resonance Broadband Probe $(\mathrm{TBI})\left({ }^{1} \mathrm{H},{ }^{13} \mathrm{C}, \mathrm{BB}\right)$ with z-gradients. In situ irradiation NMR experiments were carried out to examine a) timescales of $E \rightarrow Z$ photoisomerization of catalyst 6 upon irradiation with UV light $(\lambda=375 \mathrm{~nm})$ or visible light $(\lambda=448 \mathrm{~nm})$, b) molar fractions of the $E$ - and Z-isomer of 6 in photostationary states during continuous irradiation and $c)$ thermal relaxation rates in different solvents and at different temperatures. Irradiation of the NMR sample within the magnet is applied according to literature. ${ }^{8}$ Samples are prepared in $5 \mathrm{~mm}$ thin walled NMR tubes (Wilmad Labglas). DMF- $d_{7}(99.5$ atom\% D) was purchased from Sigma Aldrich in $0.75 \mathrm{~mL}$ ampullae and used as received. Toluene- $d_{8}(99.5$ atom\% D) was purchased from Armar in $0.75 \mathrm{~mL}$ ampullae and used as received. For irradiation of the NMR sample a UV LED (Nichia NCSU275) was used and coupled to a silica waveguide. ${ }^{8 a}$ Light intensity within the NMR sample was estimated by measuring emission spectra at the tip of the waveguide (BFH 48-1000, Thorlabs) (covered with the coaxial stem insert) with the integration sphere (Avantes AVASPHERE-50-IRRAD) coupled to the UV/Vis spectrometer (Avantes AVASPEC-ULS3648). The signal detected was integrated for $100 \mathrm{~ms}$ and a total of 100 transients were averaged to obtain the final spectrum (Fig. S12). Emission spectra in the reactor and in the NMR tube were integrated over the wavelength range from $340 \mathrm{~nm}$ to $420 \mathrm{~nm}$. Within the photoreactor a total photon flux of $0.314 \mathrm{~mol} \mathrm{~s}^{-1} \mathrm{~m}^{-2}$ is detected while in the NMR tube a total photonflux of $0.044 \mathrm{~mol} \mathrm{~s}^{-1} \mathrm{~m}^{-2}$ is measured.

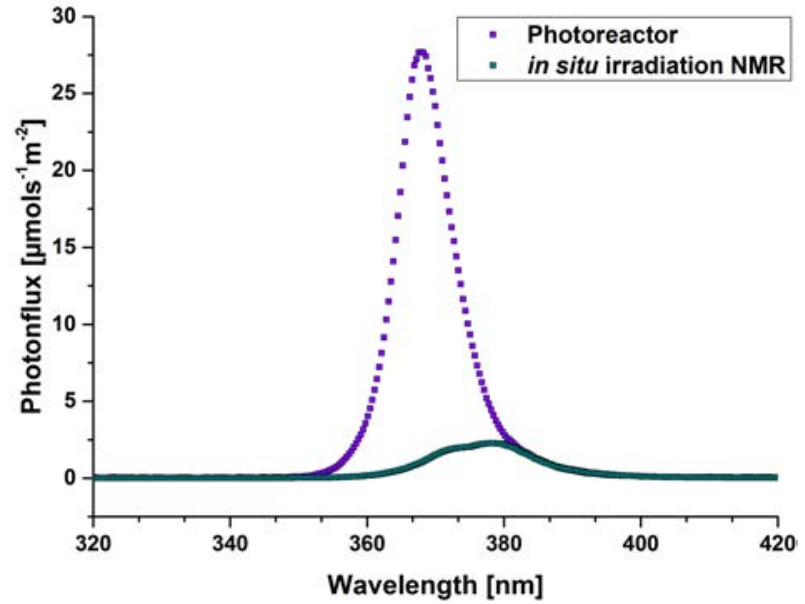

Figure S8: Quantitative emission spectra of the UV LED in the photoreactor used for catalysis (purple) and the tip of the waveguide used for in situ irradiation NMR experiments (cyan).

For in situ irradiation NMR experiments the following solutions of 6 were used:

Table S7: Sample preparation for in situ irradiation NMR experiments.

\begin{tabular}{|c|c|c|c|c|c|c|}
\hline Sample No. & Solvent & Solvent & $\begin{array}{c}\text { Volume solvent } \\
{[\mu \mathrm{L}]}\end{array}$ & $\begin{array}{c}\text { Volume stock solution } \\
{[\mu \mathrm{L}]}\end{array}$ & Mass Catalyst [mg] & $\begin{array}{c}\text { Concentration } \\
{[\mathrm{mM}]}\end{array}$ \\
\hline 1 & Toluene-d $d_{8}$ & Toluene-d $d_{8}$ & 900 & - & $0.6^{1}$ & Theo: 0.8 , ca. $0.2^{2}$ \\
\hline 2 & Toluene- $d_{8}$ & Toluene- $d_{8}$ & 210 & $240^{3}$ & - & 0.2 \\
\hline 3 & Toluene- $d_{8}$ & Toluene- $d_{8}$ & 500 & $125^{4}$ & - & 0.06 \\
\hline 4 & Toluene- $d_{8}$ & Toluene- $d_{8}$ & 500 & $500^{4}$ & - & 0.24 \\
\hline 5 & Toluene- $d_{8}$ & Toluene- $d_{8}$ & 500 & $1000^{4}$ & - & 0.48 \\
\hline 6 & DMF- $d_{7}$ & DMF- $d_{7}$ & 450 & - & 0.5 & 1.38 \\
\hline 7 & $\mathrm{DMF}-d_{7}$ & $\mathrm{DMF}-d_{7}$ & 450 & - & 0.2 & 0.55 \\
\hline
\end{tabular}

${ }^{1}$ Catalyst 6 is not completely dissolved in toluene- $d_{8}$

${ }^{2}$ Concentration estimated by comparison of the residual solvent signals and aromatic signals of catalyst 6

${ }^{3}$ Stock solution of catalyst 6 in toluene- $d_{8}(0.59 \mathrm{mg}, 1950 \mu \mathrm{l}, 375 \mu \mathrm{M})$

${ }^{4}$ Stock solution of catalyst 6 in DCM $(0.97 \mathrm{mg}, 5 \mathrm{ml}, 240 \mu \mathrm{M})$ with subsequent removal of the solvent.

For the preparation of the samples with low concentrations two approaches were used. Approach one was the preparation of a stock solution by dissolving $0.59 \mathrm{mg}$ of catalyst 6 in $1.95 \mathrm{~mL}$ in toluene- $d_{8}$. After the corresponding volume was transferred into a $5 \mathrm{~mm}$ NMR tube, it was diluted with additional toluene- $d_{8}$ to obtain a sample with the required concentration. To reduce weighting errors and avoiding the usage of large amounts of deuterated solvent a second approach was used. In this case of the stock solution in DCM was prepared first by dissolving $0.97 \mathrm{mg}$ of catalyst 6 in $5.00 \mathrm{~mL}$ dichloromethane. After transfer of this stock solution into the NMR tube the solvent was removed under reduced pressure before toluene- $d_{8}$ was added.

Afterwards a coaxial stem insert (Wilmad labglass), which contains the roughened tip of the silica waveguide (Thorlabs), is inserted into the solution in the NMR tube until the tip of the insert reaches the bottom of the NMR tube. 
The other end of the waveguide is coupled a) to a Nichia SMD LED UV NCSU275 for irradiation of the sample with UV light $(\lambda=375 \mathrm{~nm})$, b) to a Nichia SMD LED NVSU233B $(\lambda=365 \mathrm{~nm})$ or $\mathrm{c})$ to a Luxeon $Z$ royal blue LED $(\lambda=448 \mathrm{~nm})$.

Photochemical $E \rightarrow Z$ and $Z \rightarrow E$ isomerization as well as thermal $Z \rightarrow E$ isomerization is monitored with series of ${ }^{1} \mathrm{H}$ NMR spectra. These spectra series are acquired as pseudo-2D experiments. The temporal resolution of these spectra was chosen to be between 10 and 60 s. Within pseudo-2D experiments ${ }^{1} \mathrm{H}$ NMR spectra were acquired with $64 \mathrm{k}$ data points over a spectral width of $20.54 \mathrm{ppm}$ within an acquisition time of $2.66 \mathrm{~s}$. The resulting data planes were apodized by exponential multiplication $(\mathrm{lb}=0.3 \mathrm{~Hz})$ and Fourier transformed in the direct dimension. After phase and baseline correction the resulting data planes are converted into a series of $1 \mathrm{D}{ }^{1} \mathrm{H}$ NMR spectra by using a macro. Automatic integration of the relevant regions is carried out with the Bruker Topspin macro intser.

All pulse sequences used were taken from the Bruker library.

HSQC experiments (hsqcetgpsp.2) were acquired with 1200 points in the direct dimension over a spectral width of 15 ppm within an acquisition time of $67 \mathrm{~ms}$. In the indirect dimension 512 data points were acquired over a spectral width of $165 \mathrm{ppm}$. For each increment 16 scans were accumulated. Between each transient a relaxation delay of $1.5 \mathrm{~s}$ was used. The delay for evolution of ${ }^{1} \mathrm{~J} \mathrm{CH}$ couplings was optimized for a coupling constant of ${ }^{1} \mathrm{~J}_{\mathrm{CH}}=145 \mathrm{~Hz}$. Prior to Fourier transformation the data plane was zerofilled to $2 \mathrm{k}$ point in the direct and $1 \mathrm{k}$ points in the indirect dimension. Apodization was carried out with squared sine functions $(\mathrm{SSB}=2)$ in both dimensions. Fourier transformed spectra were phase and baseline corrected manually.

HMBC experiments (hmbcgpndqf) were acquired with 4k data points in the direct dimension over a spectral width of 18 ppm within an acquisition time of $189 \mathrm{~ms}$. In the indirect dimension 512 data points were acquired over a spectral width of $222 \mathrm{ppm}$. For each increment 32 transients were accumulated with a relaxation delay of $1.5 \mathrm{~s}$ between each transient. The delay for evolution of the $\mathrm{C}-\mathrm{H}$ long range couplings was optimized for a coupling constant of $8 \mathrm{~Hz}$. Prior to Fourier transformation the data plan was zerofilled to $8 \mathrm{k}$ points in the direct and $1 \mathrm{k}$ points in the indirect dimension and apodized with sine function $(\mathrm{SSB}=0)$ in both dimensions. 


\section{Examination of photostationary states during irradiation with UV or blue light}

NMR spectra of catalyst 6 in toluene- $d_{6}$ show a severe dependency of the proton chemical shifts and line widths on temperature indicating aggregation. This is observed already without irradiation and prohibits a detailed investigation of $\mathbf{6}$ at low temperature.

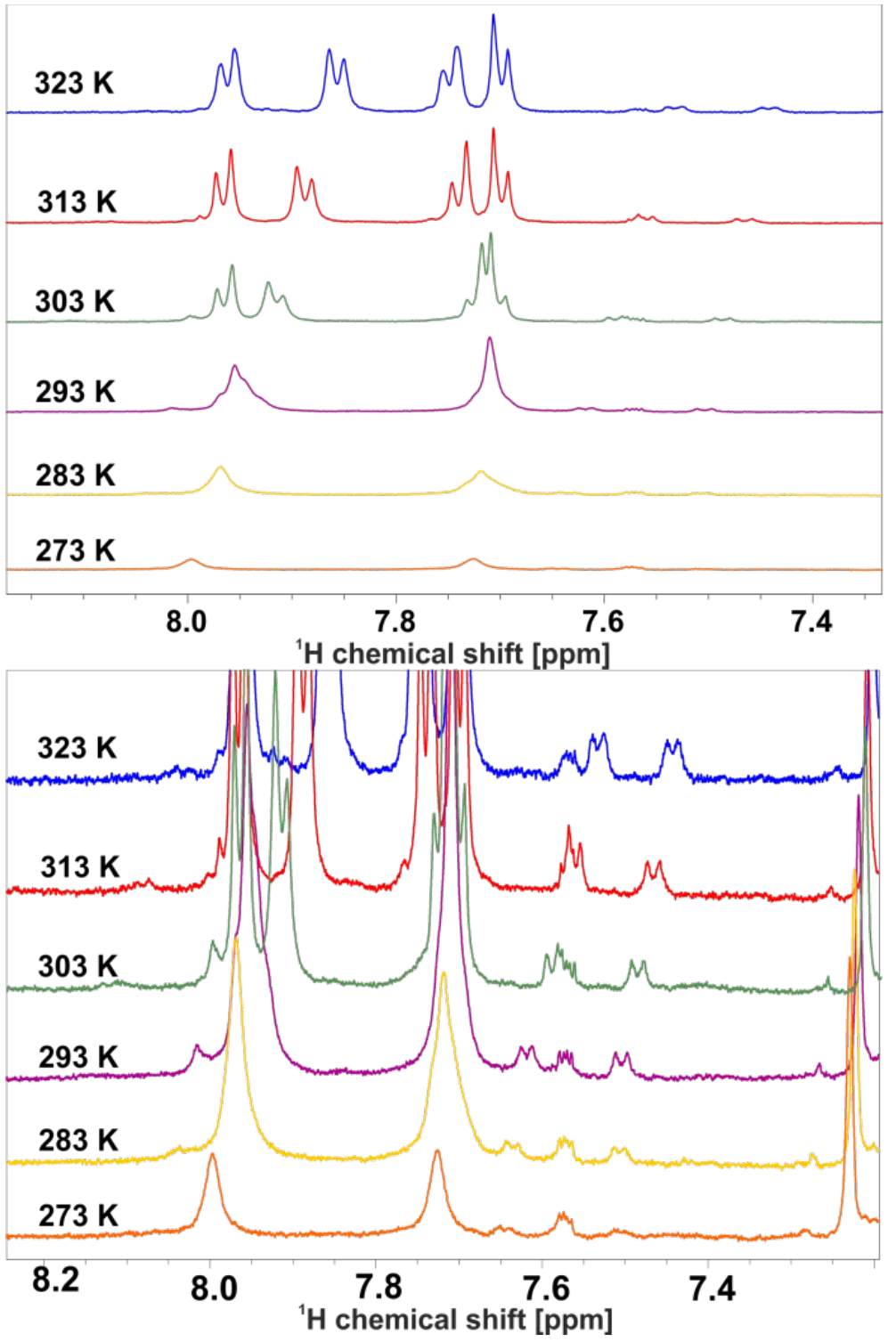

Figure S9: (top) Aromatic signals of the E-isomer of catalyst 6 (sample 1). ${ }^{1} \mathrm{H}$ NMR spectra (600 $\mathrm{MHz}$ proton resonance frequency) in toluene- $d_{8}$ at different temperatures with temperature dependent chemical shifts and decaying signal intensity indicating aggregation. ${ }^{9} \mathrm{All}$ spectra were referenced on the residual $-\mathrm{CHD}_{2}$ signal of toluene. (bottom) Magnification of the NMR spectra with the aromatic resonances of the Z-isomer of catalyst 6, which show similar temperature dependent proton chemical shifts. 


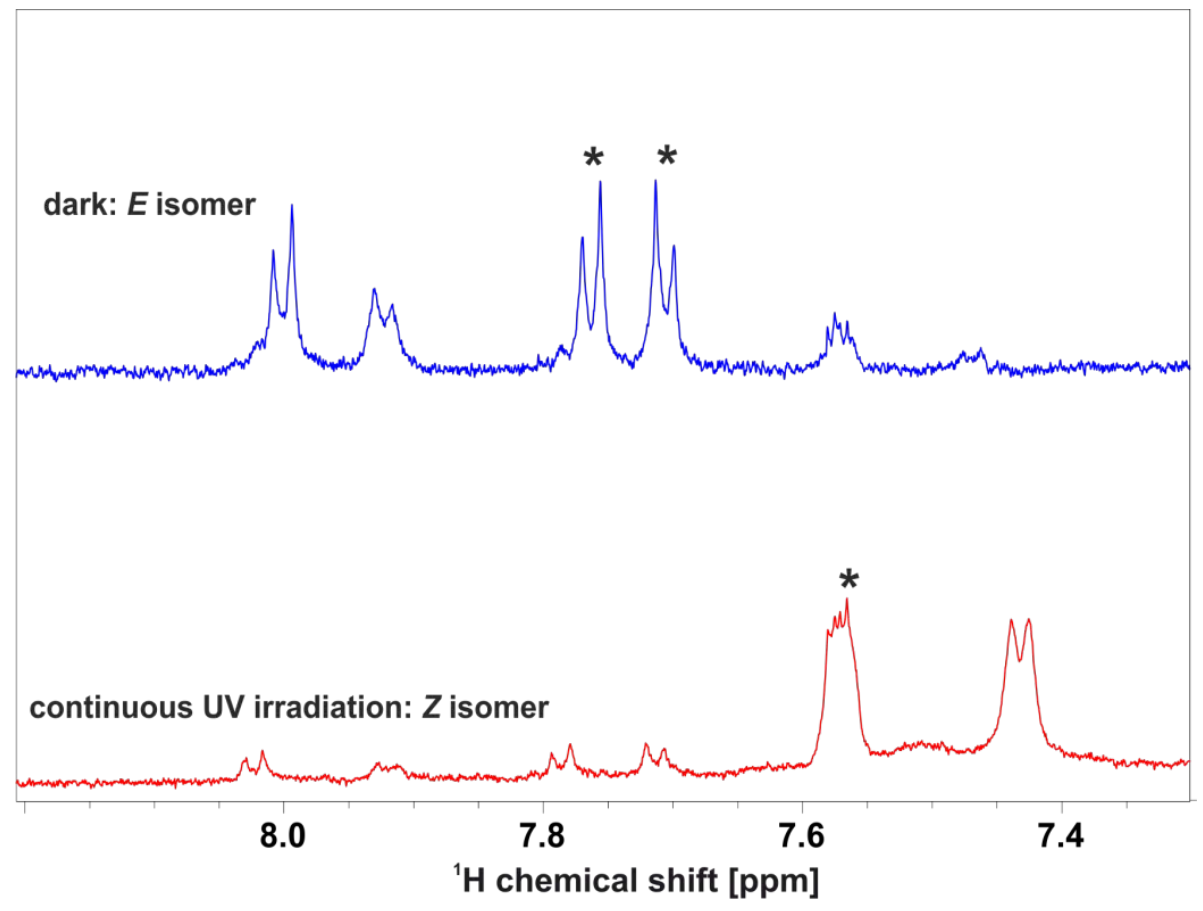

Figure S10: Aromatic region ${ }^{1} \mathrm{H}$ NMR spectra (600 MHz proton resonance frequency) of catalyst 6 in toluene- $d_{8}$ (sample 1) prior to (upper trace) and during continuous (lower trace) irradiation with UV light $(\lambda=375 \mathrm{~nm})$ at $300 \mathrm{~K}$. Integration of the aromatic resonances (asterisked signals) shown is used to calculate the molar fractions of both isomers in the dark state as well as in the photo stationary state.

Due to incomplete dissolution of catalyst 6 in toluene- $d_{8}$ with a theoretical concentration of $0.8 \mathrm{mM}$ a sample with $0.2 \mathrm{mM}$ (which is equal to our catalyst loading in the catalysis) was prepared (sample 2). ${ }^{1} \mathrm{H}$ NMR spectra of this sample also show a severe dependency of the proton chemical shift on the temperature. Photo-isomerization of the catalyst at $273 \mathrm{~K}$ with UV light ( $365 \mathrm{~nm})$ is unaffected.

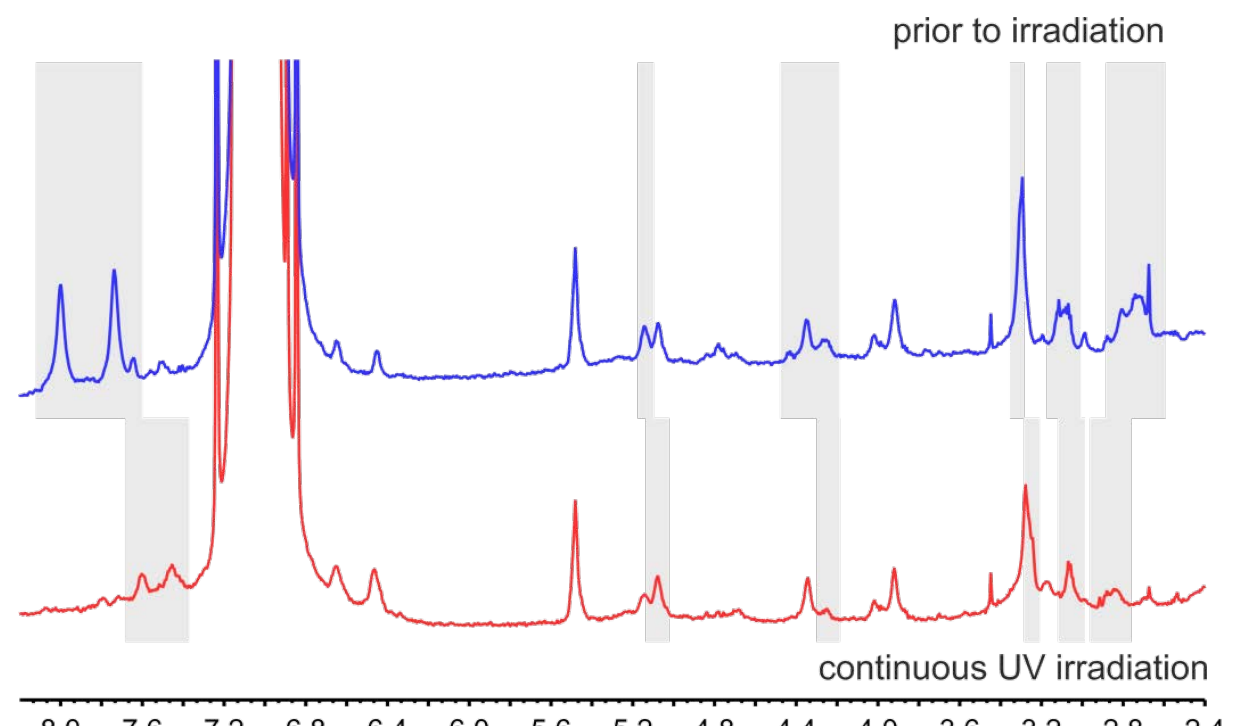

${ }^{1} \mathrm{H}$ chemical shift $[\mathrm{ppm}]$

Figure S11: ${ }^{1} \mathrm{H}$ NMR spectra $\left(600 \mathrm{MHz}\right.$ proton resonance frequency) of catalyst 6 in toluene- $d_{8}$ (sample 2) prior to (upper trace) and during continuous (lower trace) irradiation with UV light $(\lambda=365 \mathrm{~nm})$ at $273 \mathrm{~K}$. Areas highlighted in grey show signals of the catalyst indicating a successful isomerization from $E$ into its $Z$ isomer.

Additionally, NMR spectra of lower concentrated samples were investigated to get a deeper insight into the chemical shift dependency on the concentration. A stock solution of catalyst 6 in toluene- $d_{8}$ was prepared and samples with increasing concentrations were measured at different temperatures (samples 3-5). These show essentially the same dependencies. 

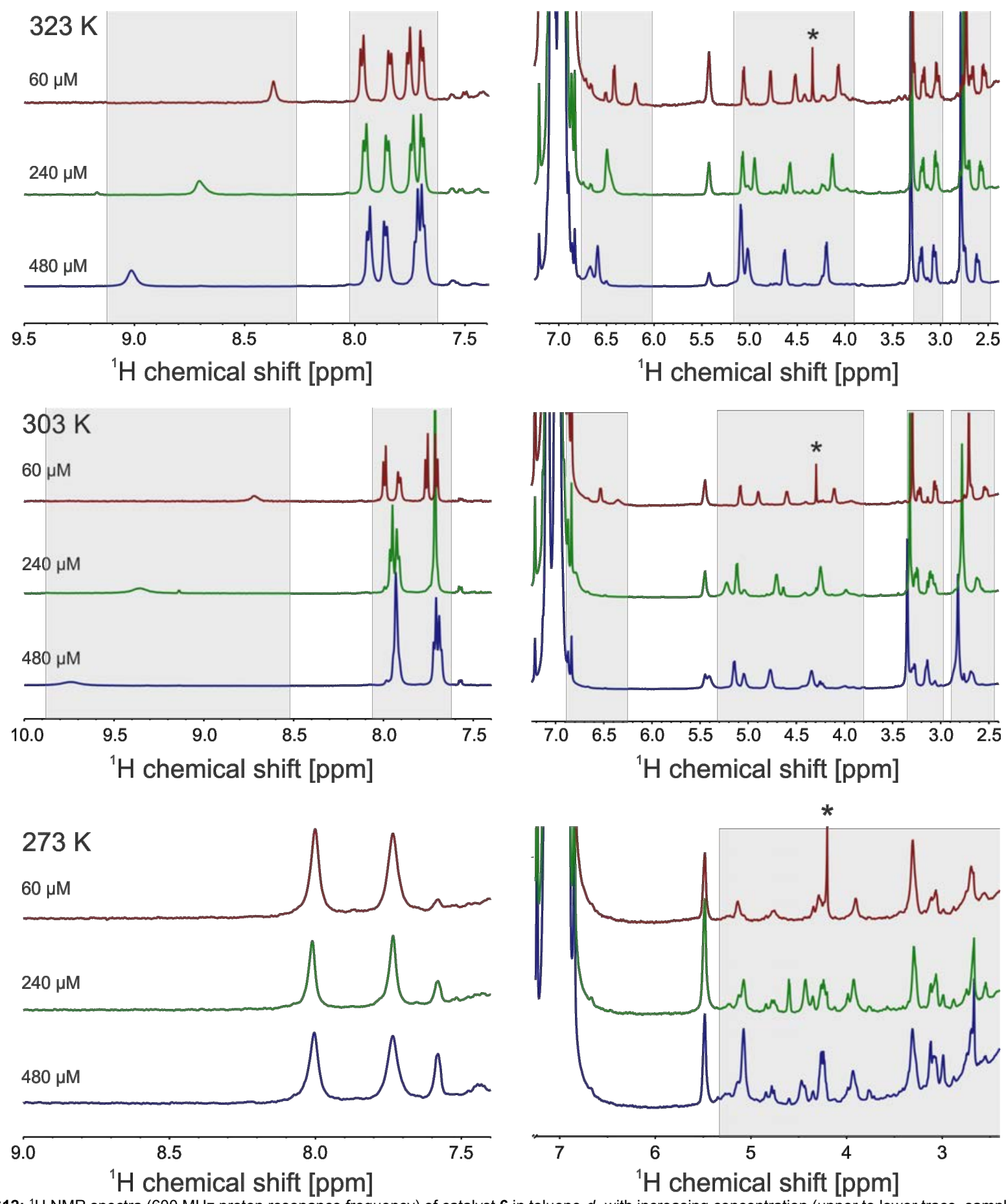

Figure S12: ${ }^{1} \mathrm{H}$ NMR spectra ( $600 \mathrm{MHz}$ proton resonance frequency) of catalyst 6 in toluene- $d_{8}$ with increasing concentration (upper to lower trace, samples $3-5$ ) at different temperatures $(323,303$ and $273 \mathrm{~K})$. Areas highlighted in grey show signals of the catalyst shifting due to concentration dependency. Asterisked signals are residues of DCM only occurring at $60 \mu \mathrm{M}$.

Although the chemical shift dependency on concentration can clearly be seen at higher temperatures so that even individual signals can be tracked, it is much harder to discern at $273 \mathrm{~K}$. Furthermore, it can be seen that even at a concentration of $60 \mu \mathrm{M}$, where no signs of precipitation are visible to the naked eye line broadening is observed in NMR spectra. To investigate whether this effect is potentially dependent on the photoisomer of catalyst 6 further experiments with continuous irradiation with UV-light and different temperatures were performed. 


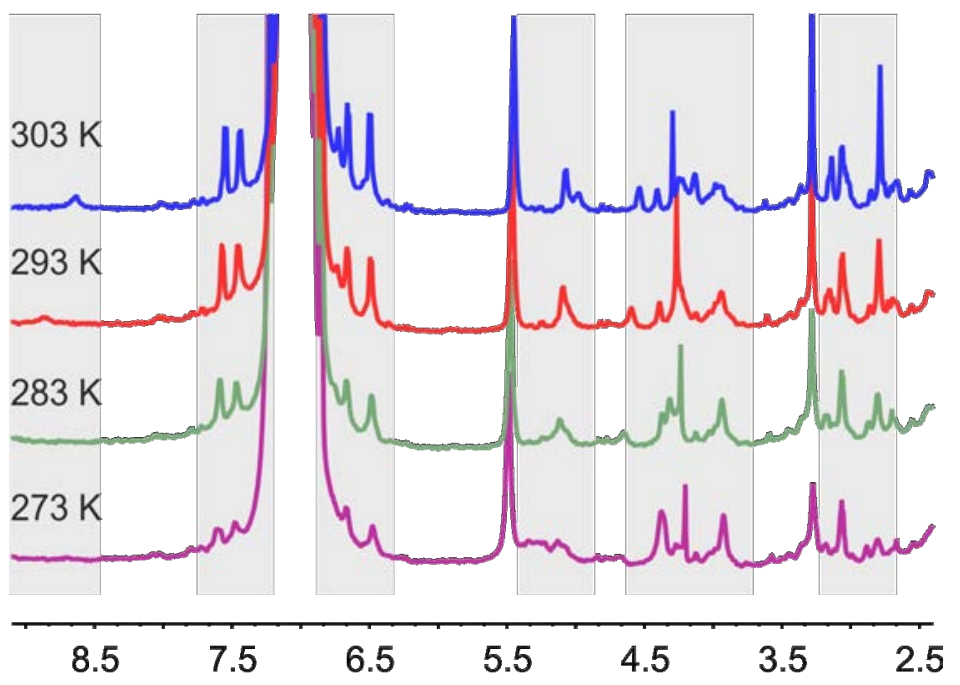

${ }^{1} \mathrm{H}$ chemical shift [ppm]

Figure S13: ${ }^{1} \mathrm{H}$ NMR spectra $\left(600 \mathrm{MHz}\right.$ proton resonance frequency) of catalyst 6 in toluene- $d_{8}(60 \mu \mathrm{M}$, sample 3$)$ at different temperatures under continuous irradiation with UV light (365 nm). Areas highlighted in grey show signals of the catalyst shifting and line broadening indicating aggregation.

Both $E$ - and Z-Isomer show a chemical shift dependency on the temperature. However, the photo-isomerization of the catalyst is still given with similar photo stationary states ( $90 \%$ of Z-6 present) and no signs of precipitation are observable.

After continuous irradiation with UV light $(365 \mathrm{~nm})$ the thermal relaxation was investigated. Therefore, ${ }^{1} \mathrm{H}$-NMR spectra under continuous irradiation and after a thermal relaxation time of 2 hours were performed. These spectra show negligible difference in signal intensities indicating that thermal relaxation occurs on the timescale of hours or even longer.

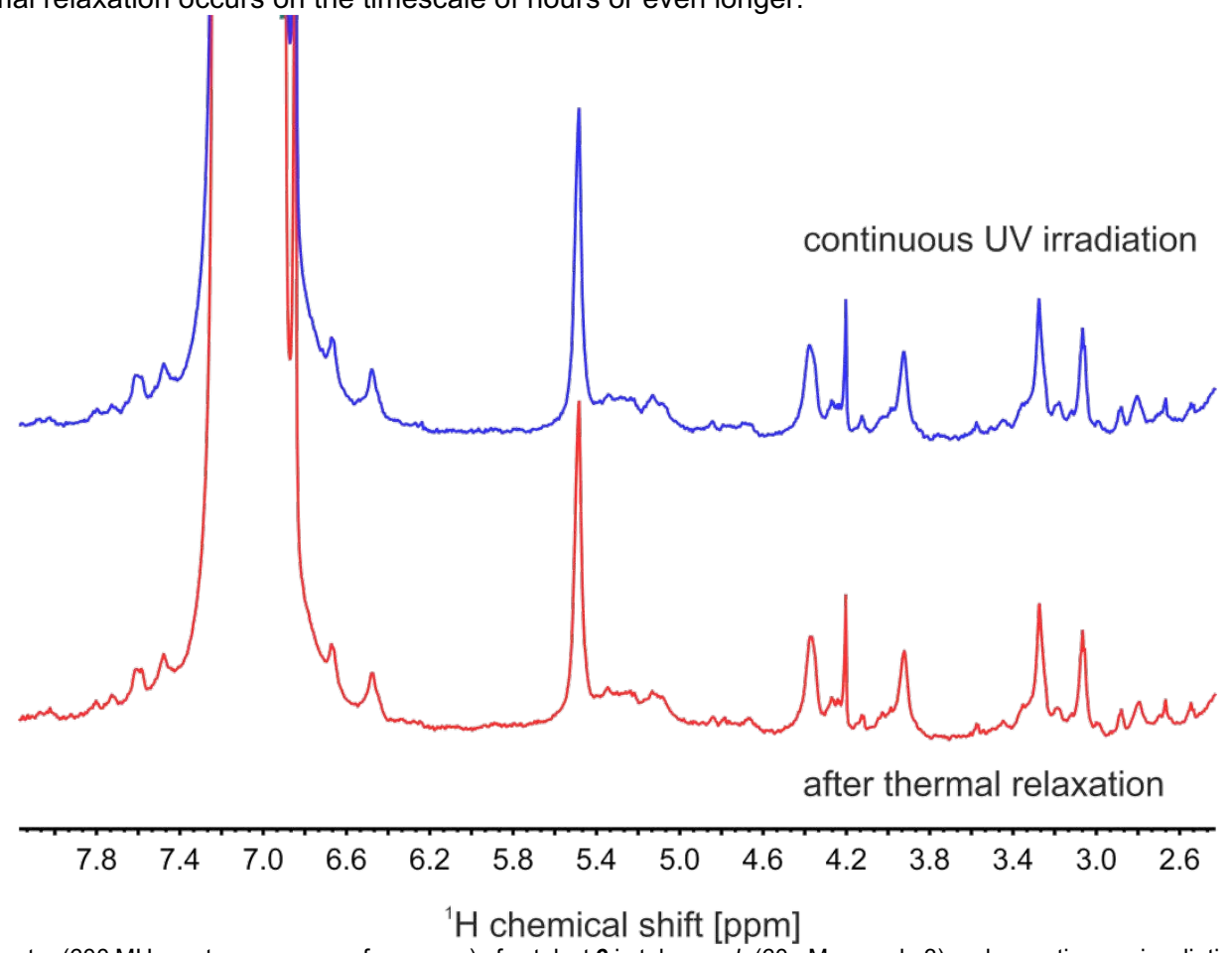

Figure S14: ${ }^{1} \mathrm{H}$ NMR spectra ( $600 \mathrm{MHz}$ proton resonance frequency) of catalyst 6 in toluene- $d_{8}(60 \mu \mathrm{M}$, sample 3$)$ under continuous irradiation with UV light (365 nm) and after a thermal relaxation time of 2 hours. A negligible difference in the signal intensities indicates no measurable thermal relaxation within this period of time.

To overcome issues concerning low solubility and concentration together with temperature dependences for further investigations DMF was used as solvent. In contrast to toluene- $d_{8}$ the solubility of 6 in DMF- $d_{7}$ is sufficiently large to prepare samples on the mM concentration scale. This allows for measurement with a smaller number of consecutive transients and with a higher signal-to-noise ratio. In DMFd the proton chemical shifts of 6 are independent from concentration. By using in situ irradiation NMR spectroscopy ${ }^{1} \mathrm{H}$ NMR spectra of the $E$ - and Z-isomers of catalyst 6 can be obtained (Fig. S15), either by measuring in the dark (E-isomer) or during continuous irradiation with UV light (Z-isomer). 


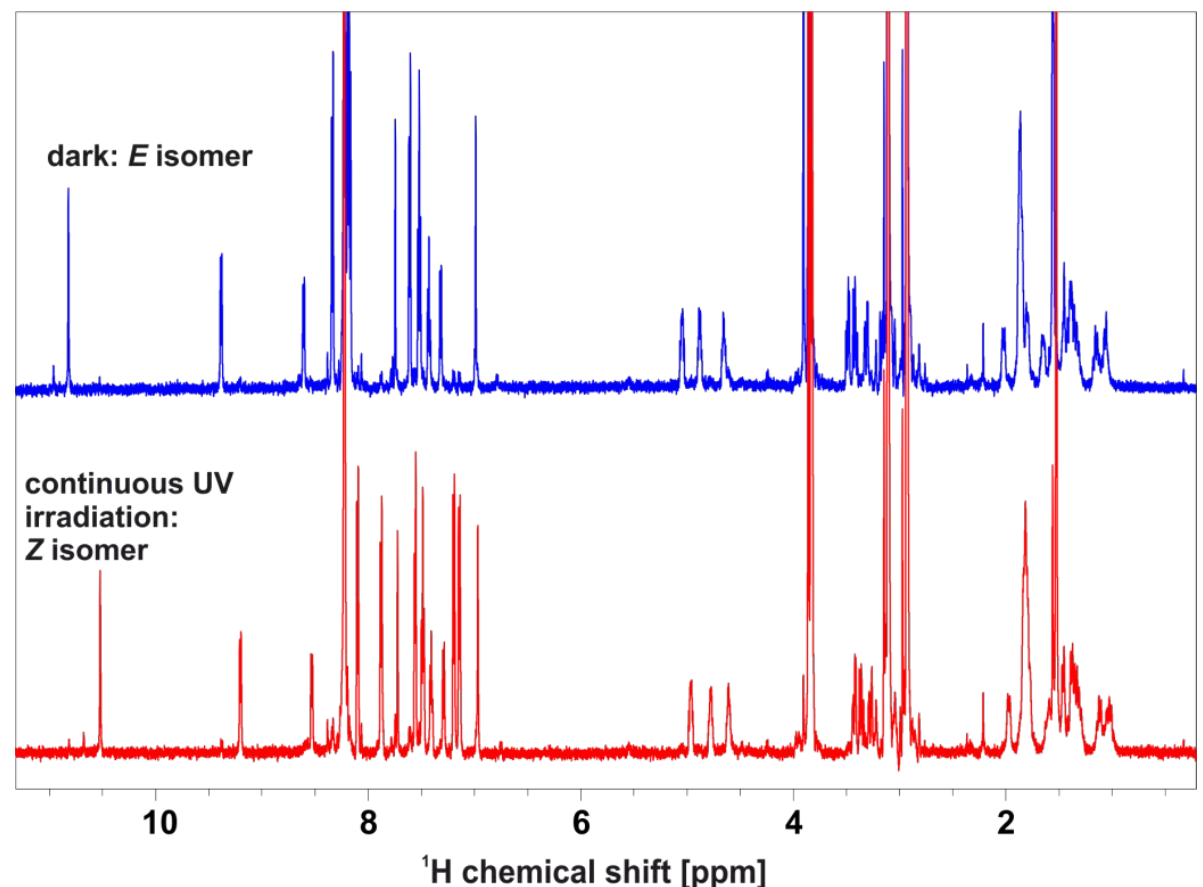

Figure S15: ${ }^{1} \mathrm{H}$ NMR spectra $\left(600 \mathrm{MHz}\right.$ proton resonance frequency) of a $1.3 \mathrm{mM}$ solution of catalyst 6 in DMF- $d_{7}$ (sample 6 ) at $273 \mathrm{~K}$ prior to irradiation (upper trace) and during continuous irradiation (lower trace) with UV light $(\lambda=375 \mathrm{~nm})$.

Mixtures of both isomers can be prepared by using short irradiation intervals instead of continuous irradiation with UV light. For the Eisomer an unusual signal pattern for the azobenzene moiety is observed. While for the carbonyl substituted aromatic ring the typical resonance pattern of two doublets for $p$-disubstituted aromatic rings is observed, the proton resonances of the amino substituted ring occur as broad pseudo-singlet. This isochronicity, which could be understood in terms of similar substituents, is not observed for the proton resonances within the Z-isomer though. As expected, the proton chemical shifts of the azobenzene moiety in the Z-isomer are significantly shielded compared to the $E$-isomer. Furthermore the proton resonances of the phenyl side chain, the protons in the $\alpha$ position of the four peptide bonds as well as of the histidine residue are shielded in the more compact Z-isomer compared to the $E$ isomer, indicating that the chemical and electronic environment of the backbone and these side chains alters during photoisomerization.
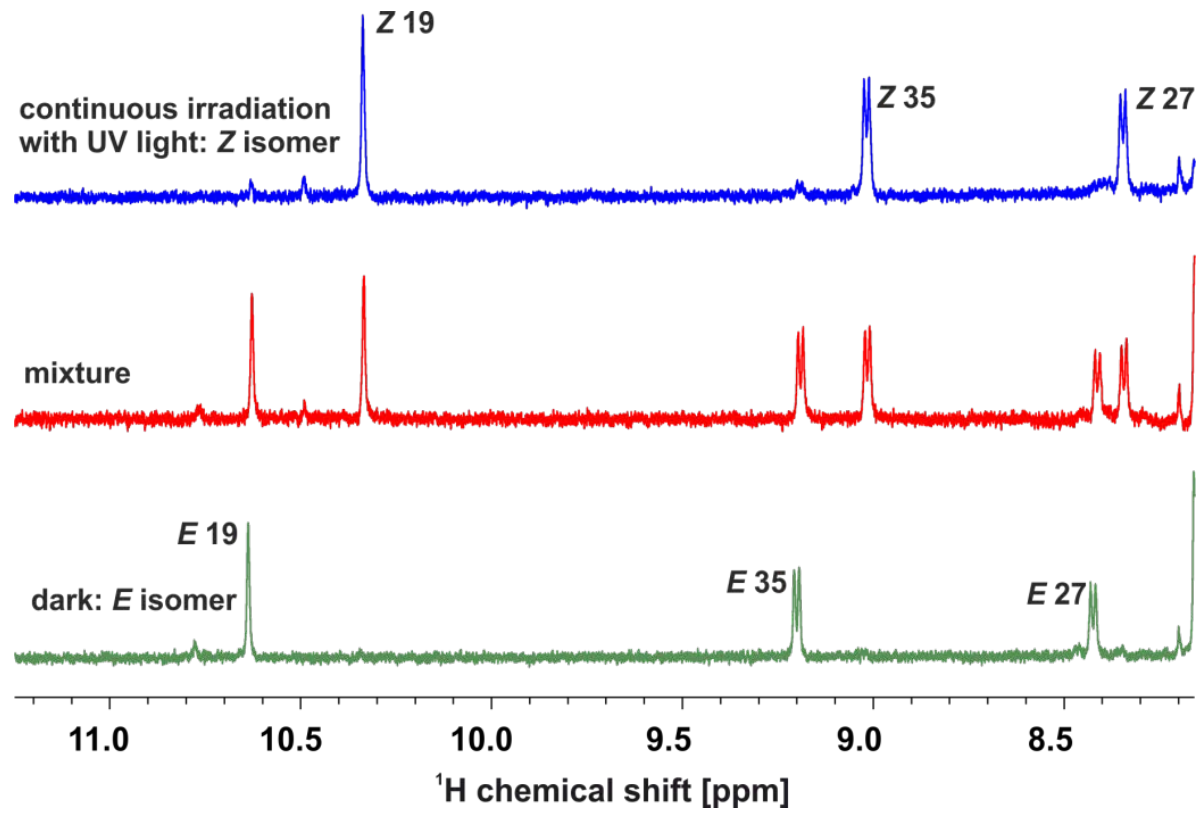

Figure S16: Amide signals in ${ }^{1} \mathrm{H}$ NMR spectra ( $600 \mathrm{MHz}$ proton resonance frequency) of a $1.3 \mathrm{mM}$ solution of catalyst 6 in DMF- $d_{7}$ (sample 6$)$ at $273 \mathrm{~K}$ prior to irradiation (lower trace), of a mixture of $E$ - and $Z$-isomer produced by an short irradiation interval and during continuous irradiation (upper trace) with UV light ( $\lambda=$ $375 \mathrm{~nm}$ ). 


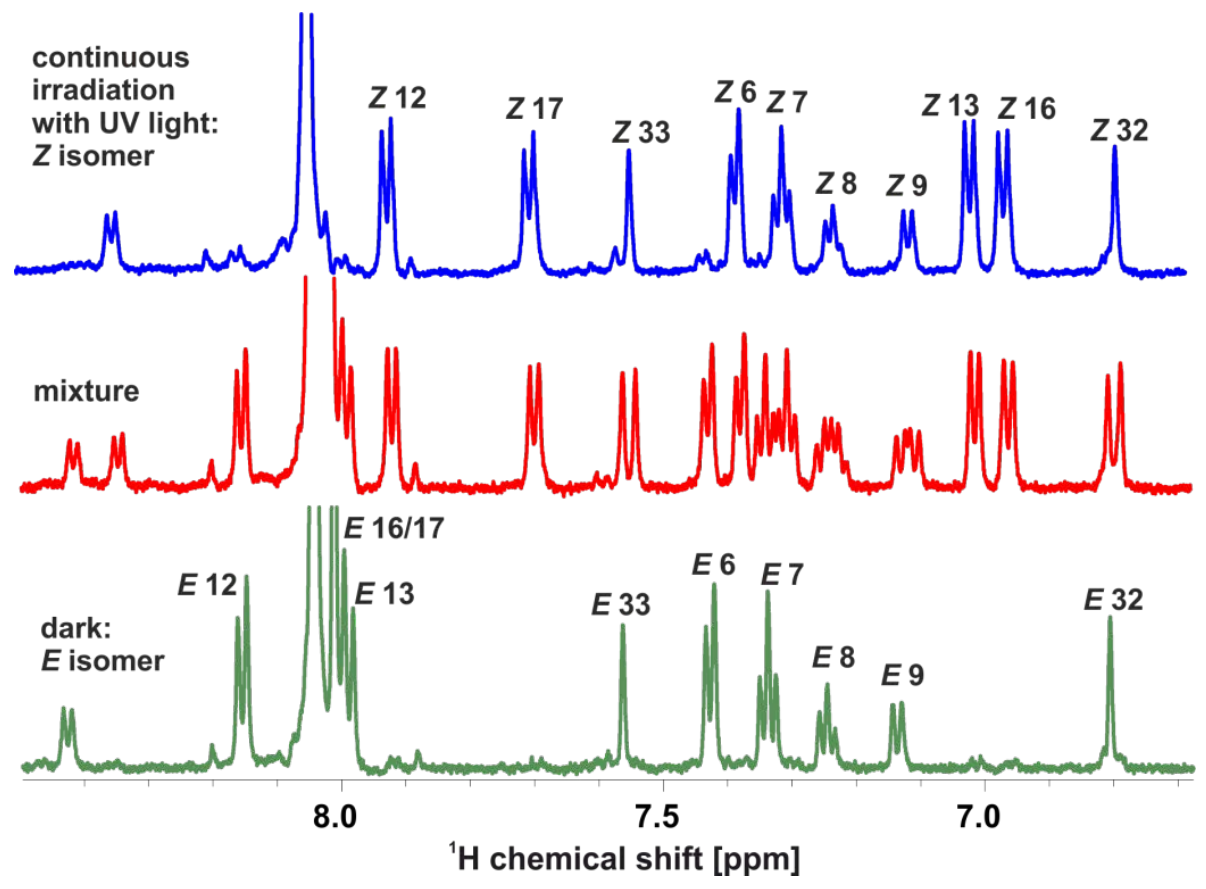

Figure S17: Aromatic resonances in ${ }^{1} \mathrm{H}$ NMR spectra of a $1.3 \mathrm{mM}$ solution of catalyst 6 in DMF- $d_{7}$ (sample 6 ) at $273 \mathrm{~K}$ prior to irradiation (lower trace), of a mixture of $E$ - and Z-isomer produced by an short irradiation interval and during continuous irradiation (upper trace) with $U V$ light $(\lambda=375 \mathrm{~nm}$ ).

continuous irradiation with UV light: $Z$ isomer
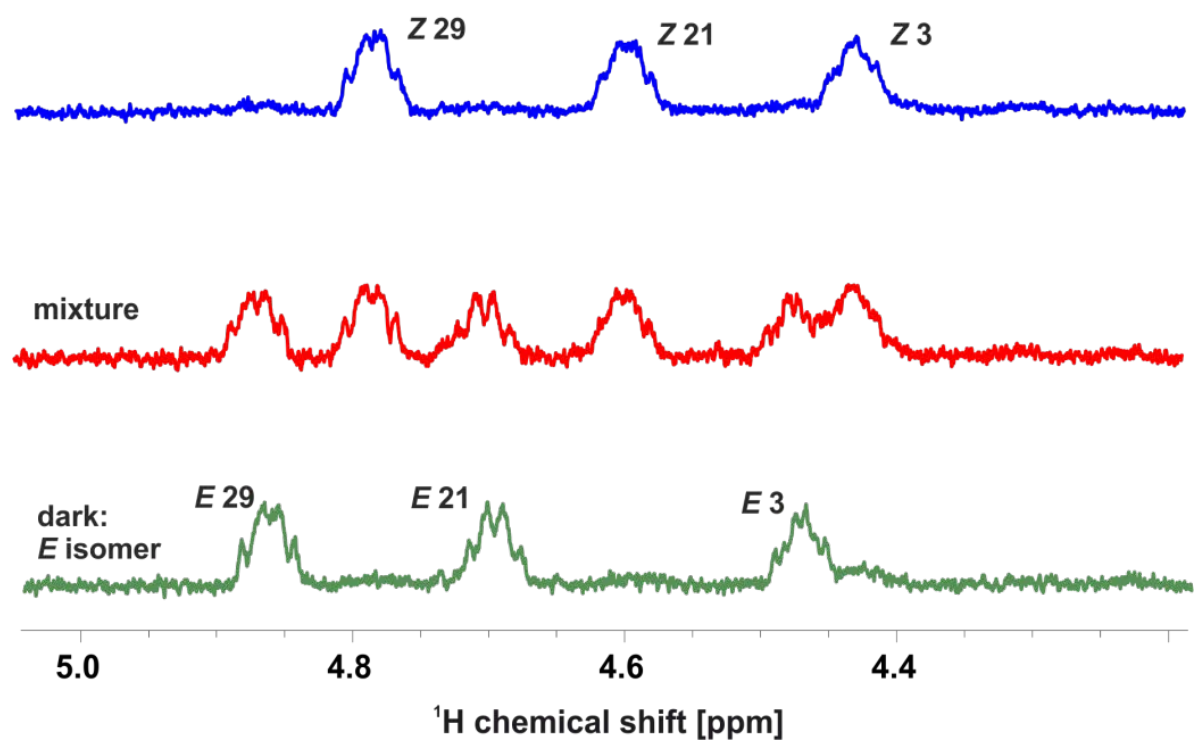

Figure S18: Resonances of protons in the $\alpha$ position of the peptide bonds in ${ }^{1} \mathrm{H}$ NMR spectra (600 MHz proton resonance frequency) of a $1.3 \mathrm{mM}$ solution of catalyst 6 in DMF- $d_{7}$ at (sample 6) $273 \mathrm{~K}$ prior to irradiation (lower trace), of a mixture of $E$ - and Z-isomer produced by an short irradiation interval and during continuous irradiation (upper trace) with UV light $(\lambda=375 \mathrm{~nm})$. 
For a full signal assignment COSY, HSQC and HMBC spectra of a mixture of both isomers were acquired.

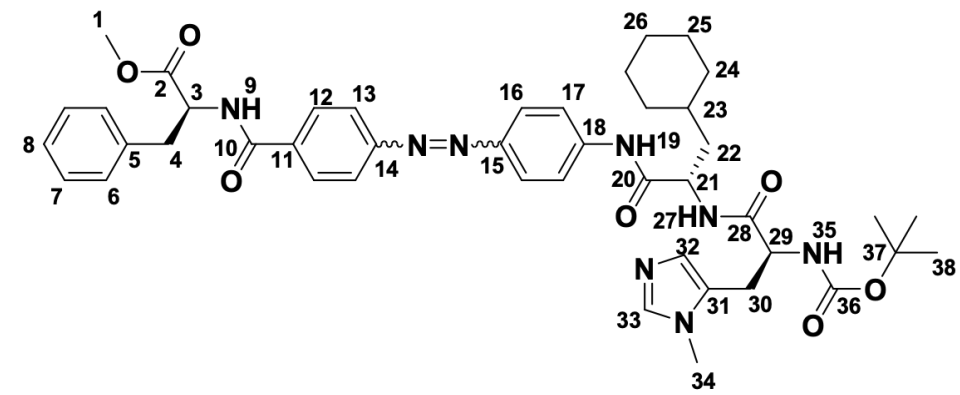

Figure S19: Numbering of catalyst 6 for assignment of ${ }^{1} \mathrm{H}$ and ${ }^{13} \mathrm{C}$ resonances.

Table S8: ${ }^{1} \mathrm{H}$ and ${ }^{13} \mathrm{C}$ chemical shift assignment for the $E$ - and Z-isomer of catalyst 6 in DMF- $d_{7}$ at $273 \mathrm{~K}$.

\begin{tabular}{|c|c|c|c|c|}
\hline \multirow[b]{2}{*}{ Atom number } & \multicolumn{2}{|c|}{$E$-isomer } & \multicolumn{2}{|c|}{ Z-isomer } \\
\hline & $\delta_{H}[p p m]$ & $\delta_{c}[p p m]$ & $\delta_{H}[p p m]$ & $\delta_{c}[p p m]$ \\
\hline 1 & 3.73 & 51.9 & 3.68 & 51.6 \\
\hline 2 & - & 172 & - & 172 \\
\hline 3 & 4.48 & 53.9 & 4.43 & 53.9 \\
\hline 4 & $3.1-2.9$ & 26.4 & $3.1-2.9$ & 26.4 \\
\hline 5 & - & 138.2 & - & 138.2 \\
\hline 6 & 7.43 & 129.3 & 7.38 & 129.2 \\
\hline 7 & 7.34 & 128.4 & 7.30 & 128.4 \\
\hline 8 & 7.25 & 126.7 & 7.22 & 126.7 \\
\hline 9 & 7.14 & - & 7.10 & - \\
\hline 10 & - & 166.2 & - & 166.1 \\
\hline 11 & - & 135.8 & - & -1 \\
\hline 12 & 8.16 & 128.9 & 7.92 & 128.5 \\
\hline 13 & 7.99 & 122.5 & 7.01 & 119.7 \\
\hline 14 & - & 154.1 & $\cdot$ & -1 \\
\hline 15 & - & 148.4 & - & -1 \\
\hline 16 & $8.02^{2}$ & 124 & 6.96 & 122.2 \\
\hline 17 & $8.02^{2}$ & 119.7 & 7.67 & 119.1 \\
\hline 18 & - & 143.3 & - & -1 \\
\hline 19 & 10.63 & - & 10.34 & - \\
\hline 20 & - & 172.4 & - & 172 \\
\hline 21 & 4.7 & 51.9 & 4.59 & 51.8 \\
\hline 22 & 1.73 & 39.7 & 1.62 & 39.7 \\
\hline $23-26$ & $1.8-0.8$ & $39.8-26.1$ & - & - \\
\hline 27 & 8.42 & - & 8.40 & - \\
\hline 28 & - & -1 & - & -1 \\
\hline 29 & 4.87 & 54.9 & 4.78 & 54.9 \\
\hline 30 & 3.3 & 36.5 & 3.2 & 36.5 \\
\hline 31 & - & -1 & - & -1 \\
\hline
\end{tabular}




$\begin{array}{lcccc}32 & 6.81 & 127.6 & 6.79 & 127.6 \\ 33 & 7.56 & 137.9 & 7.54 & 137.9 \\ 34 & 3.65 & 137.9 & 3.65 & 137.9 \\ 35 & 9.2 & - & 9.02 & - \\ 36 & - & 166 & - & 166 \\ 37 & - & 79.8 & - & 79.8 \\ 38 & 1.38 & 27.9 & 1.34 & 27.9\end{array}$

${ }^{1}$ No correlation detected

${ }^{2}$ Protons 16 and 17 show isochronic shifts and strong coupling within the E-isomer, hence a pseudo-singlet is detected.

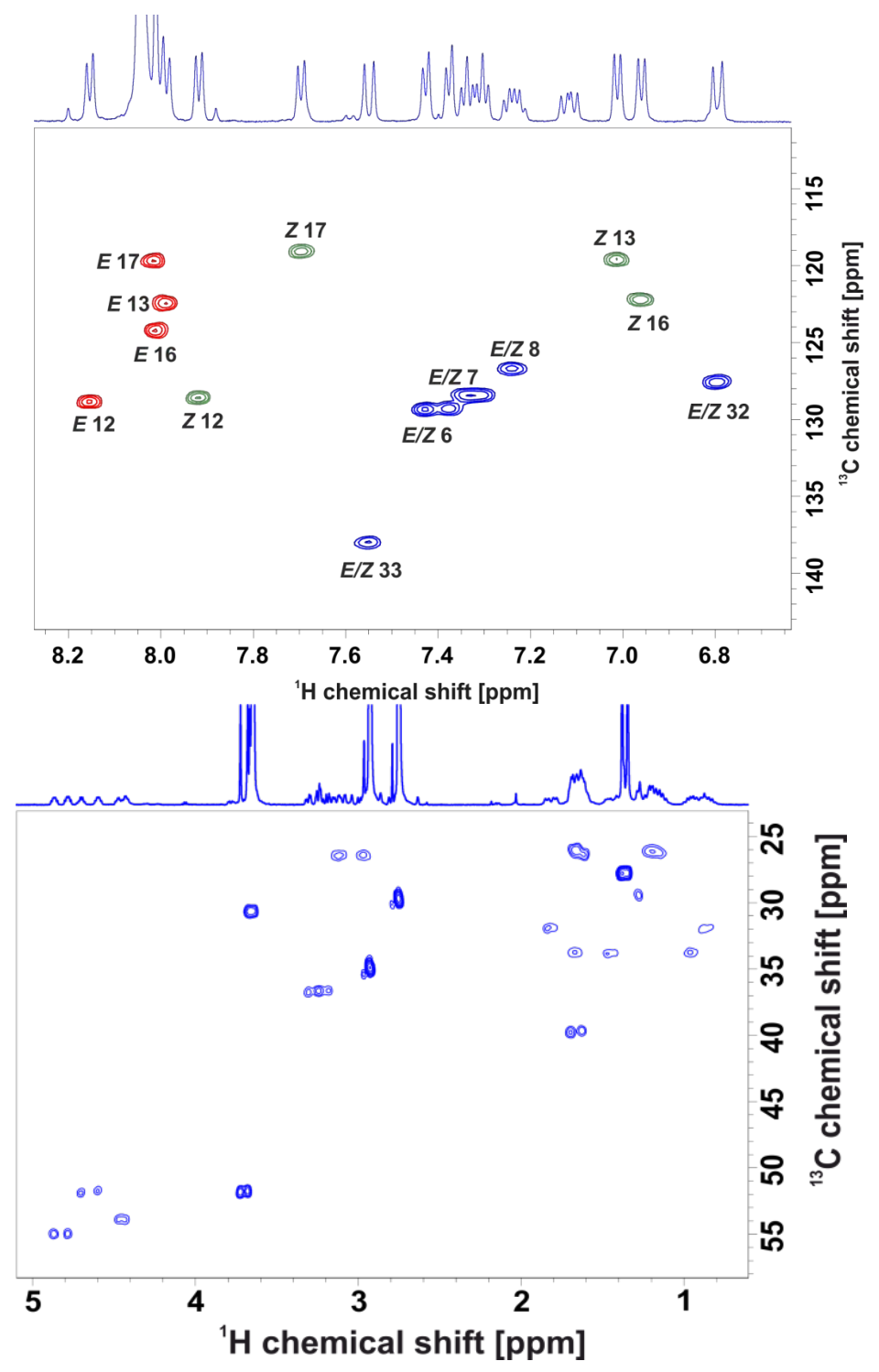

Figure S20: (top) part of an HSQC spectrum ( $600 \mathrm{MHz}$ proton resonance frequency) of a mixture with $E$ - and Z-isomer of catalyst 6 in DMF- $d_{6}$ at $273 \mathrm{~K}$ showing C$\mathrm{H}$ correlations of the azobenzene moieties within the $E$ isomer (red) and within the $Z$ isomer (green) as well as the $\mathrm{CH}$ correlations within the phenyl residue and histidine. (bottom) part of an HSQC spectrum of a mixture with $E$ and $Z$ isomer of catalyst 6 in DMF- $d_{6}$ at $273 \mathrm{~K}$ showing $\mathrm{CH}$ correlations of the aliphatic protons. 


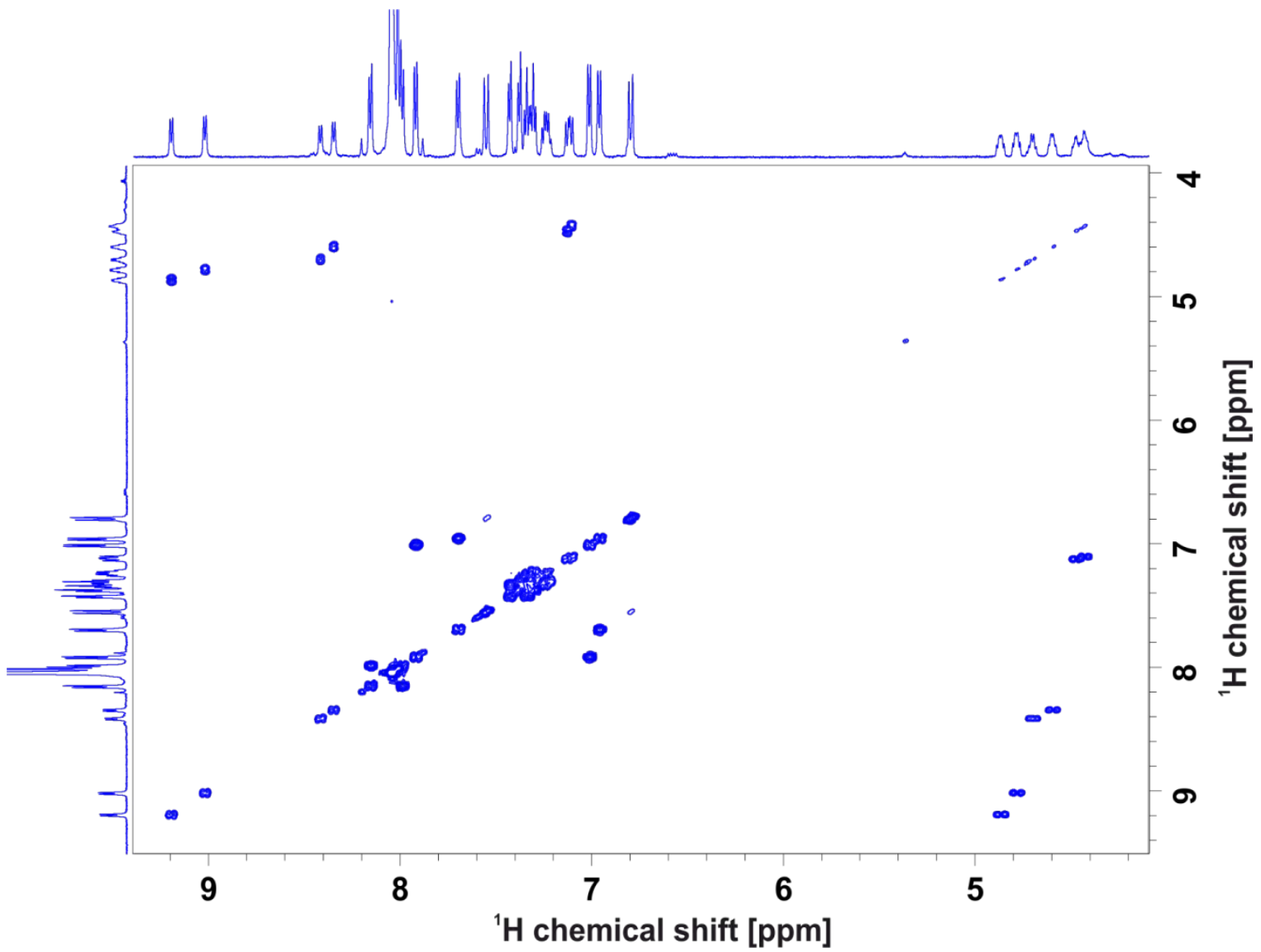

Figure S21: COSY spectrum ( $600 \mathrm{MHz}$ proton resonance frequency) of the mixture with $\mathrm{E}$ - and Z-isomer of catalyst 6 in DMF- $d_{6}$ at $273 \mathrm{~K}$ showing $\mathrm{H}$-H correlations of the azobenzene moieties within the isomers as well as $\mathrm{H}-\mathrm{H}$ correlations of the amide protons with the protons in the $\alpha$ position of the amide bonds within the backbone. 


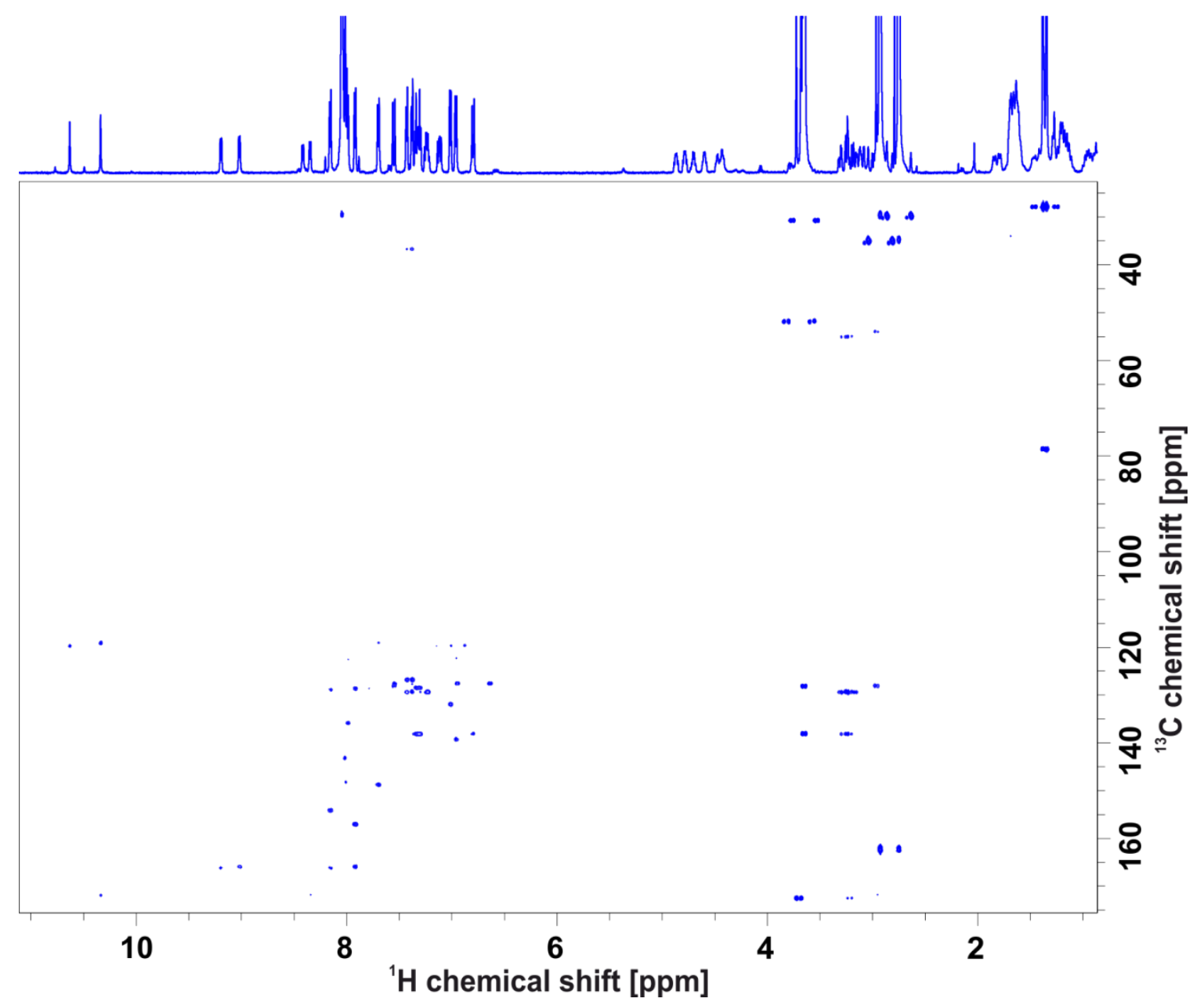

Figure S22: HMBC spectrum (600 MHz proton resonance frequency) of a mixture with $E$ - and Z-isomer of catalyst 6 in DMF- $d_{7}$ at $273 \mathrm{~K}$ showing $\mathrm{C}-\mathrm{H}$ long range correlations in both isomers.

\section{Concentration time profiles during irradiation of catalyst 6 with UV light, blue light and thermal fading}

For catalyst 6 in DMF- $d_{7}$ concentration time profiles were extracted from ${ }^{1} \mathrm{H}$ NMR spectra series during continuous irradiation with UV light $(\lambda=375 \mathrm{~nm})$ until a photo stationary state is reached. Afterwards the UV LED is turned off and the sample was either irradiated with blue light $(\lambda=448 \mathrm{~nm})$ until a stationary state is observed or the concentration decay of Z-isomer is monitored during thermal relaxation. The molar fraction of both isomers is calculated from the integrals of two - NH signals of the corresponding isomers by:

$$
\begin{aligned}
& x_{Z}=\frac{I_{\text {amide }-H, Z}}{\left(I_{\text {amide }-H, Z}+I_{\text {amide }-H, E)}\right.} \\
& x_{E}=\frac{I_{\text {amide }-H, E}}{\left(I_{\text {amide }-H, Z}+I_{\text {amide }-H, E}\right)}
\end{aligned}
$$



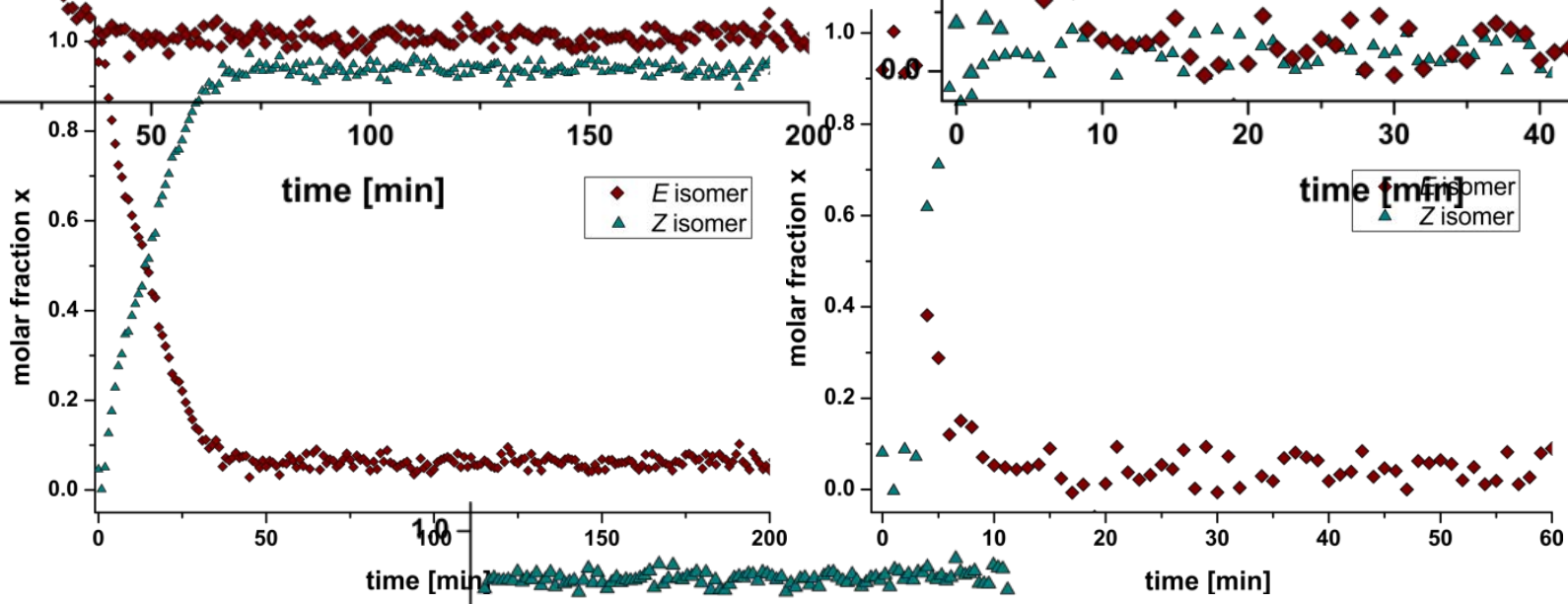

Figure S23: Molar fraction vs. time profiles for $E$ - (cyan) and Z- (red) isomer of a $1.3 \mathrm{mM}$ solution (left) and a $0.7 \mathrm{mM}$ solution (right) of catalyst 6 in DMF- $d_{7}$ during continuous irradiation with UV light $(\lambda=375 \mathrm{~nm})$ at $273 \mathrm{~K}$. Time dependent concentrations are calculated using integrals of amide protons of the $E$ - and Z-isomer extracted from ${ }^{1} \mathrm{H}$ NMR spectra series with a temporal resolution of 1 spectrum per minute.

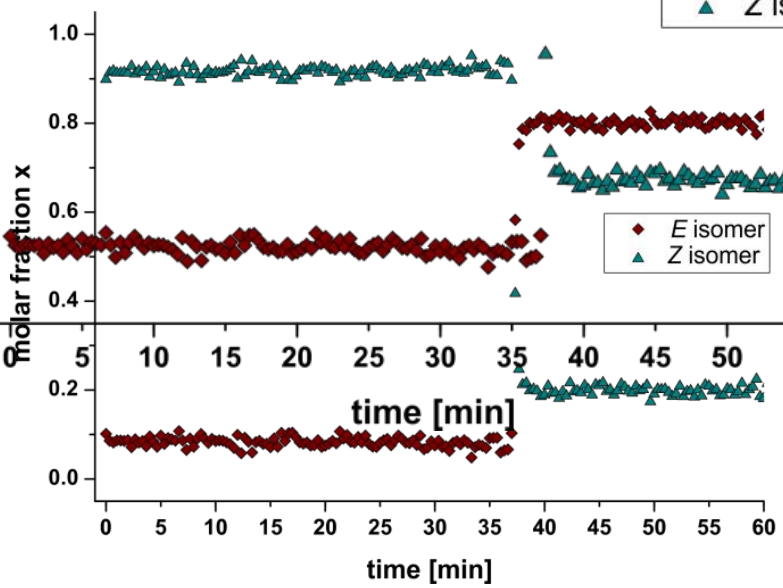

Figure S24: Molar fraction vs. time profiles for $E$ - (cyan) and Z- (red) isomer of a $1.3 \mathrm{mM}$ solution of catalyst 6 in DMF- $d_{6}$ during thermal fading after irradiation with UV light $(\lambda=375 \mathrm{~nm})$ followed by continuous irradiation with blue light $(\lambda=448 \mathrm{~nm})$ at $273 \mathrm{~K}$. Time dependent concentrations are calculated using integrals of amide protons of the $E$ - and $Z$ - isomer extracted from ${ }^{1} \mathrm{H}$ NMR spectra series with a temporal resolution of 1 spectrum per minute.

UV/Vis experiments and in situ irradiation NMR experiments indicate that continuous irradiation with UV or blue light induces photochemical $E \rightarrow Z$ as well as $Z \rightarrow E$ isomerization. In case of irradiation with UV light photochemical $E \rightarrow Z$ exceeds $Z \rightarrow E$ isomerization, so that a photo stationary state with the Z-isomer as major component is found. Irradiation with blue light $(\lambda=448 \mathrm{~nm})$ lead to a photostationary state with $20 \%$ of the $Z$-isomer, so that it has to be assumed, that at this wavelength photochemical $E \rightarrow Z$ conversion is either due to excitation of the $n \rightarrow \pi^{*}$ transition or is induced by hitting the flank of the $\pi \rightarrow \pi^{*}$ of the $E$-isomer.<smiles>[R]c1ccc(N=Nc2ccc([R2])cc2)cc1</smiles>

Scheme S1: Conversion of $E$ - and Z-isomers of catalyst 6 , including photochemical $E \rightarrow Z$ and $Z \rightarrow E$ isomerization with the corresponding photo quantum yields $\varphi$ superimposed by the thermal $Z \rightarrow E$ isomerization with the rate constant $k$.

The rate constant $k_{\mathrm{ZE}}$ of the thermal fading reaction can be determined by observing the concentration decay of the $Z$-isomer after turning off the UV LED. It is assumed that a thermal $E \rightarrow Z$ isomerization plays a minor role, so that following rate laws can be applied.

$$
\frac{d[Z]}{d t}=-k_{Z E} \cdot[Z]
$$


After rearrangement and integration in the borders of $t=0$ and $t$, a monoexponential equation is obtained.

$$
\begin{gathered}
\frac{d[Z]}{[Z]}=-k_{Z E} \cdot d t \\
\int_{0}^{t} \frac{d[Z]}{[Z]}=-k_{Z E} \cdot \int_{0}^{t} d t \\
\ln [Z]_{t}-\ln [Z]_{0}=-k_{Z E} \cdot(t-0) \\
{[Z]_{t}=[Z]_{0} \cdot e^{-k_{Z E} \cdot t}}
\end{gathered}
$$

For the fitting procedure time dependent concentrations of $Z$ are plotted and the rate constant $k$ ZE is varied until a minimum for the sum $\chi^{2}$ of the time dependent residuals is found.

$$
\chi^{2}=\sum_{0}^{t}\left([Z]_{N M R}-[Z]_{\text {calc }}\right)^{2}
$$
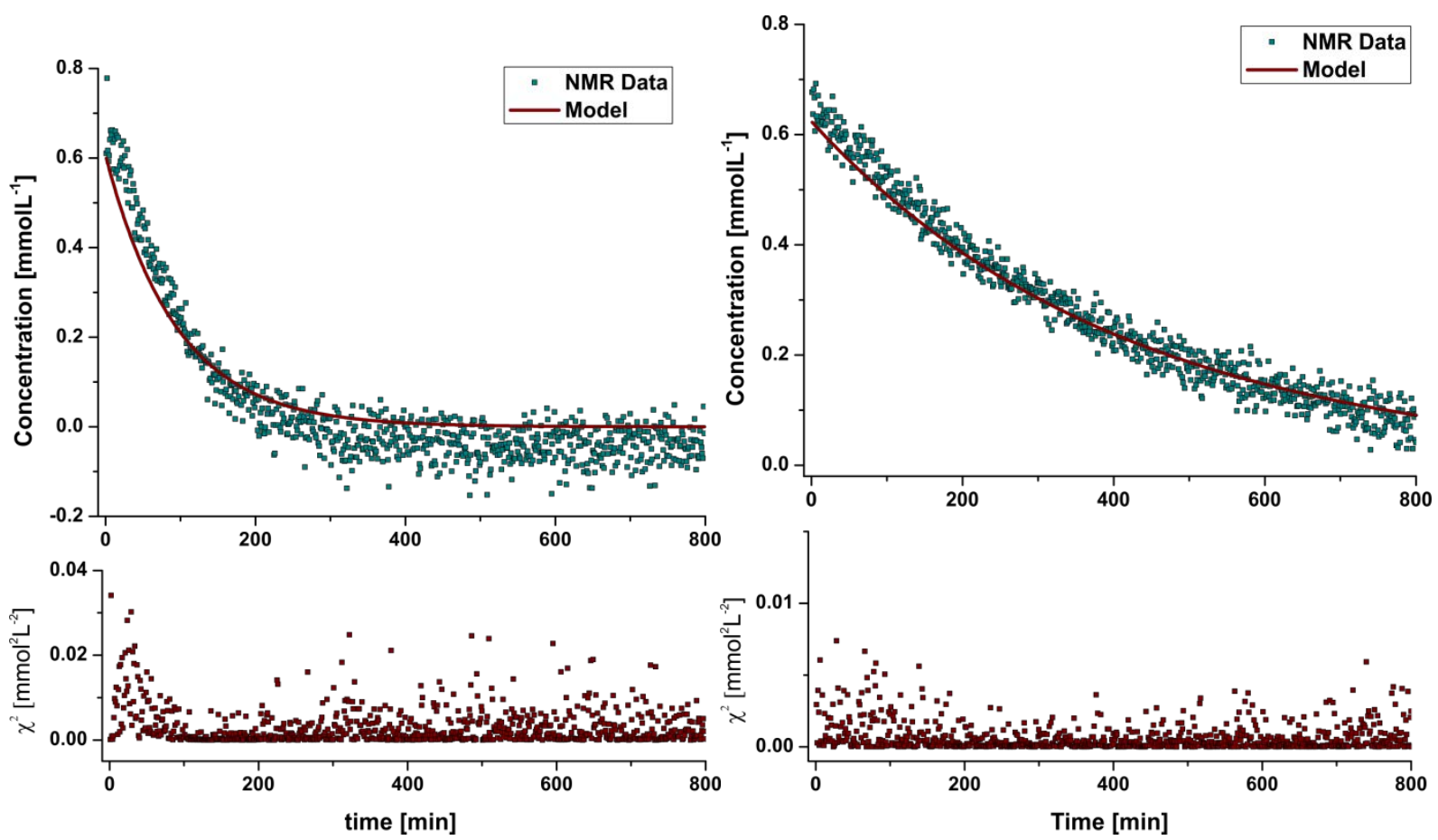

Figure S25: Concentration profile of the Z-isomer in a $0.7 \mathrm{mM}$ solution of catalyst 6 in DMF- $d_{7}$ after turning off the UV LED $(\lambda=375 \mathrm{~nm})$ at $323 \mathrm{~K}$ (left) and $310 \mathrm{~K}$ (right). Monoexponential fits are shown as red lines. The squared residuals are plotted below the concentration profile. 

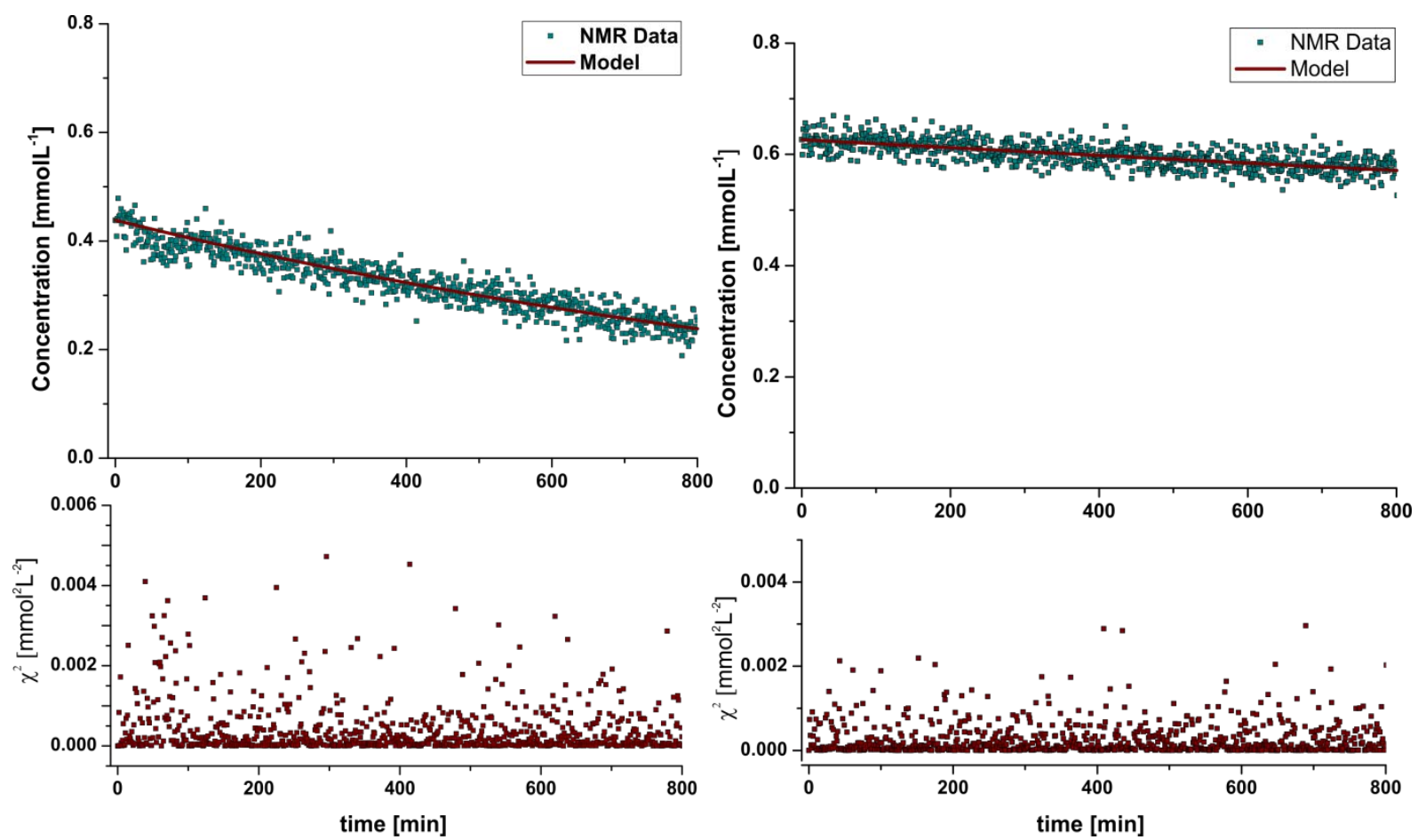

Figure S26: Concentration profile of the Z-isomer in a $0.7 \mathrm{mM}$ solution of catalyst 6 in DMF- $d_{7}$ after turning off the UV LED ( $\lambda=375 \mathrm{~nm}$ ) at $300 \mathrm{~K}$ (left) and $286 \mathrm{~K}$ (right). Monoexponential fits are shown as red lines. The squared residuals are plotted below the concentration profile.
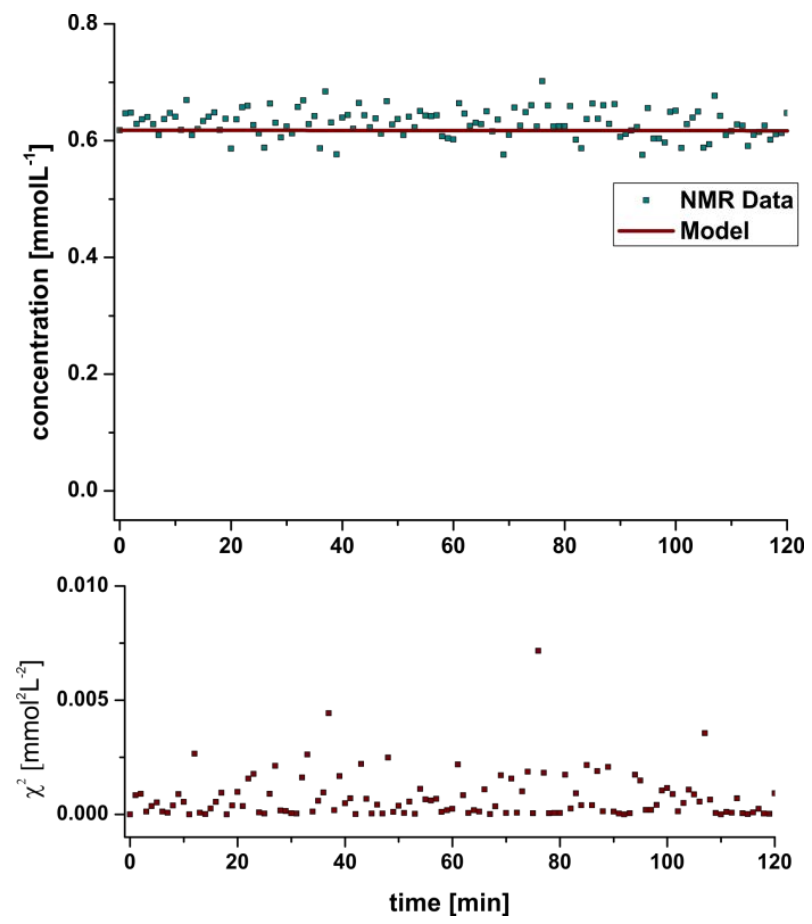

Figure S27: Concentration profile of the Z-isomer in a $0.7 \mathrm{mM}$ solution of catalyst 6 in DMF- $d_{7}$ after turning off the UV LED $(\lambda=375 \mathrm{~nm})$ at $273 \mathrm{~K}$. Monoexponential fits are shown as red lines. The squared residuals are plotted below the concentration profile.

As thermal $Z \rightarrow E$ isomerization at $300 \mathrm{~K}, 286 \mathrm{~K}$ and $273 \mathrm{~K}$ occurs on a slow timescale, the decay in the time intervals observed may appear as linear instead of monoexponential. For this reason, time concentration profiles at $300 \mathrm{~K}$ and $286 \mathrm{~K}$ were examined with a linear regression. The resulting $Z \rightarrow E$ isomerization rates are 10 times $(300 \mathrm{~K})$ or 5 times $(286 \mathrm{~K})$ slower than the rates obtained with the exponential fit. For both linear regressions a smaller Pearson's $\mathrm{R}^{2}$ is obtained compared to the exponential fit. Furthermore, the rate constants obtained by the exponential fit perfectly fit into the Arrhenius plot, whereas using the rate constants obtained by the linear fit worsens Pearson's $\mathrm{R}^{2}$ of the Arrhenius plot significantly. 
Table S9: Half-lives and rate constants of the thermal $Z \rightarrow E$ isomerization of catalyst 6 in DMF- $d_{7}$ at different temperatures.

\begin{tabular}{|c|c|c|c|c|c|}
\hline Temperature [K] & Half-life [h] & $\begin{array}{l}\text { Isomerization rate } \\
\left.\text { (expon. Fit) [mol s }{ }^{-1}\right]\end{array}$ & Pearsons's $\mathbf{R}^{2}$ & $\begin{array}{l}\text { Isomerization rate } \\
\text { (linear fit) }\left[\mathrm{mol} \mathrm{s}^{-1}\right]\end{array}$ & Pearsons's $\mathbf{R}^{2}$ \\
\hline 323 & 1.1 & $6.4 \times 10^{-4}$ & 0.98 & - & - \\
\hline 310 & 4.8 & $1.5 \times 10^{-4}$ & 0.99 & - & - \\
\hline 300 & 15.2 & $4.6 \times 10^{-5}$ & 0.95 & $5 \times 10^{-6}$ & 0.82 \\
\hline 286 & 99.9 & $6.9 \times 10^{-6}$ & 0.79 & $1.167 \times 10^{-6}$ & 0.38 \\
\hline 273 & approx. 1156 & approx. $6.0 \times 10^{-7}$ & 0.13004 & - & - \\
\hline
\end{tabular}

The isomerization rate constant $k$ can be defined according to Arrhenius as:

$$
k=A \cdot e^{-\frac{E_{A}}{R \cdot T}}
$$

The rate constants obtained at the different temperatures are given in table S9. The natural logarithm of Eq. 9 yields the linear equation Eq. 10.

$$
\ln (k)=-\frac{1}{T}+\text { const }
$$

Therefore, the $\ln (k)$ of the measured $k$ values is plotted against the reciprocal temperature. A linear regression of the resulting curve delivers the slope $m$ of the line.

$$
m=-\frac{E_{A}}{R}
$$

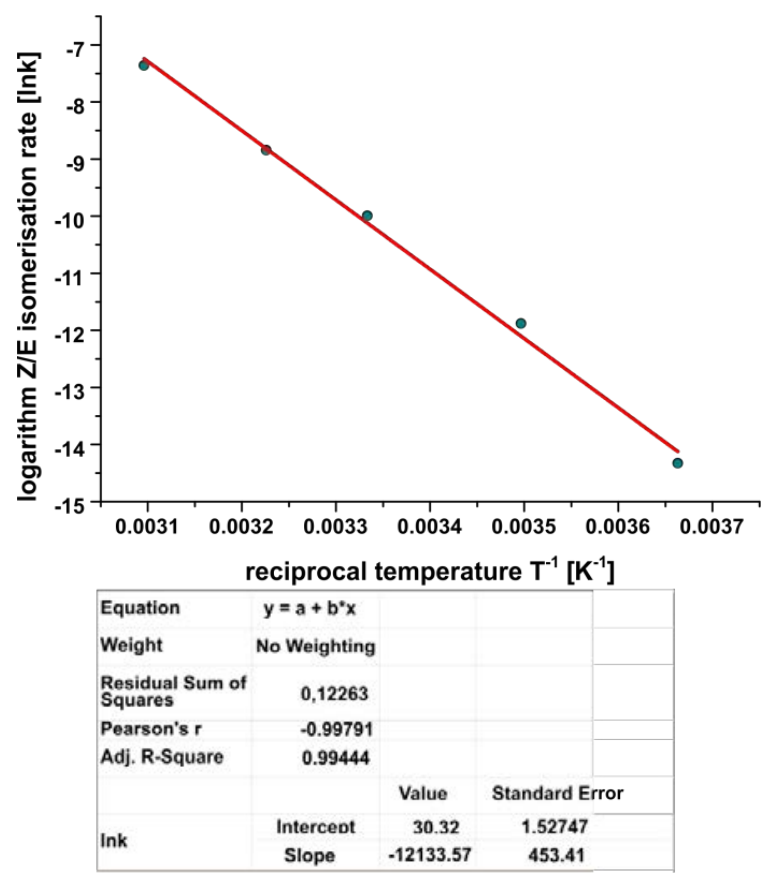

Figure S28: Arrhenius-Plot for the thermal $Z \rightarrow E$ isomerization of catalyst 6 and results of the linear fit procedure.

The resulting activation energy $E_{\mathrm{A}}=-100.9 \mathrm{~kJ} \mathrm{~mol}^{-1}$ or $-24.1 \mathrm{kcal} \mathrm{mol}^{-1}$. The thermal relaxation rate $\mathrm{kZE}_{\mathrm{E}}$ can also be used to calculate the Gibbs energy $\Delta G$ via the Eyring equation.

$$
k_{Z E}=\frac{k_{B} \cdot T}{h} \cdot e^{-\frac{\Delta G}{R \cdot T}}
$$


Table S10: Gibbs energy for the $Z \rightarrow E$ isomerization of compound 6 calculated from rate constants $k$ Z via the Eyring equation

Temperature $[\mathrm{K}]$

323

310

300

286
Isomerization rate $\left[\mathrm{mol} \mathrm{s}^{-1}\right]$

$6.35 \times 10^{-4}$

$1.45 \times 10^{-4}$

$4.57 \times 10^{-5}$

$6.94 \times 10^{-6}$
$\Delta \mathrm{G}\left[\mathrm{kJ} \mathrm{mol} \mathrm{m}^{-1}\right]$

55.81

57.27

58.22

59.87
$\Delta \mathrm{G}\left[\mathrm{kcal} \mathrm{mol}{ }^{-1}\right]$

13.33

13.68

13.91

14.30 


\section{Determination of yields and selectivity via NMR}

The ratio of the respective three different acetylated products was determined via NMR. Therefore, NMR spectra of the crude reaction mixtures were recorded, and the ratio of the products could be directly obtained via integration of the corresponding signals. The yields for 1, 2, 3a and $\mathbf{3 b}$ were determined by comparing the integrals of the benzylic proton of the benzylidene/naphthylidene protecting group with the total integral of the introduced acetyl groups (in case of diacetylated products just the integral of one acetyl moiety was taken into account). The yields of $\mathbf{3} \mathbf{c}$ and $\mathbf{3 d}$ could be directly obtained via integration. Therefore, the integral of the methoxy function was compared with the total integral of the introduced acetyl groups (in case of diacetylated products again just the integral of one acetyl moiety was taken into account). The signals which have been used are marked with triangle, circle, star, and diamond. In case of quercetin 17 yield as well as ratios can be obtained directly via integration and comparison of the assumed aromatic protons. The peak assignments of the corresponding products were done by using 2-D NMR techniques (HSQC, HMBC and COSY) and DEPT135 spectra. 

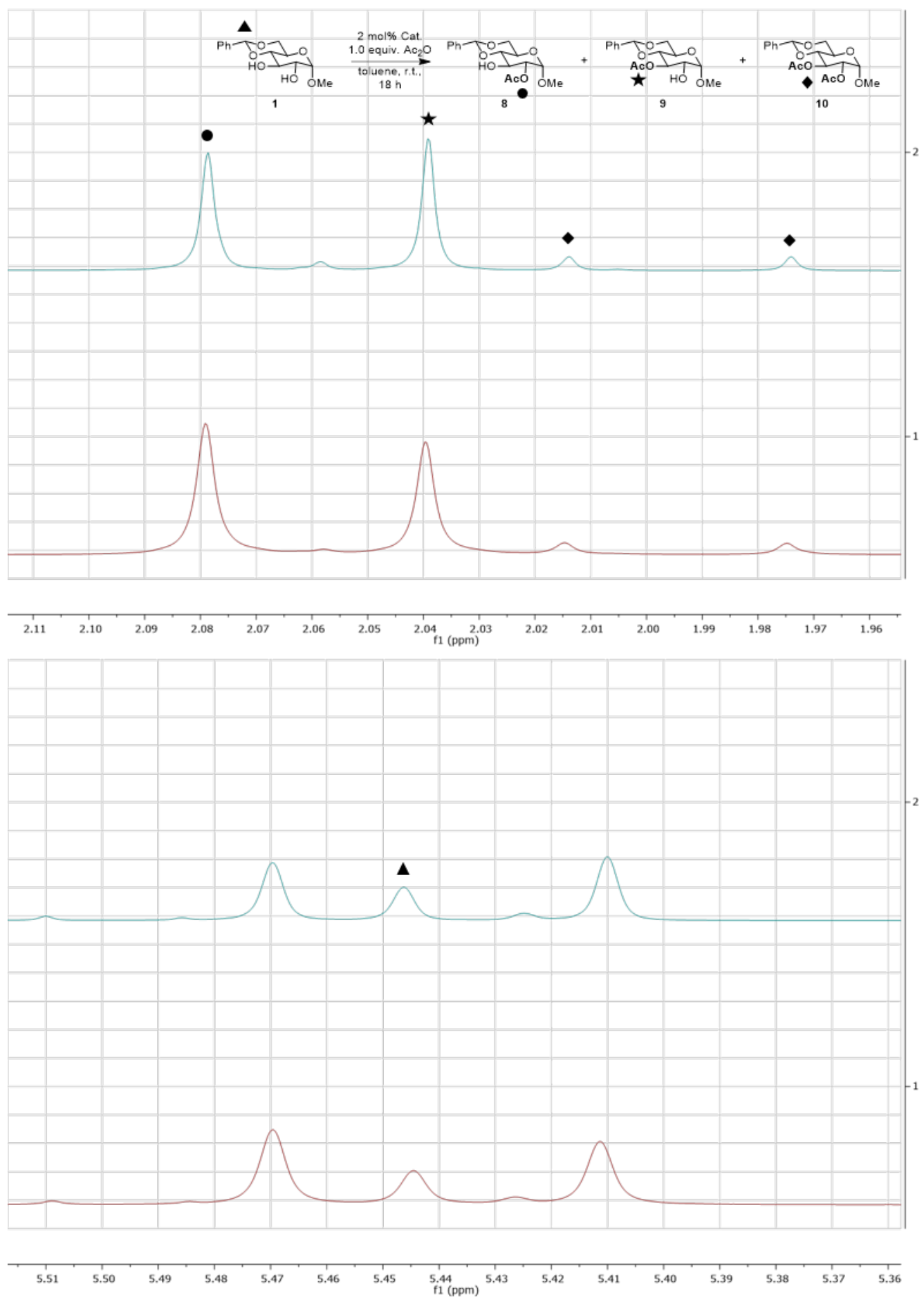

Figure S29 ${ }^{1} \mathrm{H}-\mathrm{NMR}\left(400 \mathrm{MHz}, \mathrm{CDCl}_{3}\right)$ data of the compounds 1, 8-10 resulting from the catalyzed reactions using 6: Reaction under UV irradiation (top) and in the dark (bottom); $1 \delta 5.44(\mathrm{~s}, 1 \mathrm{H}), 8 \delta 2.08(\mathrm{~s}, 3 \mathrm{H}), 9 \delta 2.04(\mathrm{~s}, 3 \mathrm{H})$ and $10 \delta 2.01(\mathrm{~s}, 3 \mathrm{H}), 1.97(\mathrm{~s}, 3 \mathrm{H})$. 

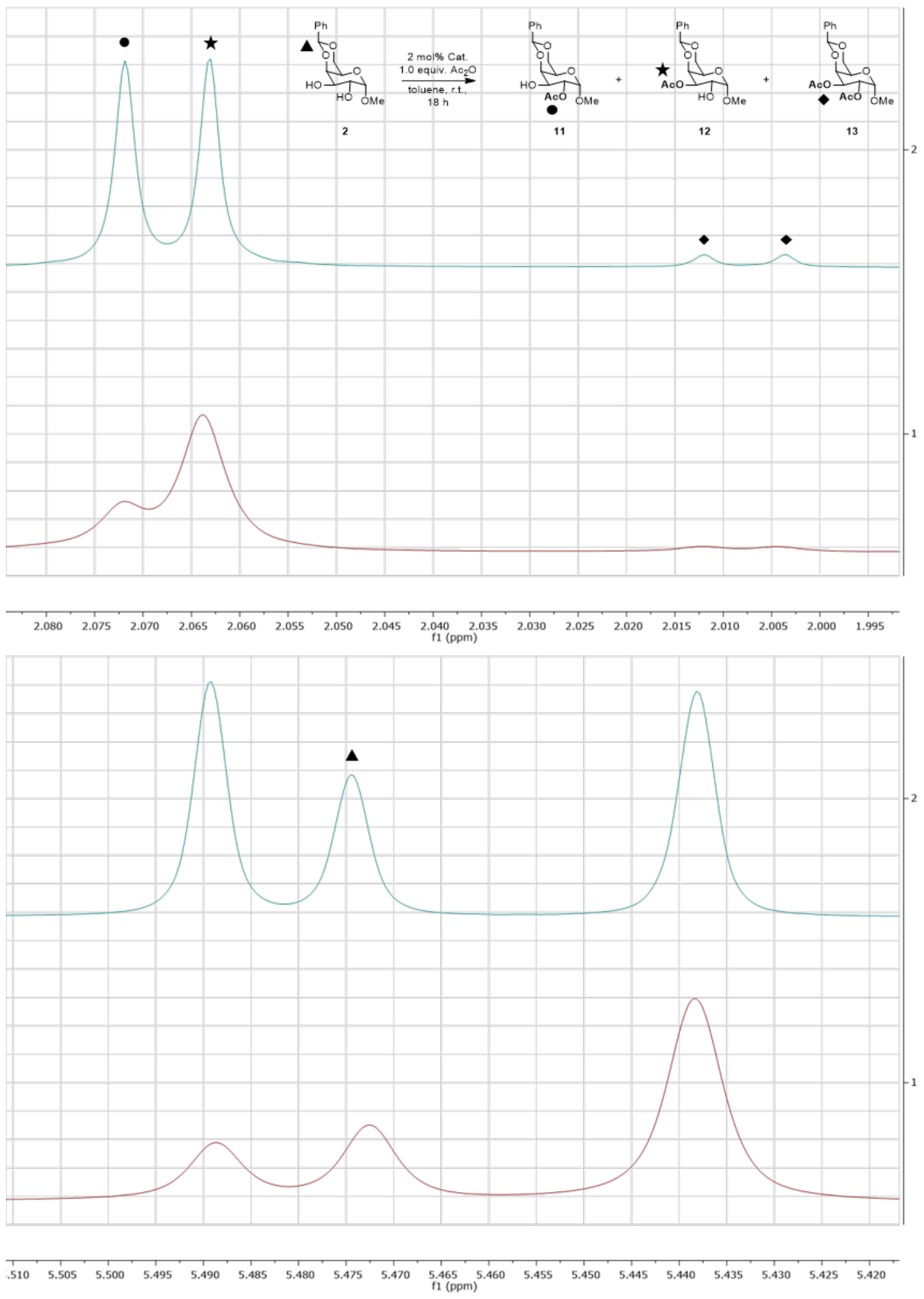

Figure S30 ${ }^{1} \mathrm{H}-\mathrm{NMR}\left(400 \mathrm{MHz}, \mathrm{CDCl}_{3}\right)$ Data of the compounds 2, 11-13 resulting from the catalyzed reactions using 6: Reaction under UV irradiation (top) and in the dark (bottom); $2 \delta 5.47(\mathrm{~s}, 1 \mathrm{H}), 11 \delta 2.07(\mathrm{~s}, 3 \mathrm{H}), 12 \delta 2.06(\mathrm{~s}, 3 \mathrm{H})$ and $13 \delta 2.01(\mathrm{~s}, 3 \mathrm{H}), 2.00(\mathrm{~s}, 3 \mathrm{H})$. 

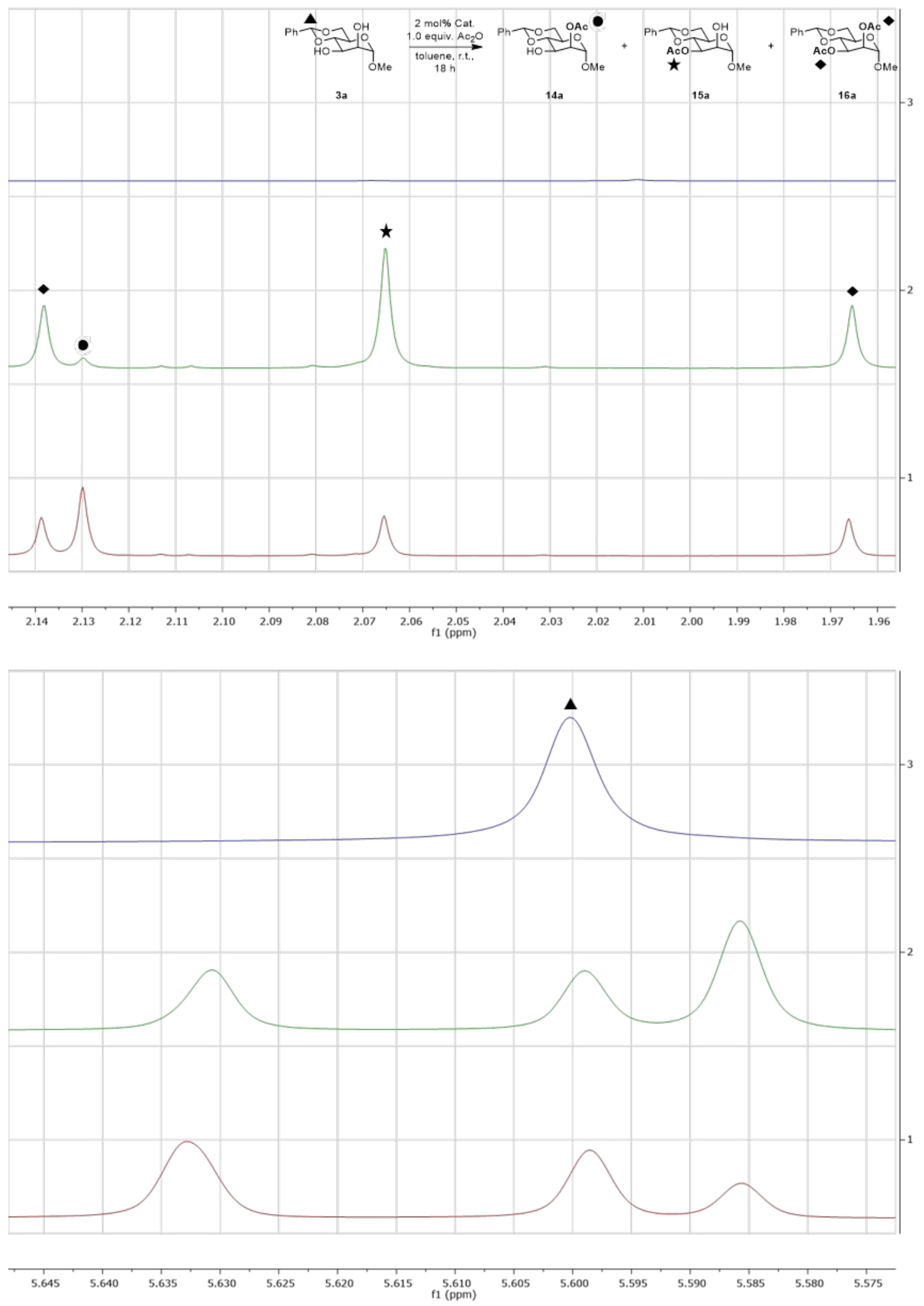

Figure $\mathrm{S} 31{ }^{1} \mathrm{H}-\mathrm{NMR}\left(400 \mathrm{MHz}, \mathrm{MeOH}-d_{4}\right)$ Data of the compounds 3a, 14a-16a resulting from the catalyzed reactions using 6: Starting material (top), reaction under UV irradiation (middle) and in the dark (bottom); 3a $\delta 5.60(\mathrm{~s}, 1 \mathrm{H}), 14 \mathrm{a} \delta 2.13(\mathrm{~s}, 3 \mathrm{H}), 15 \mathrm{a} \delta 2.07(\mathrm{~s}, 3 \mathrm{H})$ and $16 \mathbf{a} \delta 2.14(\mathrm{~s}, 3 \mathrm{H}), 1.97(\mathrm{~s}, 3 \mathrm{H})$. 

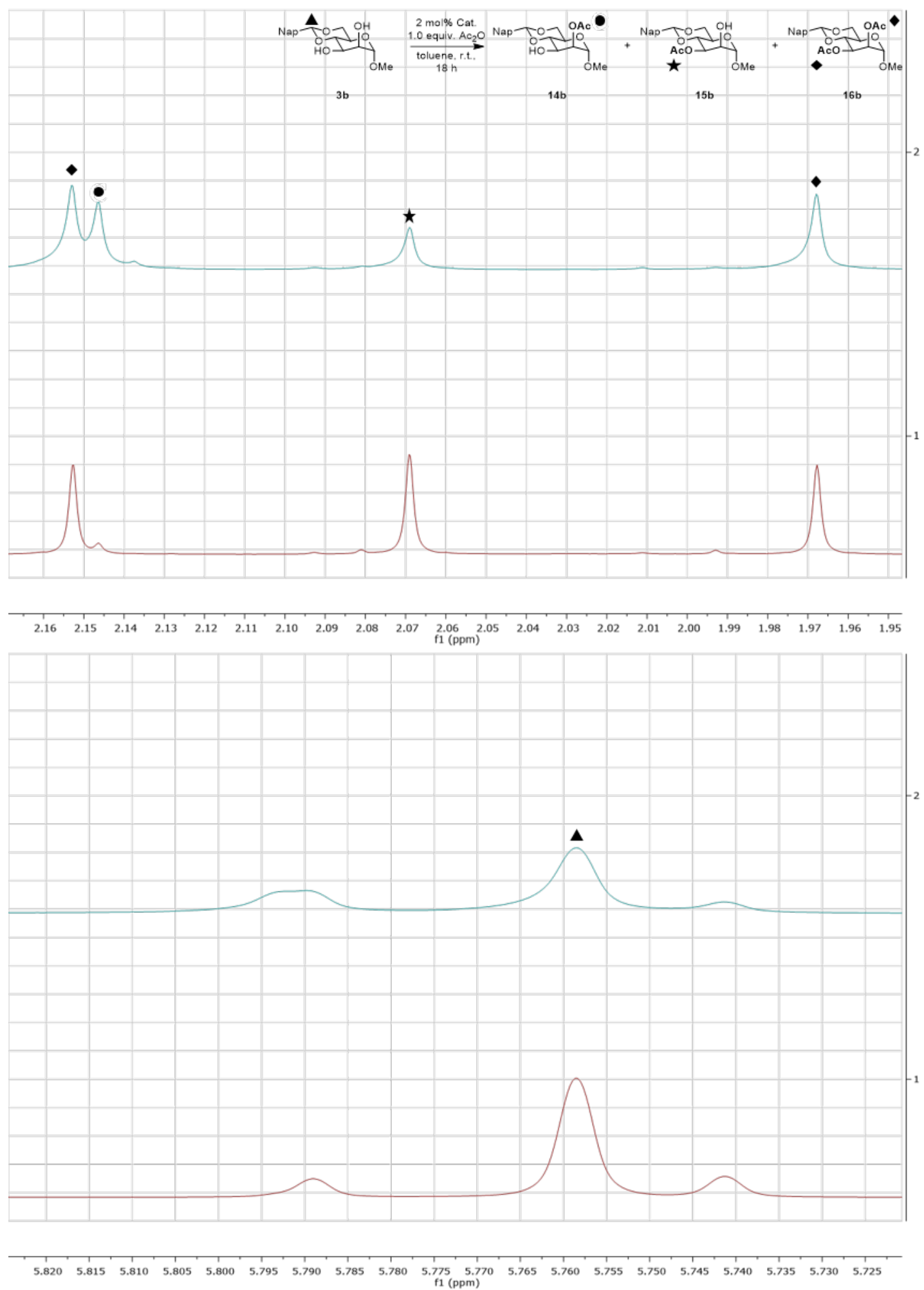

Figure S32 ${ }^{1} \mathrm{H}-\mathrm{NMR}\left(400 \mathrm{MHz}, \mathrm{MeOH}-d_{4}\right)$ Data of the compounds $3 \mathbf{b}, \mathbf{1 4 b}-16 \mathbf{b}$ resulting from the catalyzed reactions using 6 : Reaction under UV irradiation (bottom) and in the dark (top); $3 \mathbf{b} \delta 5.76(\mathrm{~s}, 1 \mathrm{H}), 14 \mathrm{~b} \delta 2.15(\mathrm{~s}, 3 \mathrm{H}), 15 \mathrm{~b} \delta 2.07(\mathrm{~s}, 3 \mathrm{H})$ and $16 \mathrm{~b} \delta 2.16(\mathrm{~s}, 3 \mathrm{H}), 1.97(\mathrm{~s}, 3 \mathrm{H})$. 

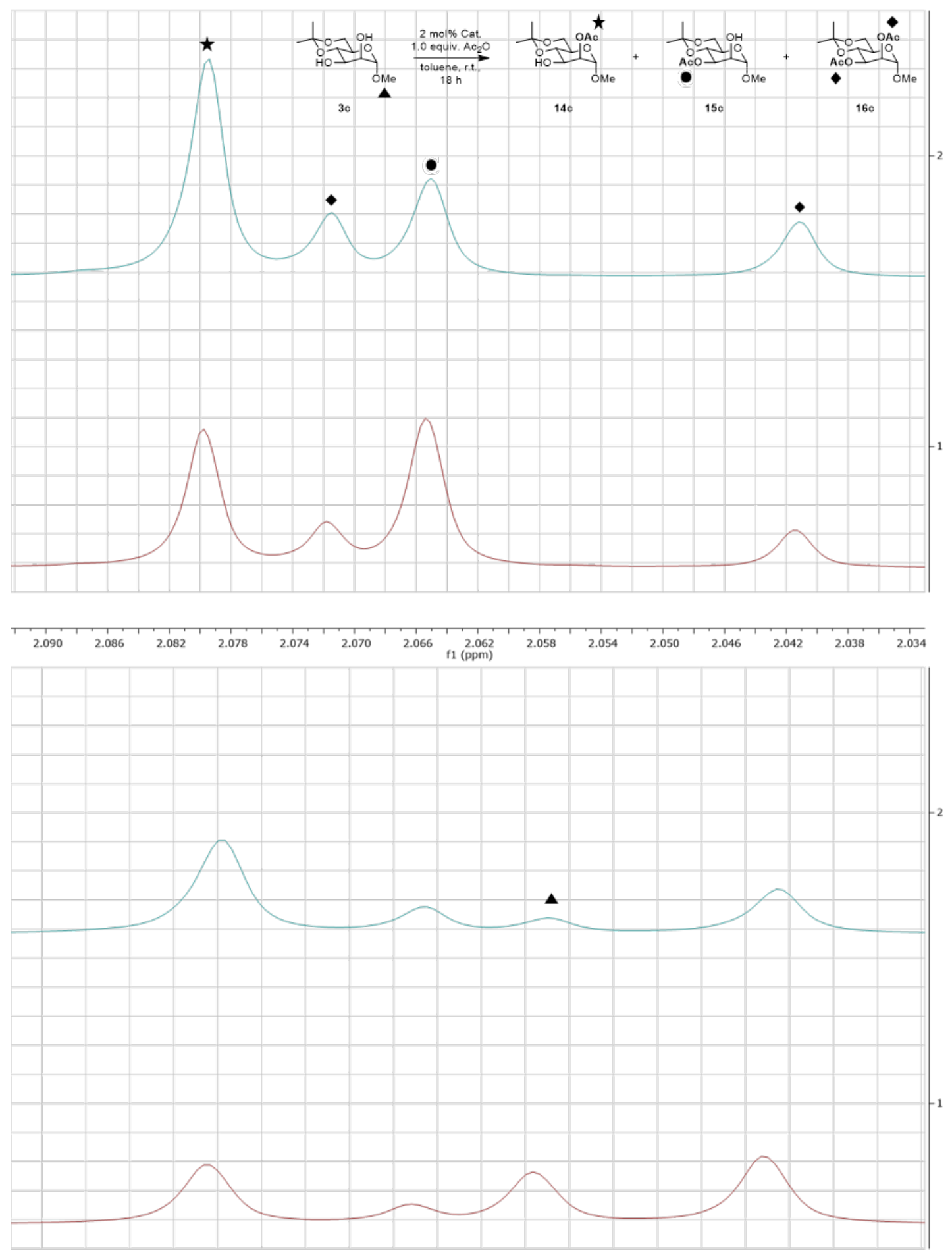

$\begin{array}{llllllllllllllllllllllll}3.420 & 3.418 & 3.416 & 3.414 & 3.412 & 3.410 & 3.408 & 3.406 & 3.404 & 3.402 & 3.400 & 3.398 & 3.396 & 3.394 & 3.392 & 3.390 & 3.388 & 3.386 & 3.384 & 3.382 & 3.380\end{array}$

Figure S33 ${ }^{1} \mathrm{H}-\mathrm{NMR}\left(400 \mathrm{MHz}, \mathrm{MeOH}-\mathrm{d}_{4}\right)$ Data of the compounds $3 \mathrm{c}, 14 \mathrm{c}-16 \mathrm{c}$ resulting from the catalyzed reactions using 6: Reaction under UV irradiation (top) and in the dark (bottom); 3c $\delta 3.40(\mathrm{~s}, 3 \mathrm{H}), 14 \mathrm{c} \delta 2.06(\mathrm{~s}, 3 \mathrm{H}), 15 \mathrm{c} \delta 2.08(\mathrm{~s}, 3 \mathrm{H})$ and $16 \mathrm{c} \delta 2.07(\mathrm{~s}, 3 \mathrm{H}), 2.04(\mathrm{~s}, 3 \mathrm{H})$. 

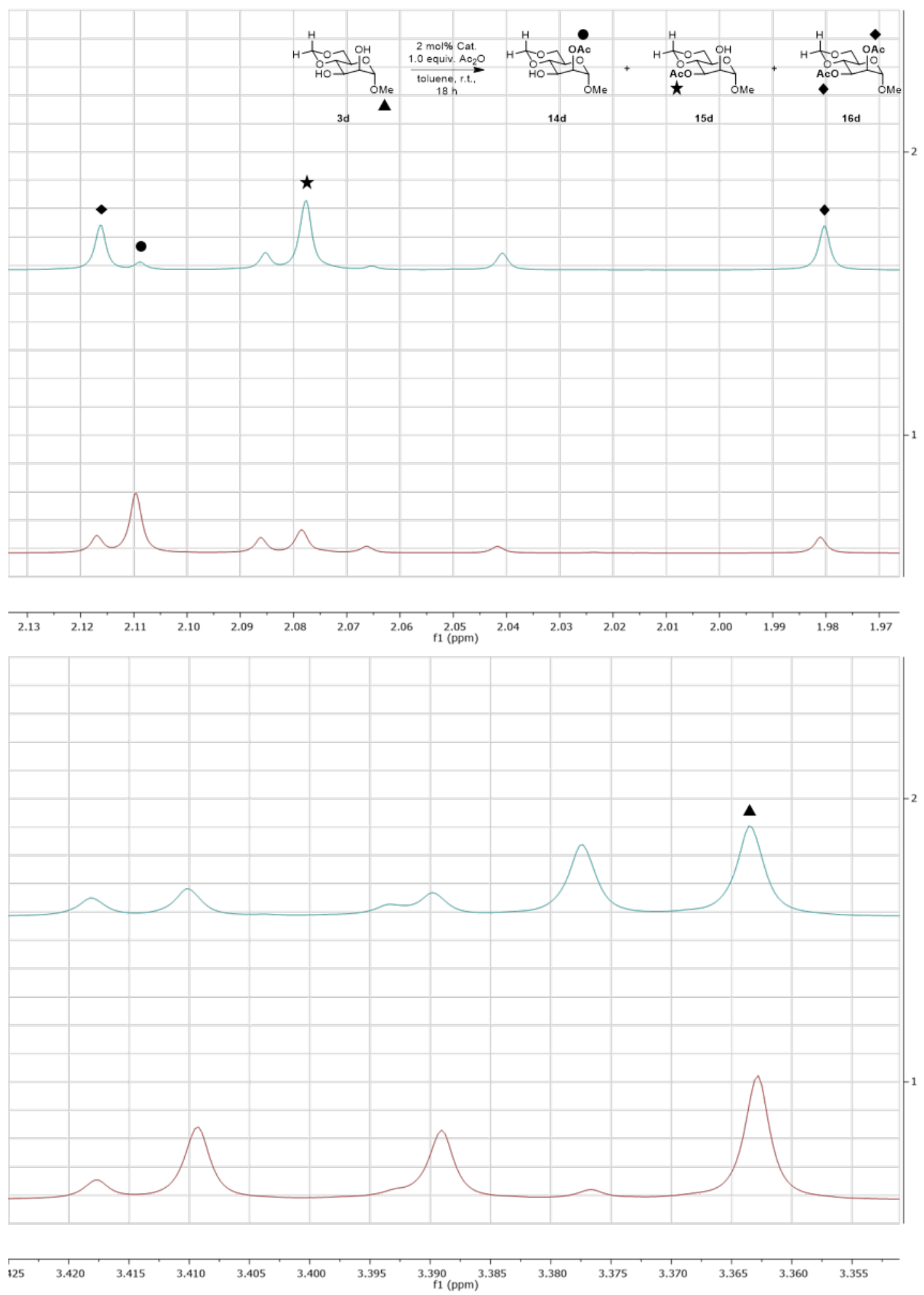

Figure S34 ${ }^{1} \mathrm{H}-\mathrm{NMR}\left(400 \mathrm{MHz}, \mathrm{MeOH}-\mathrm{d}_{4}\right)$ Data of the compounds 3d, 14d-16d resulting from the catalyzed reactions using 6: Reaction under UV irradiation (top) and in the dark (bottom); 3d $\delta 3.36(\mathrm{~s}, 3 \mathrm{H}), 14 \mathrm{~d} \delta 2.11(\mathrm{~s}, 3 \mathrm{H}), 15 \mathrm{~d} \delta 2.08(\mathrm{~s}, 3 \mathrm{H})$ and $16 \mathrm{~d} \delta 2.12(\mathrm{~s}, 3 \mathrm{H}), 1.98(\mathrm{~s}, 3 \mathrm{H})$. 

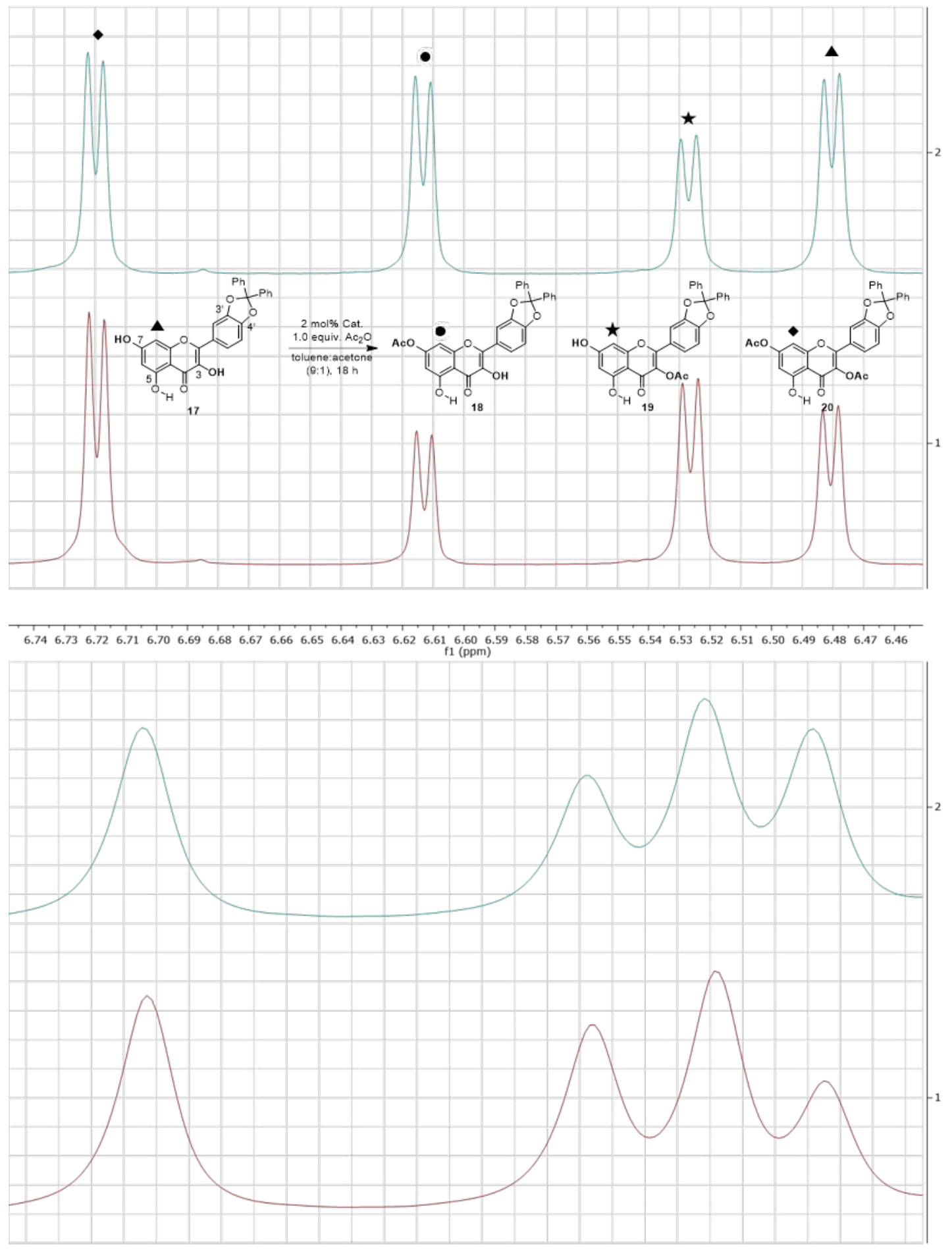

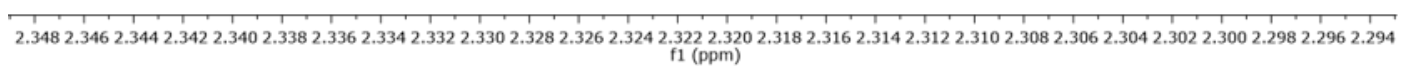

Figure S35. ${ }^{1} \mathrm{H}-\mathrm{NMR}\left(400 \mathrm{MHz}\right.$, DMSO- $\left.d_{6}\right)$ Data of the compounds 17-20 resulting from the catalyzed reactions using 6: reaction under UV irradiation (top) and in the dark (bottom); $20 \delta 2.30(\mathrm{~s}, 3 \mathrm{H}), 2.34(\mathrm{~s}, 3 \mathrm{H}), 6.72(\mathrm{~d}, J=2.0 \mathrm{~Hz}, 1 \mathrm{H}), 18 \delta 2.30(\mathrm{~s}, 3 \mathrm{H}), 6.61(\mathrm{~d}, J=2.0 \mathrm{~Hz}, 1 \mathrm{H}), 19 \delta 2.31(\mathrm{~s}, 3 \mathrm{H}), 6.53(\mathrm{~d}, J=2.0 \mathrm{~Hz}, 1 \mathrm{H})$ and $17 \delta 6.48(\mathrm{~d}, \mathrm{~J}=2.0 \mathrm{~Hz}, 1 \mathrm{H})$.

S51 


\section{Additional Results}

Table S11. Acetylation of (4,6-O-benzylidene)methyl- $\alpha$-D-glucopyranoside (1).

\begin{tabular}{|c|c|c|c|c|c|c|}
\hline & 1 & $\begin{array}{c}2 \mathrm{~mol} \% \mathrm{Cat} . \\
\begin{array}{c}1.0 \text { equiv. } \mathrm{Ac}_{2} \mathrm{O} \\
\text { toluene, r.t., } \\
18 \mathrm{~h}\end{array}\end{array}$ & 8 & 9 & AcO OMe & \\
\hline Entry & Catalyst & & $8 / \%$ & $9 / \%$ & $10 / \%$ & Conversion / \% \\
\hline 1 & NMI & & 18 & 61 & 5 & 84 \\
\hline 2 & 4 & & 46 & 28 & 7 & 81 \\
\hline 3 & $4(365 \mathrm{~nm})$ & & 51 & 28 & 11 & 90 \\
\hline 4 & 5 & & 43 & 17 & 2 & 62 \\
\hline 5 & $5(365 \mathrm{~nm})$ & & 46 & 29 & 8 & 83 \\
\hline 6 & 6 & & 42 & 35 & 4 & 81 \\
\hline 7 & $6(365 \mathrm{~nm})$ & & 37 & 39 & 4 & 80 \\
\hline
\end{tabular}

Yields determined via ${ }^{1} \mathrm{H}$ NMR (Fig. S29). NMI = N-methylimidazole.

Table S12. Acetylation of (4,6-O-benzylidene)methyl- $\alpha$-D-galactopyranoside (2).

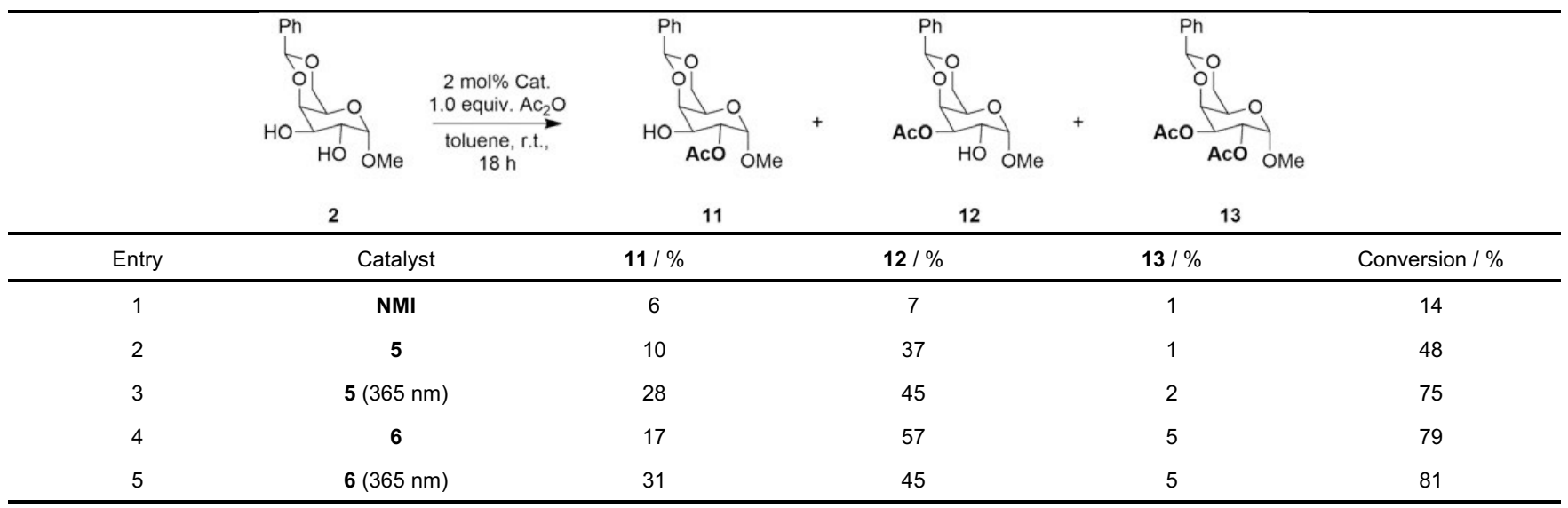

Yields determined via ${ }^{1} \mathrm{H}$ NMR (Fig. S30). NMI = N-methylimidazole

Table S13. Acetylation of (4,6-O-benzylidene)methyl- $\alpha$-D-mannopyranoside (3a).

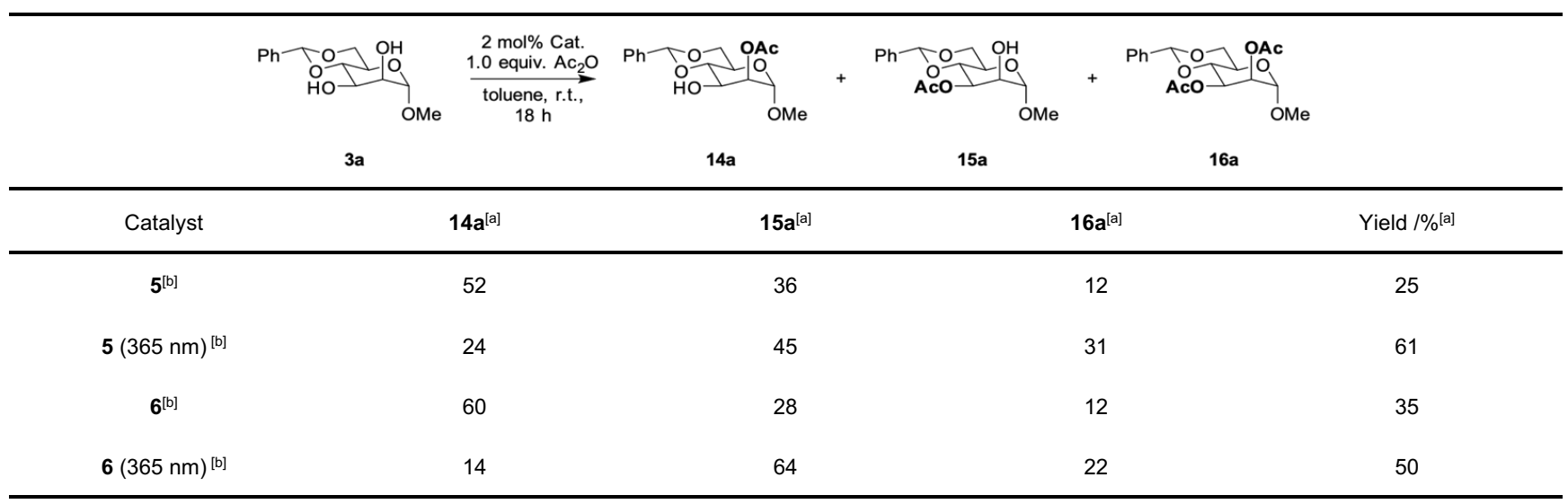

[a] Yield and ratio determined via ${ }^{1} \mathrm{H}$ NMR. [b] $4 \mathrm{~h}$ reaction time. Yields determined via ${ }^{1} \mathrm{H}$ NMR (Fig. S31). 


\section{References}

(1) Frisch, M. J.; Trucks, G. W.; Schlegel, H. B.; Scuseria, G. E.; Robb, M. A.; Cheeseman, J. R.; Scalmani, G.; Barone, V.; Petersson, G. A.; Nakatsuji, H.; Li, X.; Caricato, M.; Marenich, A. V.; Bloino, J.; Janesko, B. G.; Gomperts, R.; Mennucci, B.; Hratchian, H. P.; Ortiz, J. V.; Izmaylov, A. F.; Sonnenberg, J. L.; Williams; Ding, F.; Lipparini, F.; Egidi, F.; Goings, J.; Peng, B.; Petrone, A.; Henderson, T.; Ranasinghe, D.; Zakrzewski, V. G.; Gao, J.; Rega, N.; Zheng, G.; Liang, W.; Hada, M.; Ehara, M.; Toyota, K.; Fukuda, R.; Hasegawa, J.; Ishida, M.; Nakajima, T.; Honda, Y.; Kitao, O.; Nakai, H.; Vreven, T.; Throssell, K.; Montgomery Jr., J. A.; Peralta, J. E.; Ogliaro, F.; Bearpark, M. J.; Heyd, J. J.; Brothers, E. N.; Kudin, K. N.; Staroverov, V. N.; Keith, T. A.; Kobayashi, R.; Normand, J.; Raghavachari, K.; Rendell, A. P.; Burant, J. C.; Iyengar, S. S.; Tomasi, J.; Cossi, M.; Millam, J. M.; Klene, M.; Adamo, C.; Cammi, R.; Ochterski, J. W.; Martin, R. L.; Morokuma, K.; Farkas, O.; Foresman, J. B.; Fox, D. J. Wallingford, CT, 2016.

(2) Grimme, S.; Antony, J.; Ehrlich, S.; Krieg, H. A consistent and accurate ab initio parametrization of density functional dispersion correction (DFT-D) for the 94 elements H-Pu. J. Chem. Phys. 2010, 132, 154104

(3) Schweighauser, L.; Strauss, M. A.; Bellotto, S.; Wegner, H. A. Attraction or Repulsion? London Dispersion Forces Control Azobenzene Switches. Angew. Chem. Int. Ed. 2015, 54, 13436-13439.

(4) (a)Frank, N. The ORCA program system. WIREs Comput Mol Sci 2012, 2, 73-78; $\quad$ (b)Riplinger, C.; Sandhoefer, B.; Hansen, A.; Neese, F. Natural triple excitations in local coupled cluster calculations with pair natural orbitals. J. Chem. Phys. 2013, 139, 134101.

(5) (a)Cattaneo, P.; Persico, M. An abinitio study of the photochemistry of azobenzene. PCCP 1999, 1, 4739-4743; (b)Cembran, A.; Bernardi, F.; Garavelli, M.; Gagliardi, L.; Orlandi, G. On the Mechanism of the cis-trans Isomerization in the Lowest Electronic States of Azobenzene: S0, S1, and T1. J. Am. Chem. Soc 2004, 126, 3234-3243; (c)Dokić, J.; Gothe, M. Wirth J.: Peters, M. V. Schwarz, J.; Hecht, S.; Saalfrank, P. Quantum Chemical Investigation of Thermal Cis-toTrans Isomerization of Azobenzene Derivatives: Substituent Effects, Solvent Effects, and Comparison to Experimental Data. J. Phys. Chem. A 2009, 113, 6763-

6773; (d)Bandara, H. M. D.; Burdette, S. C. Photoisomerization in different classes of azobenzene. Chem. Soc. Rev. 2012, 41, $1809-1825$

(6) Haessner, C.; Mustroph, H. Untersuchungen zum UV/VIS-Spektralverhalten von Azofarbstoffen. XV. Eine Analyse der Absorptionsspektren von 4,4'Diaminoazobenzenen. J. prakt. Chem. 1986, 328, 113-119.

(7) Brode, W. R.; Gould, J. H.; Wyman, G. M. The Relation between the Absorption Spectra and the Chemical Constitution of Dyes. XXV. Phototropism and cis-trans Isomerism in Aromatic Azo Compounds1. J. Am. Chem. Soc. 1952, 74, 4641-4646.

(8) (a)Feldmeier, C.; Bartling, H.; Riedle, E.; Gschwind, R. M. LED based NMR illumination device for mechanistic studies on photochemical reactions Versatile and simple, yet surprisingly powerful. J. Magn. Reson. 2013, 232, 39-44; (b)Wolff, C.; Kind, J.; Schenderlein, H.; Bartling, H.; Feldmeier, C.; Gschwind, R. M.; Biesalski, M.; Thiele, C. M. Studies of a photochromic model system using NMR with ex-situ and in-situ irradiation devices. Magn. Reson. Chem. 2016, 54, 485-491; (c)Kind, J.; Kaltschnee, L.; Leyendecker, M.; Thiele, C. M. Distinction of trans-cis photoisomers with comparable optical properties in multiple-state photochromic systems - examining a molecule with three azobenzenes via in situ irradiation NMR spectroscopy. Chem. Commun. 2016, 52, 12506-12509.

(9) Procházková, E.; Kolmer, A.; llgen, J.; Schwab, M.; Kaltschnee, L.; Fredersdorf, M.; Schmidts, V.; Wende, R. C.; Schreiner, P. R.; Thiele, C. M. Uncovering Key Structural Features of an Enantioselective Peptide-Catalyzed Acylation Utilizing Advanced NMR Techniques. Angew. Chem. Int. Ed. 2016, 55, 15754-15759. 